\title{
Gravitational Lensing and the MaXimum Number of Images
}

by

Johann Bayer

A thesis submitted in conformity with the requirements

for the degree of Doctor of Philosophy

Graduate Department of Physics

University of Toronto

Copyright (c) 2008 by Johann Bayer 


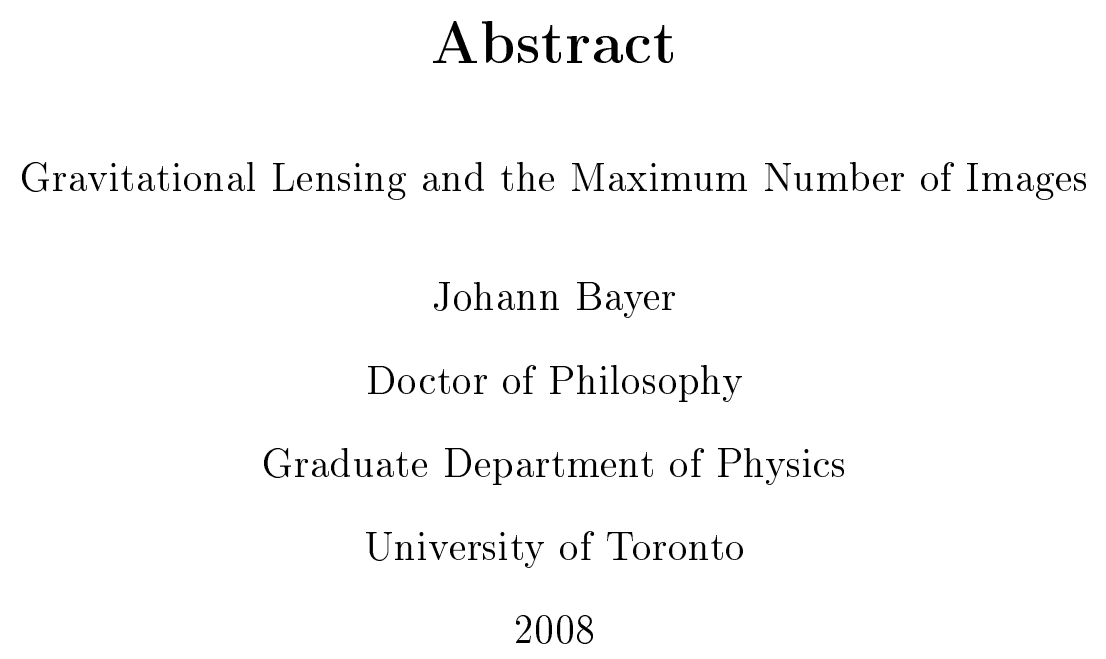

Gravitational lensing, initially a phenomenon used as a solid confirmation of General Relativity, has defined itself in the past decade as a standard astrophysical tool. The ability of a lensing system to produce multiple images of a luminous source is one of the aspects of gravitational lensing that is exploited both theoretically and observationally to improve our understanding of the Universe.

In this thesis, within the field of multiple imaging we explore the case of maximal lensing, that is, the configurations and conditions under which a set of deflecting masses can produce the maximum number of images of a distant luminous source, as well as a study of the value for this maximum number itself.

We study the case of a symmetric distribution of $n-1$ point-mass lenses at the vertices of a regular polygon of $n-1$ sides. By the addition of a perturbation in the form of an $n^{t h}$ mass at the center of the polygon it is proven that, as long as the mass is small enough, the system is a maximal lensing configuration that produces $5(n-1)$ images. Using the explicit value for the upper bound on the central mass that leads to maximal lensing, we illustrate how this result can be used to find and constrain the mass of planets or brown dwarfs in multiple star systems.

For the case of more realistic mass distributions, we prove that when a point-mass is replaced with a distributed lens that does not overlap with existing images or lensing objects, an additional image is formed within the distributed mass while positions and 
numbers of existing images are left unchanged. This is then used to conclude that the maximum number of images that $n$ isolated distributed lenses can produce is $6(n-1)+1$.

In order to explore the likelihood of observational verification, we analyze the stability properties of the symmetric maximal lensing configurations. Finally, for the cases of $n=4,5$ and 6 point-mass lenses, we study asymmetric maximal lensing configurations and compare their stability properties against the symmetric case. 


\section{Dedication}

To my parents,

for their patience and love,

for the discipline they taught me,

and for the passion for knowledge

with which they gifted me. 


\section{Acknowledgements}

More than anybody else, I would like to thank my research supervisor, Professor Charles C. Dyer. Without his advice, support and guidance none of this would have been possible. He kept me focused on the path when the goal was the hardest to find while at the same time providing me with the freedom and encouragement to explore and discover my passion for teaching.

I would also like to thank Professor Michael Luke and Professor Slavek Rucinski, members of my research supervising committee, for their valuable comments and insight into my research.

My thanks to my friend and colleague Brian Wilson, with whom discussions about science, learning and life gave me always new viewpoints and helped me through the experience of studying, living and doing research in a foreign country.

To Dan Giang and John Liska, my thanks for their collaboration during their M.Sc. studies exploring the numerical and approximate details of multiple imaging.

I am very grateful to my colleague Allen Attard for his patience and support in dealing with everything related with computers. Without his exceptional computer knowledge, this research would have encountered significant obstacles.

I thank my parents and siblings for all their support and encouragement that made themselves be felt throughout the distance. And to my partner Sherry for her being there to keep me sane while writing this document.

This research was funded in large part by the Natural Sciences and Engineering Research Council (Discovery Grant) and the University of Toronto. 


\section{Contents}

1 Introduction $\quad 1$

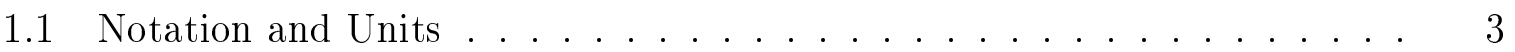

1.2 Light Propagation and Optical Scalars . . . . . . . . . . . . 5

1.3 Cosmology and Distances ..................... 11

1.4 Light Bending and the Lens Equation . . . . . . . . . . . . . 17

1.5 Properties of the Lens Equation . . . . . . . . . . . . . 24

2 Image Counting $\quad 27$

2.1 Multiple Imaging . . . . . . . . . . . . . . . . . . 28

2.2 The Odd Number Theorem . . . . . . . . . . . . . . 34

2.3 Bounds on the Number of Images . . . . . . . . . . . . . 36

2.4 A Theorem in Complex Analysis . . . . . . . . . . . . . . 41

3 Symmetric Point-Mass Configurations 43

3.1 Central Mass for Maximal Lensing . . . . . . . . . . . . . 44

3.2 Properties of $m_{\star}$ as an Upper Bound . . . . . . . . . . 52

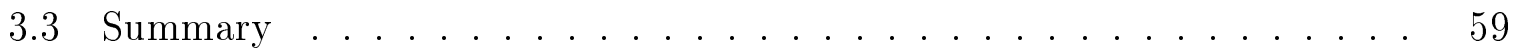

4 Lensing and Distributed Lenses $\quad 60$

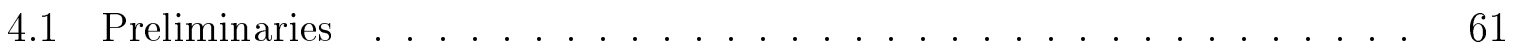

4.2 From Point Masses to Distributed Lenses . . . . . . . . . . . . . 62 


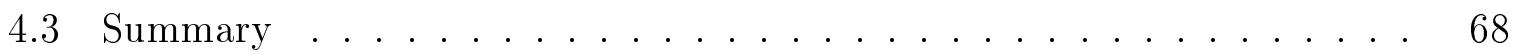

5 Stability and Applications $\quad 69$

5.1 Perturbations on Symmetric Configurations . . . . . . . . . . 69

5.2 Applications of the Symmetric Configurations . . . . . . . . . . 76

5.3 Asymmetric Configurations . . . . . . . . . . . . . . 81

5.4 Perturbations on Asymmetric Configurations . . . . . . . . . . 88

6 Conclusions $\quad 94$

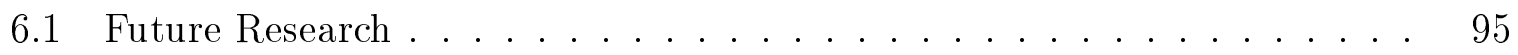

$\begin{array}{ll}\text { Bibliography } & 97\end{array}$

A Some Mathematical Proofs 105

A.1 Minimum Value for the Critical Radius . . . . . . . . . . . . 105

A.2 Monotonicity of the Bound for the Central Mass . . . . . . . . . . . 108

B Numerical Simulations $\quad 112$

B.1 Mass Finding Code . . . . . . . . . . . . . . 113

B.2 Perturbations on Symmetric Configurations . . . . . . . . . . . 117

B.3 Searching for Asymmetric Configurations . . . . . . . . . . . . . . 121

B.4 Perturbations on Asymmetric Configurations . . . . . . . . . . . . 124 


\section{List of Figures}

1.1 Point-mass gravitational lens configuration (Schwarzschild lens) . . . . . 19

1.2 Generic single-plane gravitational lens configuration . . . . . . . . . . 23

3.1 Symmetric point-mass lensing for 5 lenses in a regular pentagon . . . . . 45

3.2 Critical radius of the symmetric polygon configuration as a function of the

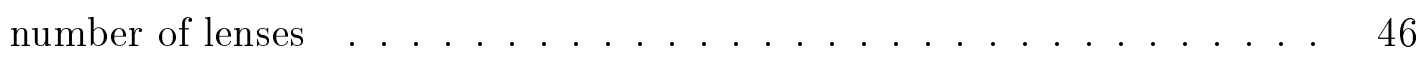

3.3 Central mass perturbation on a configuration of 5 point-mass lenses in a

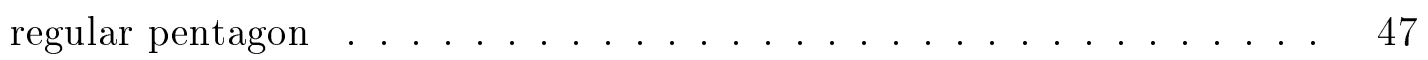

3.4 Graphs of $f_{+}(\rho)$ as a function of the number of lenses . . . . . . . 49

3.5 Graphs of $f_{+}(\rho)$ as a function of the number of lenses (Detail) . . . . 50

3.6 Graphs of $f_{-}(\rho)$ as a function of the number of lenses . . . . . . 51

3.7 Graphs of $f_{-}(\rho)$ as a function of the number of lenses (Detail) . . . . 52

3.8 Critical mass for maximal lensing as a function of the number of lenses and the radius of the polygon $\ldots \ldots \ldots \ldots$

3.9 Central mass perturbation of $m<m_{\star}$ on a configuration of point masses

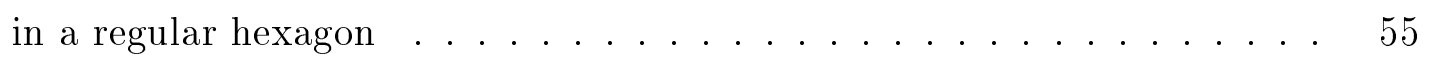

3.10 Central mass perturbation of $m>m_{\star}$ on a configuration of point masses

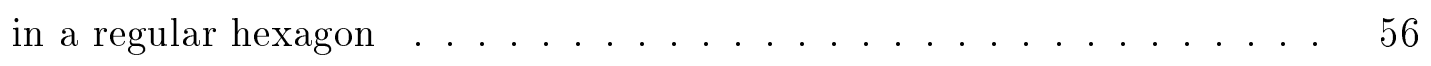

3.11 Maximum mass for maximal lensing for $r=\frac{1}{2}$ as a function of the number

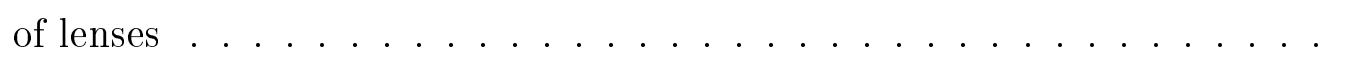


3.12 Maximum mass for maximal lensing for $r=\frac{1}{10}$ as a function of the number

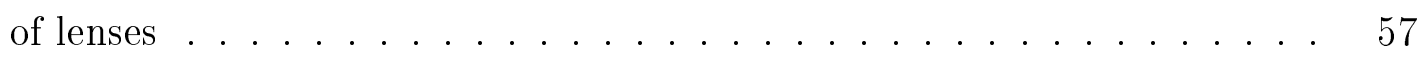

3.13 Maximum mass for maximal lensing for $r=\frac{1}{4}$ as a function of the number

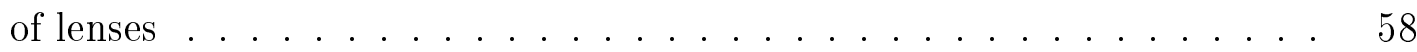

3.14 Maximum mass for maximal lensing for $r=\frac{7}{10}$ as a function of the number

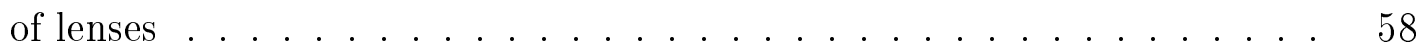

5.1 Stability of the maximal lensing configuration under perturbations to the position of a lens in the polygon . . . . . . . . . . . . . 71

5.2 Stability of the maximal lensing configuration under perturbations to the position of the central mass . . . . . . . . . . . . 73

5.3 Stability of the maximal lensing configuration under perturbations to the position of the source . . . . . . . . . . . . . 74

5.4 Stability of the maximal lensing configuration under perturbations to the

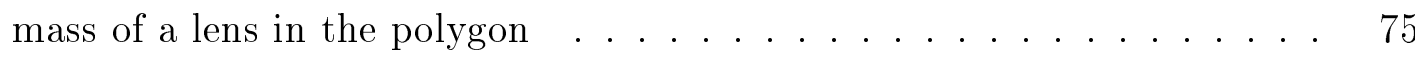

5.5 Maximal lensing configuration of 3 point-mass lenses in an equilateral triangle with a small central lens . . . . . . . . . . . . 77

5.6 Maximal lensing configuration of 3 point-mass lenses in an equilateral triangle with a small central lens (Detail) . . . . . . . . . 78

5.7 Microlensing light-curve for a small mass in transit through the center of a maximal lensing configuration . . . . . . . . . . . 82

5.8 Microlensing light-curve for a small mass in transit through the center of a maximal lensing configuration (Detail) . . . . . . . . 82

5.9 Microlensing light-curve for a small mass in transit off the center of a symmetric configuration ..................... 83

5.10 Microlensing light-curve for a small mass in transit off the center of a symmetric configuration (Detail) . . . . . . . . . 83

5.11 Asymmetric maximal lensing for 4 point-mass lenses . . . . . . . . . 85 
5.12 Asymmetric maximal lensing for 5 point-mass lenses . . . . . . . . . . 87

5.13 Asymmetric lensing for 6 point-mass lenses with 21 images . . . . . . . 89 


\section{Chapter 1}

\section{Introduction}

The world, as we currently understand it, is described in physical terms by two highly successful theories: Quantum Mechanics and General Relativity. Both these centenarian theories, despite numerous experimental confirmations and the vast array of confirmed predictions, remain interestingly incompatible and thus suggest that all is not yet discovered and that the Universe will not disappoint our eagerness and capacity for amazement.

In both these theories there remain phenomena that have been known for decades and, thankfully for those of us that like doing physics, still have details that need to be investigated and understood.

General Relativity, after successfully explaining the residual precession of Mercury's perihelion (Einstein, 1915), had in the bending of light rays due to the presence of a gravitational field (Einstein, 1916) its most important prediction, test and confirmation. Observed first during the solar eclipse of 1919 (Eddington, 1920), the deflection of light rays due to gravitational fields grew to become the field of study known as gravitational lensing.

Gravitational lensing has since turned into a fundamental astrophysical tool (Wambsganss, 2000, 2006) and is currently used in fields as varied as dark matter and dark energy

probing, large scale structure studies, determination of cosmological parameters, extra- 
solar planetary search, mass profile reconstruction and source morphology reconstruction, amongst others.

One of the most intriguing aspects of gravitational lensing is the possibility for a distant luminous source to produce multiple images when a localized intervening matter distribution acts as a lens between the source and the observer. However, almost ninety years after the first observation of light deflection (Eddington, 1920) and almost thirty years after the discovery of the first multiple imaged system (Walsh et al., 1979; Walsh, 1989), gaps remain in our understanding of the production of multiple images.

Maximal lensing, the study of gravitational lensing configurations that produce the maximum number of images, is one area where current understanding of the lensing phenomena still has open questions and is the focus of this thesis. We will address the maximal lensing problem through the question: What is the maximum number of images that a set of $n$ gravitational lenses can produce of a distant luminous source?

In this chapter we introduce the basic theory of gravitational lensing as it is needed to establish the lens equation. The lens equation represents a transformation that describes a large number of lensing situations both in theory and from observations. Therefore, maximal lensing results obtained by studying the lens equation will be generic.

In Chapter 2, we present a review of the existing literature in multiple imaging, image counting and the establishing of bounds to the maximum number of images a configuration of lenses can produce.

In Chapter 3, we derive the main result for the maximum number of images for a set of $n$ point-mass lenses on a plane. This result is an extension of work previously published in Bayer and Dyer (2007).

In Chapter 4, the result for point-mass lenses is extended to distributed masses. This chapter is an extension of work previously published in Bayer et al. (2006).

In Chapter 5, we study the stability properties of the results found for point-mass lenses. Furthermore, we explore a possible application of maximal lensing to the search 
for planets and brown dwarfs in multiple star systems.

Presented in Chapter 6 is a summary and synthesis of the main results of this thesis and provides a list of research to be done in the area.

We have added in Appendix B the Mathematica codes used in the simulations for the analysis of stability as well as for searching for maximal lensing configurations.

\subsection{Notation and Units}

The speed of light in vacuum is $c=3.00 \times 10^{8} \mathrm{~m} / \mathrm{s}$ and the gravitational constant is $G=6.67 \times 10^{-11} \mathrm{~N} \cdot \mathrm{m}^{2} / \mathrm{kg}^{2}$.

A few astronomical units will be used as follows. The length of the semi-major axis of the orbit of the Earth around the Sun is the astronomical unit, given by 1 au $=$ $1.50 \times 10^{11} \mathrm{~m}$ and the distance light travels in one year is the light-year, given by $1 \mathrm{ly}=$ $9.46 \times 10^{15} \mathrm{~m}$. For larger distances, it will be convenient to use the parsec and kiloparsec, given by $1 \mathrm{pc}=3.26 \mathrm{ly}$ and $1 \mathrm{kpc}=3.26 \times 10^{3}$ ly respectively.

The mass of the Sun is the solar mass given by $\mathrm{M}_{\odot}=1.99 \times 10^{30} \mathrm{~kg}$, and the mass of Jupiter is $\mathrm{M}_{\mathrm{J}}=1.90 \times 10^{27} \mathrm{~kg}$.

The symbol $i$ represents the imaginary unit such that $i^{2}=-1$, the set of all complex numbers is $\mathbb{C}$ and for $z \in \mathbb{C}$, the complex conjugate and magnitude of $z$ are $\bar{z}$ and $|z|$ respectively. Similarly, for $z \in \mathbb{C}$, the real and imaginary parts of $z$ are $\operatorname{Re}(z)$ and $\operatorname{Im}(z)$ respectively. The degree of polynomial $p(w)$ is noted as $\operatorname{deg} p$ or $\operatorname{deg} p(w)$.

Vector quantities are represented by bold typeface and the symbols $\nabla$ and $\triangle$ denote the two dimensional gradient and Laplacian respectively.

Standard tensor notation is used with covariant and contravariant indices as subscripts and superscripts respectively. Einstein's summation convention over repeated indices is used for vectors and tensors. Roman indices indicate all space-time coordinates numbered $(0,1,2,3)$ with 0 as the time-like component. Greek indices label the three 
spatial coordinates numbered $(1,2,3)$. Thus, for example

$$
x^{a}=\left(x^{0}, x^{1}, x^{2}, x^{3}\right) \text { and } \quad x^{\alpha}=\left(x^{1}, x^{2}, x^{3}\right) .
$$

The signature of the space-time metric $g_{a b}$ is taken to be -2, making the orthonormalized Minkowski metric $\eta_{a b}=\operatorname{diag}(1,-1,-1,-1)$. Round brackets and square brackets are used to represent the operations of symmetrization and anti-symmetrization on the indices, that is, $A_{(a b)}=\frac{1}{2}\left(A_{a b}+A_{b a}\right)$ and $A_{[a b]}=\frac{1}{2}\left(A_{a b}-A_{b a}\right)$.

The partial derivative with respect to the coordinate $x^{b}$ is denoted by a vertical bar, so that for a tensor $A^{a}$ it is

$$
\frac{\partial A^{a}}{\partial x^{b}}=A_{\mid b}^{a}
$$

and the covariant derivative with respect to $x^{b}$ is denoted by a double vertical bar, so that for the same tensor $A^{a}$ it becomes

$$
\frac{D A^{a}}{D x^{b}}=A_{\| b}^{a}=A_{\mid b}^{a}-\Gamma_{b c}^{a} A^{c}
$$

where the Christoffel symbols of the second kind $\Gamma_{b c}^{a}$ in terms of the metric $g_{a b}$ are given by

$$
\Gamma_{b c}^{a}=\frac{1}{2} g^{a m}\left(g_{m b \mid c}+g_{c m \mid b}-g_{b c \mid m}\right) .
$$

If $u^{a}=\frac{d x^{a}}{d \lambda}$ is the tangent to the curve $x^{a}(\lambda)$ parametrized by $\lambda$, the total derivative of $A^{a}$ along $x^{a}$ with respect to $\lambda$ is given by

$$
\frac{D A^{a}}{D \lambda}=A_{\| b}^{a} u^{b}=A_{\| b}^{a} \frac{d x^{b}}{d \lambda} .
$$

The Riemann curvature tensor $R_{a b c d}$ is defined via the relationship

$$
2 A_{b \|[c d]}=R_{a b c d} A^{a}
$$


and its contractions, the Ricci tensor and the scalar curvature, are $R_{a b}=R_{a c b}^{c}$ and $R=R_{a}^{a}$ respectively.

The Weyl or conformal curvature tensor $C_{a b c d}$ is defined through the decomposition of the Riemann curvature tensor given by

$$
R_{a b c d}=C_{a b c d}+\left(g_{a[c} R_{d] b}-g_{b[c} R_{d] a}-\frac{1}{3} R g_{a[c} g_{d] b}\right)
$$

\subsection{Light Propagation and Optical Scalars}

In order to study the propagation of light in general relativity, Maxwell's equations for the electromagnetic field must be written in a coordinate invariant form. This is achieved by representing the electromagnetic field as $F_{a b}$, an anti-symmetric covariant tensor field of rank 2. For a given point where $E_{\alpha}$ and $B_{\alpha}$ represent the Cartesian components of the electric and magnetic vector fields $\mathbf{E}$ and $\mathbf{B}$, these fields are related to $F_{a b}$ by

$$
F_{0 \alpha}=E_{\alpha} \text { and } F_{\alpha \beta}=-B_{\gamma}
$$

for $(\alpha, \beta, \gamma)$ a cyclic permutation of $(1,2,3)$. For an arbitrary metric $g_{a b}$, Maxwell's equations in vacuum are

$$
\begin{aligned}
F_{[a b \| c]} & =0, \\
F_{\| b}^{a b} & =0 .
\end{aligned}
$$

These equations cannot be solved explicitly except for highly symmetric situations and, unlike the classical case, plane wave solutions do not exist. However, it is possible to describe a short-wave (WKB) approximation, where the electromagnetic waves are nearly plane on scales that are large compared to the wavelength but small when compared to the radius of curvature of space-time. Under this approximation (see Section 3.2 in 
Schneider et al., 1992), possible solutions to (1.1) take the form

$$
F_{a b}=\operatorname{Re}\left[e^{i \frac{S}{\varepsilon}}\left(A_{a b}+\frac{\varepsilon}{i} B_{a b}\right)+\mathcal{O}\left(\varepsilon^{2}\right)\right]
$$

where $S$ represents the phase function and the term $A_{a b}+\frac{\varepsilon}{i} B_{a b}$ contains the information on the polarization and amplitude of the electromagnetic wave. The parameter $\varepsilon$ must be chosen to be small enough so that the phase $\frac{S}{\varepsilon}$ changes much faster than the amplitude $A_{a b}+\frac{\varepsilon}{i} B_{a b}$. By substituting this approximate solution (1.2) into Maxwell's equations (1.1) it is possible to calculate explicit transport laws for the polarization and amplitude that are consistent with the representation of the solutions as locally plane waves (see Section 3.2 in Schneider et al., 1992).

For an observer that follows a world line $x^{a}(\tau)$ with proper time $\tau$ and velocity $u^{a}=\frac{d x^{a}}{d \tau}$, the angular frequency of the wave is

$$
\omega=-\frac{d S}{d \tau}=-S_{\mid a} u^{a}
$$

where the wave vector can thus be defined as

$$
k_{a}=-S_{\mid a}
$$

Conditions on this wave vector can be found by substituting (1.2) into (1.1) and equating terms of equal order in $\varepsilon$. In particular, the wave vector is null so that

$$
k^{a} k_{a}=g^{a b} S_{\mid a} S_{\mid b}=0,
$$

which is the same as the eikonal equation from classical optics. The wavefronts are the hypersurfaces of constant phase, therefore $k^{a}$ is normal to the wavefronts and tangent to the local path of photons. Given these considerations, light rays are then described by 
the integral curves $x^{a}(\lambda)$ of $k^{a}$ given by

$$
\frac{d x^{a}}{d \lambda}=k^{a}
$$

where $\lambda$ is an affine parameter along the curve. Taking the covariant derivative of the tangent vector $k^{a}$ of a light ray along the ray $x^{a}(\lambda)$ it follows that

$$
k_{\| b}^{a} k^{b}=g^{a c} k_{c \| b} k^{b}=g^{a c} k_{b \| c} k^{b}=\frac{1}{2} g^{a c}\left(k^{b} k_{b}\right)_{\| c}=0
$$

where $k_{a \| b}=k_{b \| a}$ due to (1.4), and the eikonal equation (1.5) justifies the last step in (1.7). The equation $k_{\| b}^{a} k^{b}=0$ can be written more explicitly using (1.6) as

$$
\frac{d^{2} x^{a}}{d \lambda^{2}}+\Gamma_{b c}^{a} \frac{d x^{b}}{d \lambda} \frac{d x^{b}}{d \lambda}=0
$$

so that light rays are in fact null geodesics. Equation (1.8) implies that once the geometry of space-time is defined through a metric $g_{a b}$, thus defining the Christoffel symbols $\Gamma_{b c}^{a}$, the trajectories of photons or light rays can be established.

Besides being null solutions to the geodesic equation (1.8), another way to characterize light rays that is often useful in gravitational lensing theory is given in the theorem known as Fermat's principle:

Theorem (Fermat's Principle). Consider a source event $S$ and an observer described by a time-like world line $\ell$ in a space-time defined by a metric $g_{a b}$ over a manifold $M$. A smooth null curve $\gamma$ from $S$ to $\ell$ is a light ray or null geodesic if, and only if, its arrival time $\tau$ on $\ell$ is stationary under first-order variations, that is,

$$
\delta \tau=0
$$

Proofs of this theorem can be found in Section 3.3 of Schneider et al. (1992) or in 
Perlick (1990).

The description of light rays using the geodesic formalism or Fermat's principle requires tracing the path of individual photons as they travel through space-time. However, for astrophysically relevant observables such as flux, luminosity, angular size and redshift derived from flux, this entails ray-tracing of a set of photon paths instead of a single ray. The optical scalar formalism provides a way to follow the evolution and propagation of a bundle of rays without having to trace the path of each individual ray.

Consider a ray $\gamma$ as the central ray of an infinitesimally thick bundle of rays. A secondary ray $\gamma^{\prime}$ in the bundle is separated from the central ray $\gamma$ by a vector $\delta x^{a}$. If the rays $\gamma$ and $\gamma^{\prime}$ are required to have the same phase, then

$$
k_{a} \delta x^{a}=0,
$$

for $k_{a}$ the wave vector of $\gamma$ given as in (1.4). A collection of infinitesimally close rays such that (1.10) holds is called a beam. The interest is then the study of the evolution and transport of the cross-section of a beam with respect to the central ray or a parameter for the central ray. It is then convenient to introduce a null tetrad to analyze the evolution of vectors in the beam. ${ }^{1}$ Starting with $k^{a}$ as a null vector, there is another linearly independent null vector $l^{a}$ and it is possible to introduce two more complex null vectors $m^{a}$ and $\bar{m}^{a}$ which are complex conjugates of each other. The system forms a null tetrad if all possible scalar products are zero with the exception of

$$
k^{a} l_{a}=1 \quad \text { and } \quad m^{a} \bar{m}_{a}=-1 .
$$

It is possible to visualize $m^{a}$ and $\bar{m}^{a}$ as spanning the plane $\pi$, where the cross-section of the beam lies, orthogonal to the ray direction. Thus, the main question for the evolution

\footnotetext{
${ }^{1}$ Tetrads and null tetrads are discussed in most textbooks on General Relativity. See for example Sections 17.3 and 18.2 in Stephani (1990) or Section 13.2, page 373 in Wald (1984).
} 
of the beam turns into a study of how does the projection of $\delta x^{a}$ onto $\pi$ change as a function of an affine parameter $\lambda$ for the ray $\gamma$.

The projection tensor

$$
p_{a b}=\bar{t}_{a} t_{b}+t_{a} \bar{t}_{b}
$$

can be used to obtain components of vectors on the plane $\pi$. Given that in terms of the null tetrad the metric has the form

$$
g_{a b}=m_{a} \bar{m}_{b}+\bar{m}_{a} m_{b}+k_{a} l_{b}+l_{a} k_{b}
$$

then the projection tensor is

$$
p_{a b}=g_{a b}-k_{a} l_{b}-l_{a} k_{b}
$$

The projection of $\delta x^{a}$ onto $\pi$ is then $\delta_{\perp} x^{a}=p^{a}{ }_{b} \delta x^{b}$ and the change of $\delta_{\perp} x^{a}$ along the ray $\gamma$ when projected back onto $\pi$ is given by

$$
\Delta^{a}=p_{b}^{a}\left(p_{c}^{b} \delta x^{c}\right)_{\| d} k^{d}
$$

It is also possible to show that the covariant transport of $\delta x^{a}$ along the ray is given by

$$
\left(\delta x^{a}\right)_{\| b} k^{b}=k_{\| b}^{a} \delta x^{b}
$$

so that using (1.10), (1.16) and the orthogonality properties of the null tetrad, equation (1.15) for $\Delta^{a}$ becomes

$$
\Delta^{a}=\theta \delta_{\perp} x^{a}+i \omega\left(m^{a} \bar{m}_{b}-\bar{m}^{a} m_{b}\right) \delta_{\perp} x^{b}-2 \operatorname{Re}\left(\sigma \bar{m}^{a} \bar{m}_{b}\right) \delta_{\perp} x^{b}
$$


where the optical scalars $\theta, \omega$ and $\sigma$ are given $b^{2}$

$$
\begin{aligned}
\theta & =\frac{1}{2} k_{\| a}^{a} \\
\omega & =\sqrt{\frac{1}{2} k_{[a \| b]} k^{a \| b}} \\
|\sigma| & =\sqrt{\frac{1}{2}\left(k_{(a \| b)} k^{a \| b}-\frac{1}{2}\left(k_{\| c}^{c}\right)^{2}\right)} .
\end{aligned}
$$

Since according to the definition (1.4), $k^{a}$ is a gradient, it follows that $k_{a \| b}=k_{b \| a}$ and therefore $\omega$ vanishes as it is related to the antisymmetric part of $k_{a \| b}$ and a measure of the rotation of the beam. In equation (1.17) the term that depends on $\theta$ is a measure of the isotropic expansion of the beam and the term that depends on $\sigma$, being related to the traceless symmetric part of $k_{a \| b}$, represents the shear of the beam as a circular cross-section is deformed into an ellipse with $|\sigma|$ as the ratio of the axes.

Using Ricci's identity for the curvature tensor,

$$
k_{a \| b c}-k_{a \| c b}=R_{d a b c} k^{d}
$$

the transport equations for the optical scalars (1.18) are obtained

$$
\begin{aligned}
\dot{\theta}+\theta^{2}+|\sigma|^{2} & =\frac{1}{2} R_{a b} k^{a} k^{b} \\
\dot{\sigma}+2 \theta \sigma & =\frac{1}{2} C_{a b c d} k^{a} \bar{m}^{b} k^{c} m^{d}
\end{aligned}
$$

where the dot ${ }^{\cdot} \equiv \frac{d}{d \lambda}$ denotes the derivative with respect to the affine parameter for the ray as defined in (1.6). If $A$ is the area of the beam, given the definition of $\theta$, it follows that $\sqrt{A}=2 \theta \sqrt{A}$, and the transport equations for the optical scalars in (1.19) can be rewritten as

$$
\begin{aligned}
\frac{\ddot{\sqrt{A}}}{\sqrt{A}}+|\sigma|^{2} & =\frac{1}{2} R_{a b} k^{a} k^{b} \\
\frac{(\sigma A)^{\cdot}}{A} & =\frac{1}{2} C_{a b c d} k^{a} \bar{m}^{b} k^{c} m^{d},
\end{aligned}
$$

\footnotetext{
${ }^{2}$ For more details on the optical scalar treatment, see Section 17.3, page 180 in Stephani (1990) or Section 3.4.2 in Schneider et al. (1992).
} 
where the first of these relationships is also known as the focusing equation.

\subsection{Cosmology and Distances}

Summarized in this section is the basic background and relationships in cosmology that will be used later in the context of gravitational lensing. For more details, the textbooks on cosmology by Peebles (1993) or Peacock (1999) are recommended.

General Relativity asserts that the presence of matter affects the geometry of spacetime. However, changes in the geometry of space-time will affect the matter configuration which in turn will change the geometry and so on, illustrating the non-linear nature of General Relativity. Furthermore, the theory is overdetermined, a fact that when coupled with the non-linearity makes the finding of solutions to Einstein's field equations a rather complicated task. In particular, there is no known solution that describes the observed Universe at all scales.

A first approximation to a description of the Universe stems from observations of the distribution of galaxies and high redshift sources. Based on the averaged distribution over large angles, the Universe is isotropic. Furthermore, if the Cosmological Principle is accepted in that our location in the Universe is not privileged, then isotropy is true for all observers and thus the Universe is homogeneous as well. ${ }^{3}$ In general relativistic terms, isotropy implies a metric with spherical symmetry while homogeneity implies that spatial sections have constant curvature that can depend on time. A metric that satisfies these conditions is called a Robertson-Walker metric (after Robertson, 1935 and Walker, 1935), and has a space-time interval that can be written in the form

$$
d s^{2}=c^{2} d t^{2}-R^{2}(t)\left[d \omega^{2}+f_{k}^{2}(\omega) d \Omega^{2}\right]
$$

\footnotetext{
${ }^{3}$ This is a great simplification, true only when observations are averaged over large scales. Incidentally, gravitational lensing would not be present in a homogeneous universe.
} 
where $t$ is the cosmic time or the time measured by comoving observers, that is, observers that move with the cosmological flow so that positions of cosmological objects are given by fixed coordinates and the expansion is entirely due to the scale factor $R(t)$. In equation (1.21), $d \Omega$ is the solid angle interval for the angular coordinates $(\theta, \varphi)$ on a unit sphere, $\omega$ is the comoving dimensionless radial coordinate and $f_{k}(\omega)$ is a function that depends on the curvature $k$ of the spatial sections of constant curvature, given by

$$
f_{k}(\omega)=\left\{\begin{array}{lll}
\sin (\omega) & k=1 & \text { positive curvature } \\
\omega & k=0 & \text { flat } \\
\sinh (\omega) & k=-1 & \text { negative curvature }
\end{array}\right.
$$

For a given space-time interval $d s^{2}$, the metric tensor $g_{a b}$ is determined through

$$
d s^{2}=g_{a b} d x^{a} d x^{b}
$$

where $\left(x^{0}, x^{1}, x^{2}, x^{3}\right)=(c t, \omega, \theta, \varphi)$.

Substituting the metric (1.21) into Einstein's field equations given by

$$
R^{a b}-\frac{1}{2} R g^{a b}+\Lambda g^{a b}=-\frac{8 \pi G}{c^{4}} T^{a b}
$$

where $\Lambda$ is the cosmological constant, then the matter content described by the energy momentum tensor $T^{a b}$ must be that of a homogeneous perfect fluid with pressure $p(t)$ and density $\rho(t)$. For the case of matter particles with random thermal velocities that are much smaller than $c$, the contribution of the pressure terms is negligible and the matter content is said to be described by a pressureless dust. Under the assumption of negligible pressure, Einstein's field equations (1.22) for the metric (1.21) turn into Friedmann's 
equation (see Friedmann, 1999)

$$
\left(\frac{\dot{R}}{R}\right)^{2}=\frac{8 \pi G \rho}{3}-\frac{k c^{2}}{R^{2}}+\frac{\Lambda c^{2}}{3}
$$

and a simplified equation for adiabatic expansion (see Lemaître, 1931)

$$
\left(\rho R^{3}\right)^{\cdot}=0
$$

where, unlike in equation (1.19), the dot ${ }^{*} \equiv \frac{d}{d t}$ denotes the time derivative instead of the derivative with respect to an affine parameter. Note that given values for density $\rho$, curvature $k$ and cosmological constant $\Lambda$, equations (1.23) and (1.24) determine the evolution of the scale factor $R(t)$. Cosmological models with a Robertson-Walker metric (1.21) that obey the Friedmann-Lemaitre equations (1.23) and (1.24) are called FLRW models.

Dimensionless parameters can be used to represent the evolution of the scale factor $R(t)$. The density parameter $\Omega_{M}$ and the cosmological constant parameter $\Omega_{\Lambda}$ are defined by

$$
\begin{aligned}
\Omega_{M} & \equiv \frac{8 \pi G \rho}{3 H^{2}}=\frac{\rho}{\rho_{\text {crit }}} \\
\Omega_{\Lambda} & \equiv \frac{\Lambda c^{2}}{3 H^{2}}
\end{aligned}
$$

where $\rho_{\text {crit }} \equiv \frac{3 H^{2}}{8 \pi G}$ is the critical density for a universe with $\Lambda=0$ and $k=0$, and the Hubble parameter $H$ is defined as

$$
H \equiv\left(\frac{\dot{R}}{R}\right)
$$

The dimensionless deceleration parameter $q$ is given by

$$
q \equiv-\left(\frac{\ddot{R} R}{(\dot{R})^{2}}\right)
$$


and a dimensionless curvature parameter $\Omega_{k}$ can be defined as

$$
\Omega_{k} \equiv-\frac{k c^{2}}{H^{2} R^{2}}
$$

In terms of the parameters defined in (1.25), (1.26), (1.27) and (1.28) the evolution of the scale factor represented in the Friedmann-Lemaître equations (1.23) and (1.24) can be written in a simpler fashion as

$$
\begin{aligned}
1 & =\Omega_{M}+\Omega_{\Lambda}+\Omega_{k} \\
q & =\frac{\Omega_{M}}{2}-\Omega_{\Lambda} .
\end{aligned}
$$

From the frequency of a light ray (1.3) that travels in a space-time described by the Robertson-Walker metric (1.21), radiation that a source $S$ emits at time $t_{S}$ and is seen by an observer $O$ at time $t_{O}$ has a relative change in wavelength given by the redshift

$$
z \equiv \frac{\lambda_{O}-\lambda_{S}}{\lambda_{S}}=\frac{R\left(t_{O}\right)}{R\left(t_{S}\right)}-1
$$

where $\lambda_{S}$ is the emission wavelength at the source and $\lambda_{O}$ is the wavelength seen by the observer.

In General Relativity's curved space-time the term "distance" is not a properly defined quantity as different experiments that on Euclidean space would agree on the same result now give different results for a quantity that can only be determined indirectly. Since our aim is to work with light rays, the working definition for distance in gravitational lensing should be operationally set in terms of the propagation of light beams. The quantity most relevant for lensing theory is thus the angular diameter distance. For an object of diameter or physical size $\delta d$ that, when observed subtends and angle $\delta \theta$, the angular diameter distance $D$ is given by

$$
D=\frac{\delta d}{\delta \theta}
$$


If the comoving radial coordinate of the object is $\omega$, then according to (1.21) the size of the object is $\delta d=R(t) f_{k}(\omega) \delta \theta$ and thus the angular diameter distance becomes

$$
D=R(t) f_{k}(\omega)
$$

This last equation must be integrated using (1.23) or (1.29) in order to obtain the angular diameter distance $D$ in terms of the observable redshift $z$. The angular diameter distance $D(z)$ derived from equation (1.32), although useful in instances where the Universe can be described by an FLRW model, is not suitable for gravitational lenses since homogeneity and isotropy are clearly broken by the presence of the lens.

The optical scalar formalism for the propagation of light beams discussed in Section 1.2 can be used to derive relationships for the angular diameter distance that include the effects of more generic metrics. Consider a light beam of cross-section area $A$ that subtends a solid angle $\delta \Omega$ at the position of an observer. Then the angular diameter distance (1.31) of a source of physical size $\delta d$ that subtends an angle $\delta \theta$ and spans the cross-section area of the beam is given by

$$
D=\sqrt{\frac{A}{\delta \Omega}} .
$$

Using the transport equations for the optical scalars in terms of the cross-section area of the beam (1.20) and the angular diameter distance (1.33), the equations for the angular diameter distance as a function of the affine parameter $\lambda$ of the light rays take the form of a set of differential equations

$$
\begin{aligned}
\frac{d^{2} D}{d \lambda^{2}}+|\sigma| D & =D \mathcal{R} \\
\frac{d\left(\sigma D^{2}\right)}{d \lambda} & =D^{2} \mathcal{F} e^{i \beta}
\end{aligned}
$$


where $\mathcal{R}$ and $\mathcal{F}$ are the Ricci and Weyl driving terms defined by

$$
\begin{aligned}
\mathcal{R} & =\frac{1}{2} R_{a b} k^{a} k^{b} \\
\mathcal{F} e^{i \beta} & =\frac{1}{2} C_{a b c d} k^{a} \bar{m}^{b} k^{c} m^{d} .
\end{aligned}
$$

The limiting case where the shear approaches zero is of particular interest in gravitational lensing as it describes the propagation of the beam when it is far from lensing masses. Using Einstein's field equations (1.22) it follows that $R^{a b}=-\frac{8 \pi G}{c^{4}} T^{a b}+\frac{1}{2} R g^{a b}-$ $\Lambda g^{a b}$, so that the Ricci driving term $\mathcal{R}$ in (1.35) becomes

$$
\mathcal{R}=-\frac{4 \pi G}{c^{4}} T_{a b} k^{a} k^{b}
$$

Now, the case of a non-homogeneous universe can be described by a smoothness parameter $\alpha$ that represents the fraction of matter that is smoothly distributed, while $1-\alpha$ is the fraction of matter bound in galaxies (see Dyer, 1973 and Dyer and Roeder, 1973). A beam that propagates far from bound matter exhibits no shear and the Ricci driving term becomes

$$
\mathcal{R}=-\frac{4 \pi G}{c^{4}} T_{00} k^{0} k^{0}=-\frac{4 \pi G}{c^{4}} \alpha \rho k^{0} k^{0}
$$

Furthermore, for the Robertson-Walker metric (1.21) it is possible to express $k^{0}$ as

$$
k^{0} \equiv c \frac{d t}{d \lambda}=\frac{c}{H_{0}} \frac{R_{0}}{R}=\frac{c}{H_{0}}(1+z)
$$

where $R_{0}$ and $H_{0}$ are the present values of the scale and Hubble parameters respectively. Given the equation for adiabatic expansion (1.24) it follows that $\rho R^{3}=\rho_{0} R_{0}^{3}$ and therefore equation (1.37) can be rewritten as

$$
\mathcal{R}=-\frac{4 \pi G}{c^{4}} \alpha \rho_{0}\left(\frac{c}{H_{0}}\right)^{2}(1+z)^{5} .
$$


Thus, the focusing equations for the evolution of the angular diameter distance (1.34) in the zero shear case simplify to

$$
\frac{d^{2} D}{d \lambda^{2}}+\frac{3}{2} \alpha \Omega_{M, 0}(1+z)^{5} D=0,
$$

where $\Omega_{M, 0}$ is the present value of the density parameter $\Omega_{M}$. Now, in order to obtain a relationship for the distance $D$ as a function of redshift $z$ as was done in the case of a FLRW model, the affine parameter $\lambda$ must be written in terms of the redshift. From equations (1.30), (1.23) and (1.24) it follows that

$$
\frac{d \lambda}{d z}=(1+z)^{-3}\left(\Omega_{M, 0}(1+z)+\Omega_{\Lambda, 0}(1+z)^{-2}+1-\Omega_{M, 0}-\Omega_{\Lambda, 0}\right)^{-\frac{1}{2}}
$$

where $\Omega_{\Lambda, 0}$ is the present value of the cosmological constant parameter $\Omega_{\Lambda}$. Combining (1.39) and (1.40) a differential equation for the angular diameter distance as a function of redshift is obtained

$$
\begin{aligned}
{\left[(1+z)\left(\Omega_{\Lambda, 0}+\left(1-\Omega_{M, 0}-\Omega_{\Lambda, 0}\right)(1+z)^{2}+\Omega_{M, 0}(1+z)^{3}\right)\right] \frac{d^{2} D}{d z^{2}} } & + \\
{\left[2 \Omega_{\Lambda, 0}+3\left(1-\Omega_{M, 0}-\Omega_{\Lambda, 0}\right)(1+z)^{2}+\frac{7}{2} \Omega_{M, 0}(1+z)^{3}\right] \frac{d D}{d z} } & + \\
{\left[\frac{3}{2} \alpha \Omega_{M, 0}(1+z)^{2}\right] D } & =0 .
\end{aligned}
$$

This equation must be integrated to obtain $D(z)$ and is known as the Dyer-Roeder equation (see Dyer and Roeder, 1972, 1973, 1974, 1981b).

\subsection{Light Bending and the Lens Equation}

According to General Relativity, light rays propagate along the null geodesics of a metric solution of Einstein's Field Equations. However, when matter is localized in a region that is small compared to the distance between source and observer, a simplified approach is possible. Under this ray tracing approximation, the effect of a mass distribution on a 
light ray is a localized bending or deflection of the ray from its original direction.

A light ray that passes by the exterior of a spherical mass $M$ at a minimum distance $\xi$ from the center of the object has its trajectory deflected by an angle

$$
\alpha=\frac{4 G M}{c^{2} \xi},
$$

known as the Einstein angle. Equation (1.42) is valid as long as the weak field approximation holds in the vicinity of the minimum distance $\xi$. Thus, equation (1.42) can be used as long as the impact parameter $\xi$ is much larger than the Schwarzschild radius $R_{S}$ of the mass $M$ given by

$$
R_{S}=\frac{2 G M}{c^{2}}
$$

Note that the condition $\xi \gg R_{S}$ implies that the deflection angle is small and the equivalent Newtonian potential obeys $\phi \ll c^{2}$. The result for the bending angle (1.42) can be derived using the linearized Schwarzschild metric as found in most textbooks on General Relativity (see for example Section 8.5 in Weinberg, 1972, Exercise 18.6 in Misner et al., 1973 or Section 10.5 in Stephani, 1990). From here onwards, the term point mass will be used when dealing with rays at the exterior of a spherical mass distribution with a radius large enough that possible impact parameters are much larger than the Schwarzschild radius of the mass.

The deflection angle (1.42) leads to a simple gravitational lensing configuration illustrated in Figure 1.1, known as the Schwarzschild lens. The point mass $M$ acts as a gravitational lens for rays from source $S$ to observer $O$. The line $\overline{O M}$ defines an arbitrary optical axis for the measurement of angles and distances. Note that due to the spherical symmetry of the mass distribution, light rays from the source to the observer must lie on the plane defined by the source, lens and observer. This implies that $\eta$, the position of the source with respect to the optical axis on a plane that contains the source and 


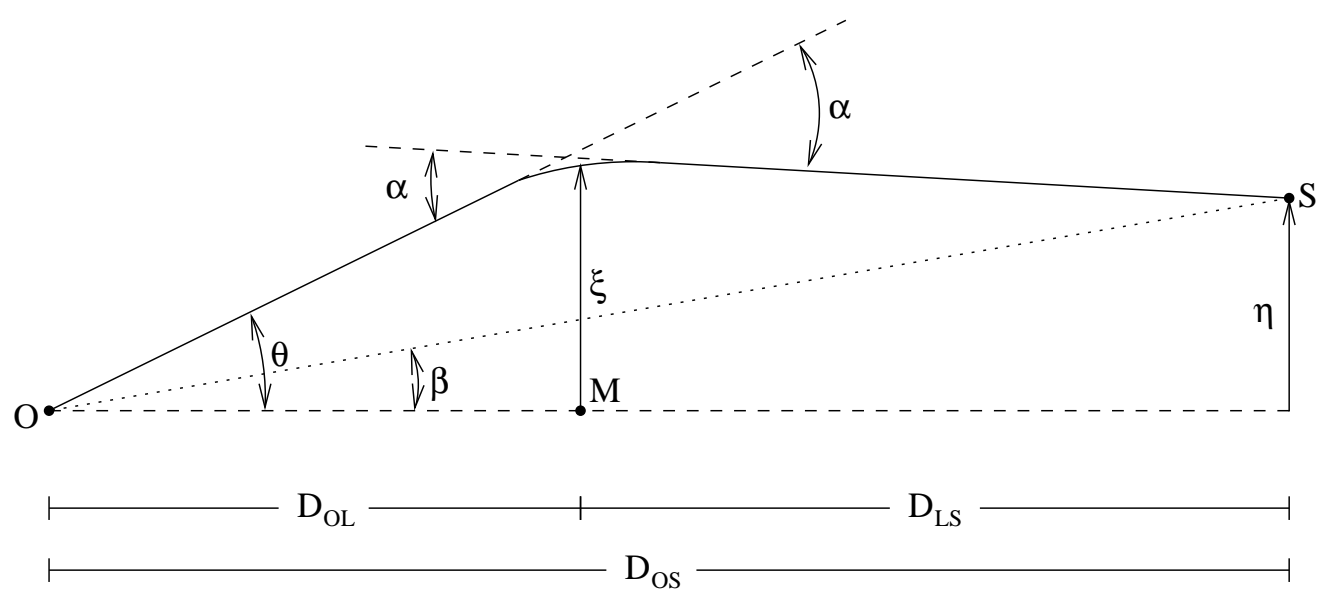

Figure 1.1: A point mass $M$ at a distance $D_{O L}$ from observer $O$, deflects a ray from source $S$ that passes at a distance $\xi$ from the point mass. Due to the deflection of the ray from source to observer by an angle $\alpha$, the source has its real angular position $\beta$ observed to be $\theta=\frac{\xi}{D_{O L}}$. All angles are small and have been exaggerated in the figure.

is perpendicular to the optical axis, ${ }^{4}$ instead of being a two dimensional vector can be simplified to one dimension. Similarly, the position where the light ray impacts the plane that contains the point mass and is perpendicular to the optical axis, ${ }^{5}$ given by $\xi$, can also be treated as a one-dimensional quantity. The distances in Figure 1.1 from observer to source, from observer to lens and from lens to source are noted as $D_{O S}, D_{O L}$ and $D_{L S}$ respectively, and following the discussion regarding distances from Section 1.2, are assumed to be angular diameter distances.

Let $\beta$ denote the angular position of the unlensed source with respect to the optical axis, $\theta$ the angular position of the deflected ray with respect to the optical axis and $\alpha$ the deflection for a light ray that passes a minimum distance $\xi$ from the lens $M$ given by equation (1.42). Under the assumption that the region where the deflection occurs is small or, equivalently speaking, the size of the lens is very small when compared to the distance from observer to source, the lens is said to be geometrically thin. Under the condition that the deflected ray reaches the observer and since the angles are small, the

\footnotetext{
${ }^{4}$ This plane is called the source plane

${ }^{5}$ This plane is called the lens plane
} 
geometry of Figure 1.1 implies that

$$
\beta=\theta-\frac{D_{L S}}{D_{O S}} \alpha
$$

or in terms of the distances on source and lens planes with respect to the optical axis

$$
\eta=\frac{D_{O S}}{D_{O L}} \xi-D_{L S} \alpha
$$

Equations (1.44) and (1.45) are called the lens equation and represent a transformation between the source plane and the lens plane where the position of the source $\eta$ or $\beta$ is distorted by the presence of the mass $M$ and instead observed on the lens plane as an image located at $\xi$ or $\theta$. Using the deflection angle (1.42) in the lens equation (1.45), a relationship between the position of the source in the source plane and the impact parameter on the lens plane can be written with explicit dependence on the deflecting mass $M$

$$
\eta=\frac{D_{O S}}{D_{O L}} \xi-\frac{4 G M}{c^{2}} \frac{D_{L S}}{\xi}
$$

This equation is to be solved to find the true position of a source $\eta$ when the position of an image $\xi$ is known or conversely, to find the position $\xi$ of images, ${ }^{6}$ given the location of a source at $\eta$. The simple case of a point mass already indicates that the lens equation (1.46) is not linear and, save for some very simple cases of mass models, analytic inversion will not be possible.

For the case of a transparent spherical mass distribution, where light rays can pass through the mass distribution and thus images can form and be seen inside the profile of the extended source, the deflection angle is

$$
\alpha=\frac{4 G \tilde{m}(\xi)}{c^{2} \xi}
$$

\footnotetext{
${ }^{6}$ This simple case of point-mass illustrates that the lens map between source and lens plane describes the production of multiple images of a single source.
} 
where $\tilde{m}(\xi)$, called the cylindrical mass, is the mass inside a cylinder of radius $\xi$ with center at the center of the mass distribution and axis parallel to the line between observer and the center of the distribution. The deflection angle (1.47) can be derived either using the optical scalar formalism detailed in Section 1.2 (see Clark, 1972 or Dyer, 1977) or using the linearized field equations (see Clark, 1972).

The basic geometry of this transparent spherical mass distribution acting as a gravitational lens is the same as the one for a point-mass described in equations (1.44) and (1.45) and illustrated in Figure 1.1. The only difference is that the deflection angle now has the form (1.47) so that the lens equation can be written as

$$
\eta=\frac{D_{O S}}{D_{O L}} \xi-\frac{4 G D_{L S}}{c^{2}} \frac{\tilde{m}(\xi)}{\xi}
$$

Note that if the mass distribution has finite radius, solutions of the lens equation (1.48) that lie outside this radius will be the same as those of a point lens of mass equal to the total mass of the extended lens. This implies that only images inside the extended mass will be different from those of the point-mass case, a fact that will be exploited later when extending results from point lenses to distributed ones.

A more generic mass distribution either in the form of a single non-spherical profile or as a collection of several deflecting masses causes a bending that can be similarly calculated under the conditions that the weak field approximation holds in the regions where light rays will be passing near the mass distributions, ${ }^{7}$ that the distribution is quasi-stationary so that speeds of matter in the lens are much smaller than $c$ and that the mass distribution is geometrically thin so that the extent of the mass in the direction of propagation of the light rays is small when compared to the distance between observer and lens. The last requirement for the lens to be thin is equivalent to the requirement that the component of the gravitational field transverse to the direction of propagation of the

\footnotetext{
${ }^{7}$ And in consequence the bending angle will be small.
} 
actual ray can be approximated by the same component of the field on the unperturbed ray.

Using either Fermat's principle (1.9) or the linearized field equations formalism along with the null geodesic equation (1.8), it is possible to show that for a geometrically thin mass distribution that can be described by a projected mass density $\sigma(\boldsymbol{\xi})$ on the lens plane, the resulting bending is the vector addition of the Einstein angles integrated over the lens plane. This means that a generic, yet thin mass distribution causes a bending given by

$$
\boldsymbol{\alpha}(\boldsymbol{\xi})=\frac{4 G}{c^{2}} \int_{\mathbb{R}^{2}} \frac{\left(\boldsymbol{\xi}-\boldsymbol{\xi}^{\prime}\right) \sigma\left(\boldsymbol{\xi}^{\prime}\right)}{\left|\boldsymbol{\xi}-\boldsymbol{\xi}^{\prime}\right|^{2}} d^{2} \xi^{\prime}
$$

where, given that the mass distribution is no longer spherically symmetric, the impact parameter $\boldsymbol{\xi}$ and the deflection angle $\boldsymbol{\alpha}$ are two-dimensional vector quantities. The impact parameter $\boldsymbol{\xi}$ is measured with respect to the center of the surface mass density $\sigma(\boldsymbol{\xi})$ and this center then defines the optical axis of the configuration as the line between the observer and the center. ${ }^{8}$

The geometry of the basic lens situation is similar to that of Figure 1.1. However, the breaking of the symmetry implies that rays from observer to source do not necessarily lie on the plane formed by lens, observer and source. As a consequence, angles $\boldsymbol{\beta}, \boldsymbol{\theta}$ and $\boldsymbol{\alpha}$ must be described by two-dimensional vectors and the position of the source $\boldsymbol{\eta}$ and the position of an image or impact parameter $\boldsymbol{\xi}$ are described by two-dimensional vectors on the source plane and lens plane respectively. The new configuration is illustrated in Figure 1.2.

Using the bending angle (1.49) and the geometry from Figure 1.2, provided that the angles are small and the distances are angular diameter distances, the transformation that relates source position on the source plane with the position of observed images or

\footnotetext{
${ }^{8}$ The choice of center for the mass distribution is essentially arbitrary and can be the centroid or the center of mass or any other point inside the mass distribution.
} 


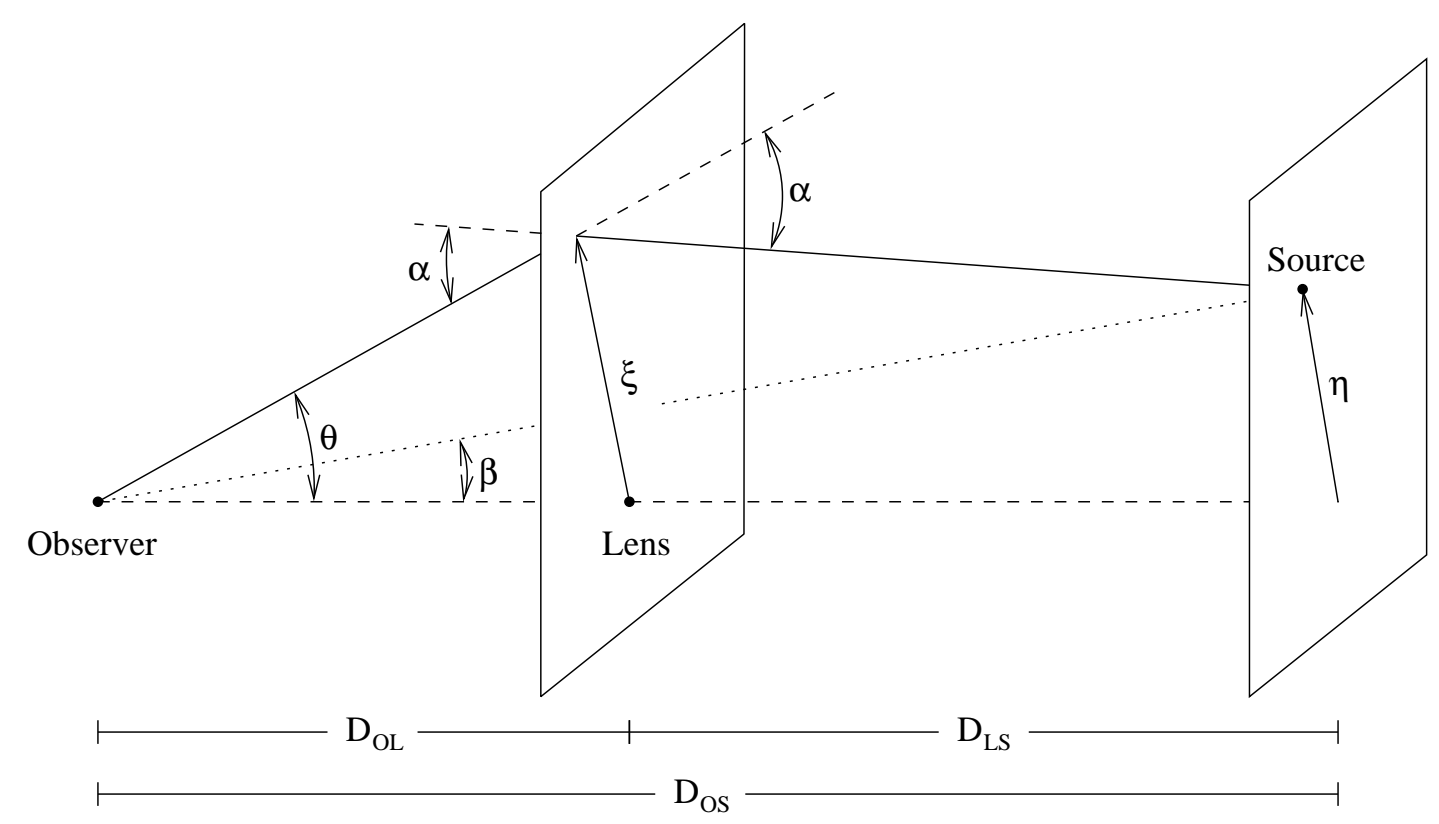

Figure 1.2: A mass distribution at a distance $D_{O L}$ from the observer deflects by an angle $\boldsymbol{\alpha}$ a ray that hits the lens plane at $\boldsymbol{\xi}$. The source, at a distance $D_{O S}$ from the observer and located on the source plane at $\boldsymbol{\eta}$, has its real angular position $\boldsymbol{\beta}$ observed to be $\boldsymbol{\theta}=\frac{\boldsymbol{\xi}}{D_{O L}}$ due to the gravitational lens. All angles are small and have been exaggerated in the figure.

impact parameters for light rays on the lens plane, is given by the lens equation

$$
\boldsymbol{\eta}=\frac{D_{O S}}{D_{O L}} \boldsymbol{\xi}-D_{L S} \boldsymbol{\alpha}(\boldsymbol{\xi})
$$

The last form of the lens equation (1.50) can be derived using Fermat's principle (1.9) applied to the time delay for a ray affected by the lensing system when compared to a ray in the absence of lensing (see Sections 4.3 and 4.6 in Schneider, 1985). This time delay can be calculated in two different ways. Following Cooke and Kantowski (1975), the time delay can be separated into two parts; a part containing the information of the difference in path lengths that depends on the geometry of the background space-time called the geometrical time delay, and a part containing the information of the retarding effect of the gravitational field near the lens on the ray that is called the potential time delay. ${ }^{9}$ It is also possible to obtain the time delay by studying the distortion effect of the

\footnotetext{
${ }^{9}$ The potential time delay arises from the retardation on the rays due to the gravitational field and is known as the Shapiro effect (see Shapiro, 1964).
} 
gravitational lensing potential on the wavefronts that describe the propagation of light rays from the source (see Kayser and Refsdal, 1983).

If the distances in equation (1.50) are angular diameter distances (see Section 1.3), that include the effects of inhomogeneity in the matter distributions as described by the smoothness parameter in the Dyer-Roeder equation (1.41), as well as the effects of redshift due to the evolution of the matter dominated Friedmann-Lemaitre RobertsonWalker background metric, then the lens equation (1.50) is valid in a cosmological context where redshifts are not constrained to be small.

\subsection{Properties of the Lens Equation}

The lens equation (1.50) represents a transformation between the plane where a luminous source is located and the plane where the deflectors or lenses are located. These planes are defined with respect to an arbitrary optical axis formed by the observer and a point on the lensing mass distribution. For a given position of the source $\boldsymbol{\eta}$, solutions of the lens equation (1.50) for $\boldsymbol{\xi}$ represent images observed.

A study on the differential and topological properties of the lens equation is thus required in order to understand the details of image production, multiple imaging and maximal lensing.

Let $\mathbf{J}$ represent the Jacobian matrix of the lens equation as a transformation between the source and lens planes. That is,

$$
\mathbf{J}=\frac{\partial \boldsymbol{\eta}}{\partial \boldsymbol{\xi}}
$$

As the Jacobian matrix describes the distortion of areas from source plane to lens plane, it then contains the information on the change of the shape of an image with respect to the shape of the source. Furthermore, unlike the lens transformation (1.50), the Jacobian matrix (1.51) is analytic, since the deflection angle (1.50) is itself an analytic function 
provided that the mass density decreases faster than $r^{-2}$ as $r \rightarrow \infty$ and diverges slower than $r^{-1}$ as $r \rightarrow 0$, for $r$ measured with respect to the center of the mass distribution. The analyticity of the Jacobian matrix (1.51) simplifies the study of the differential properties of the lens equation (1.50) and thus helps in the study of image production.

If an infinitesimal source subtends a solid angle $\delta \Omega_{S}$ and, due to a gravitational lens, the corresponding image subtends a solid angle $\delta \Omega_{I}$, given that the surface brightness must be conserved, ${ }^{10}$ the ratio of the flux ${ }^{11}$ of the image to the flux of the unlensed source, or magnification, is given by

$$
\mu=\frac{\delta \Omega_{I}}{\delta \Omega_{S}}
$$

Since the solid angle subtended by the light beam of a source or image is proportional to the area of the beam, a relationship between the magnification (1.52) and the Jacobian of the lens transformation (1.51) can be established. If a source at $\boldsymbol{\eta}$ that subtends a solid angle $\delta \Omega_{S}$ is lensed to an image at $\boldsymbol{\xi}$ that subtends a solid angle $\delta \Omega_{I}$, then

$$
\mu=\frac{\delta \Omega_{I}}{\delta \Omega_{S}}=|J|^{-1}
$$

where $J=\operatorname{det} \mathbf{J}$. This relationship between the Jacobian and the magnification $\mu$ is the reason why $\mathbf{J}$ is called the magnification matrix. ${ }^{12}$

Points on the lens plane where $J=0$ form closed disjoint smooth curves called critical curves (see Section 6.2 in Schneider et al., 1992 and Section 6.2.4, page 183 in Petters et al., 2001). Critical curves then separate regions in the lens plane where $J$ has opposite parity and images are assigned a parity according to the sign of $J$. It is worth noting

\footnotetext{
${ }^{10}$ Photon number conservation (see Section 3.2, page 99 and Section 5.2 in Schneider et al., 1992) or an argument using Liouville's theorem (see Section 2.3 in Schneider et al., 2006) can be employed to show that gravitational lensing conserves surface brightness.

${ }^{11}$ The flux is the product of the surface brightness and the subtended solid angle and is one of the astrophysical observables in gravitational lens systems.

${ }^{12}$ The magnification or increase in observed flux of an image over that of the unlensed source caused by gravitational lensing is the reason why lensing allows for the observation of very faint and distant objects (see for example Hu et al., 2002, Kneib et al., 2004 or Pelló et al., 2004).
} 
that even though formally at a point where $J=0$ the image has infinite magnification, this is not observed for two reasons. First, real sources are not points but are extended in size and thus the magnification is the weighted mean over the source, leading to finite magnifications. Second, the derivations of the light bending and the lens equation have been made under the assumptions of geometrical optics, but this approximation breaks down in the vicinity of critical points and thus wave optics must be employed. Once wave optics are introduced in the calculations, point sources can be shown to produce finite magnifications (see Section 7.2 in Schneider et al., 1992).

The image of all points in the critical curves under the lens transformation forms a set of curves on the source plane called caustics. ${ }^{13}$

Since the lens transformation (1.50) is invertible in a neighborhood of any point with $J \neq 0$, a change in the position of the source will only lead to a change in the number of images if the source crosses a caustic. Given that the number of images produced by a gravitational lens must be odd (see Chapter 2, Section 2.2, and references therein), then when the source crosses a caustic, a pair of images is created or destroyed.

The close connection between the number of images of a gravitational lens and the respective caustics, critical curves and magnification matrix of the lens map highlights the relevance of the study of these differential properties when looking for results in maximal lensing. Studies in the number of cusps in caustics (see Witt and Petters, 1993 and Petters, 1995b) and the classification of caustics for systems with multiple images (see Keeton et al., 2000) have given insight and led the way to results in image counting that will be studied in the next chapter.

\footnotetext{
${ }^{13}$ Caustics, unlike critical curves, are not always smooth and cusps are a very common feature in them (see Section 2.4 in Schneider et al., 2006).
} 


\section{Chapter 2}

\section{Image Counting}

Having outlined the foundations of gravitational lensing theory in the previous chapter, in this chapter we present the most important work and results in the area of multiple imaging and image counting.

The problem of inverting the lens equation in order to find the positions of all images in terms of the source position is not a trivial one. That is, given a source and lens configuration, the task of finding all the images produced is in general a non-analytic problem. ${ }^{1}$ A multitude of mathematical tools have been brought to bear on this problem, such as complex analysis, Morse theory, resultants, and topology.

Starting the mathematical analysis of the problem, in Section 2.1, we review the basic set up of single-plane gravitational lensing and show the main results on the necessary and sufficient conditions for multiple images to be produced.

Following the multiple imaging results, in Section 2.2, we outline the main results that motivated the research presented in this thesis. Upon reviewing multiple papers in gravitational lensing we came across an article that described situations where the well known odd number theorem was not valid (see Gottlieb, 1994). This prompted a more detailed search in the literature and the discovery that the problem of the maximum

\footnotetext{
${ }^{1}$ The lens equation, when written in complex notation, is explicitly non-analytic as can be seen in equation (2.13).
} 
number of images was not yet solved.

As the simplest type of gravitational lens is the one represented by a point mass, bounds on the maximum number of images that a configuration of lenses can produce are easier to find for point-mass lenses. Then, extensions can be made for distributed masses. Following this idea, in Section 2.3 we review the literature available regarding bounds to the number of images for point-mass lenses.

Lastly, in Section 2.4 we review two recent results that provided the first answer to the question of the maximum number of images for a single plane configuration of point-mass lenses. First, a theorem in the area of complex analysis that when applied to the complex representation for the lens equation, provides an upper bound on the maximum number of images. The second result is a study into a set of configurations that proved to have exactly the number of images from the theorem on the upper bound, thus confirming that the upper bound is sharp and therefore it is the maximum number of images.

\subsection{Multiple Imaging}

In the previous chapter, equation (1.50) describes the basic lensing situation under study, where the lens distribution is thin and thus can be considered to lie on a plane between source and observer. Furthermore, this lens equation was derived in the weak field regime, where deflection angles for light rays are small and impact parameters on the lens plane are far from the Schwarzschild radius of masses in the deflector configuration. ${ }^{2}$

The lens equation (1.50) can be scaled to dimensionless quantities to simplify the study of its generic topological properties. If a length scale $\xi_{0}$ is set on the lens plane, then a corresponding length scale on the source place can be set as $\eta_{0}=\xi_{0} \frac{D_{O S}}{D_{O L}}$. This allows for the definition of dimensionless vectors for the positions of images and source on lens and source planes respectively. Setting $\mathbf{x}=\frac{\boldsymbol{\xi}}{\xi_{0}}$ as the dimensionless image position

\footnotetext{
${ }^{2}$ This excludes compact objects and black holes.
} 
on the lens plane and $\mathbf{y}=\frac{\boldsymbol{\eta}}{\eta_{0}}$ as the dimensionless source position on the source plane, equation (1.50) becomes

$$
\mathbf{y}=\mathbf{x}-\frac{D}{\xi_{0}} \boldsymbol{\alpha}\left(\xi_{0} \mathbf{x}\right)
$$

where the distance factor is defined as

$$
D \equiv \frac{D_{O L} D_{L S}}{D_{O S}}
$$

where the angular diameter distances from observer to lens plane, lens plane to source plane and observer to lens plane are $D_{O L}, D_{L S}$ and $D_{O S}$ respectively. Also, a scaled deflection angle can be defined as

$$
\hat{\boldsymbol{\alpha}}(\mathbf{x})=\frac{D}{\xi_{0}} \boldsymbol{\alpha}\left(\xi_{0} \mathbf{x}\right)
$$

so that the lens equation (2.1) further simplifies to

$$
\mathbf{y}=\mathbf{x}-\hat{\boldsymbol{\alpha}}(\mathbf{x})
$$

Now, a dimensionless surface mass density can be defined as $\kappa(\mathbf{x})=\sigma\left(\xi_{0} \mathbf{x}\right) / \sigma_{c r}$, where $\sigma$ is the surface mass density that results from projecting the lens mass onto the lens plane and $\sigma_{c r}$ is a critical surface mass density ${ }^{3}$ given by

$$
\sigma_{c r}=\frac{c^{2}}{4 \pi G D}
$$

Using equation (1.49) for the deflection angle, the scaled deflection angle defined in

\footnotetext{
${ }^{3}$ This density is critical in the sense that it separates the cases of strong lensing where multiple images are formed, from the cases of weak lensing where the effect of lensing is observed in the change of shape and position of the single image with respect to the unlensed source.
} 
equation (2.4) can be expressed in terms of $\kappa(\mathbf{x})$ as

$$
\hat{\boldsymbol{\alpha}}(\mathbf{x})=\frac{1}{\pi} \int_{\mathbb{R}^{2}} d^{2} x^{\prime} \kappa\left(\mathbf{x}^{\prime}\right) \frac{\mathbf{x}-\mathbf{x}^{\prime}}{\left|\mathbf{x}-\mathbf{x}^{\prime}\right|^{2}} .
$$

Following Schneider (1984), a scalar function $\phi(\mathbf{x}, \mathbf{y})$ can be defined by

$$
\phi(\mathbf{x}, \mathbf{y})=\frac{(\mathbf{x}-\mathbf{y})^{2}}{2}-\psi(\mathbf{x})
$$

where $\nabla \psi=\hat{\boldsymbol{\alpha}}$ is the scaled deflection angle (2.3), when written as a gradient of a scalar funcion $\psi(\mathbf{x})$ given by

$$
\psi(\mathbf{x})=\frac{1}{\pi} \int_{\mathbb{R}^{2}} d^{2} x^{\prime} \kappa\left(\mathbf{x}^{\prime}\right) \ln \left|\mathbf{x}-\mathbf{x}^{\prime}\right| .
$$

From equation (2.5), the lens equation (2.4) can be written in terms of $\phi$ as

$$
\nabla \phi(\mathbf{x}, \mathbf{y})=0
$$

where the gradient is taken with respect to $\mathbf{x}$.

Formulating gravitational lensing theory in terms of equation (2.7) is particularly insightful as then solutions that represent valid light rays and therefore real images are the critical points of a potential function. This is a clear statement of Fermat's Principle (see Schneider, 1985 and Blandford and Narayan, 1986) and the reason why the function $\phi$ defined in equation (2.5) is often called Fermat's Potential as it represents the the travel time for possible rays between source and observer.

Since images are the extrema of $\phi$ as given by equation (2.7), provided the Jacobian is not degenerate, it is possible to classify these extrema according to the determinant, trace and eigenvalues of the Jacobian matrix.

The magnification matrix ${ }^{4} \mathbf{J}=\frac{\partial \mathbf{y}}{\partial \mathbf{x}}$, with elements given by $J_{i j}=\frac{\partial y_{i}}{\partial x_{j}}$, denotes the

\footnotetext{
${ }^{4}$ It is called the magnification matrix because it contains the information on how the cross section
} 
Jacobian matrix of the transformation represented in the lens equation (2.4). Then, equations (2.5) and (2.7) imply that

$$
J_{i j}=\frac{\partial^{2} \phi}{\partial x_{j} \partial x_{i}}=\delta_{i j}-\frac{\partial^{2} \psi}{\partial x_{j} \partial x_{i}} .
$$

Since equation (2.6) implies that the two dimensional Laplacian of $\psi$ is given by $\triangle \psi=2 \kappa$ then the Jacobian matrix can be expressed as

$$
\mathbf{J}=\left(\begin{array}{cc}
1-\kappa-\gamma_{1} & -\gamma_{2} \\
-\gamma_{2} & 1-\kappa+\gamma_{1}
\end{array}\right)
$$

where

$$
\gamma_{1}=\frac{1}{2}\left(\frac{\partial^{2} \psi}{\partial x_{1}^{2}}-\frac{\partial^{2} \psi}{\partial x_{2}^{2}}\right) \quad \text { and } \quad \gamma_{2}=\frac{\partial^{2} \psi}{\partial x_{1} \partial x_{2}}=\frac{\partial^{2} \psi}{\partial x_{2} \partial x_{1}}
$$

Setting $\gamma=\left(\gamma_{1}^{2}+\gamma_{2}^{2}\right)^{1 / 2}$, the determinant, trace and eigenvalues of $\mathbf{J}$ are given by ${ }^{5}$

$$
\operatorname{det} \mathbf{J}=(1-\kappa)^{2}-\gamma^{2} \quad, \quad \operatorname{tr} \mathbf{J}=2(1-\kappa) \quad \text { and } \quad \lambda_{1,2}=1-\kappa \mp \gamma
$$

Images can then be classified according to the trace and the determinant of $\mathbf{J}$ as follows

$$
\begin{array}{rlc}
\operatorname{minimum} & \Leftrightarrow & \operatorname{det} \mathbf{J}>0, \operatorname{tr} \mathbf{J}>0 \\
\text { saddle-point } & \Leftrightarrow & \operatorname{det} \mathbf{J}<0 \\
\text { maximum } & \Leftrightarrow & \operatorname{det} \mathbf{J}>0, \operatorname{tr} \mathbf{J}<0
\end{array}
$$

Furthermore, as long as $\kappa(\mathbf{x})$ is smooth and goes to zero at infinity faster than $|\mathbf{x}|^{-2}$,

area of a light beam is distorted (see Section 1.5). The inverse of the absolute value of the determinant measures the flux amplification of an image over the flux of the unlensed source.

${ }^{5}$ Note that the determinant and eigenvalues of the magnification matrix are split into a term that depends on $\kappa$ called the convergence and a term that depends on $\gamma$ called the shear. The convergence represents a uniform scaling in the area of the light beam due to matter inside the beam (Ricci focusing), while the shear represents a distortion in the shape of the light beam due to matter outside the beam (Weyl focusing). For a discussion on Weyl and Ricci focusing in the context of gravitational lensing see Dyer and Roeder (1981a). 
equations (2.5) and (2.6) imply that $\phi$ increases quadratically for $|\mathbf{x}| \rightarrow \infty$ and thus $\phi$ has an absolute minimum, solution of $(2.7){ }^{6}$

Similarly, if the dimensionless surface mass density $\kappa(\mathbf{x})$ is smooth and tends to zero at infinity faster than $|\mathbf{x}|^{-2}$, a theorem on the conditions for a lens to produce multiple images can be formulated (see Subramanian and Cowling, 1986 or Section 5.4 in Schneider et al., 1992):

Theorem (Multiple Imaging). A gravitational lens produces multiple images if, and only if, there is a point $\mathbf{x}$ such that det $\mathbf{J}<0$. A sufficient but not necessary condition for multiple imaging is the existence of a point $\mathbf{x}$ where $\kappa(\mathbf{x})>1$.

The two statements in the theorem are easily proved. If $\operatorname{det} \mathbf{J}>0$ for all points $\mathbf{x}$ then the lens equation (2.4) is globally invertible and no multiple images are possible. Conversely, if there is a point $\mathbf{x}_{0}$ where $\operatorname{det} \mathbf{J}<0$, a source located at $\mathbf{y}\left(\mathbf{x}_{0}\right)$ produces and image that, according to the classification in (2.9), is a saddle-point of $\phi$. Since $\phi$ has also an absolute minimum, and thus another image, the lens produces multiple images.

In order to prove the statement on the necessary condition, note that if there is a point $\mathbf{x}_{0}$ with $\kappa\left(\mathbf{x}_{0}\right)>1$ then at that point $\operatorname{tr} \mathbf{J}<0$ (see equation 2.8). Therefore, a source located at $\mathbf{y}\left(\mathbf{x}_{0}\right)$ produces an image that according to (2.9) is either a saddle-point or a maximum of $\phi$. Since $\phi$ also has an image that is an absolute minimum, the lens produces multiple images.

The reasons for the condition that there exist some point $\mathbf{x}$ where $\kappa(\mathbf{x})>1$ is only a sufficient but not necessary condition for multiple imaging are explored by Subramanian and Cowling (1986), where it is found that symmetry in the lens plays a very important rôle in the production of multiple images.

Note that the preceding theorem and discussion on multiple imaging translate easily to the case of multiple lenses in a thin lens configuration as long as the bending for each of

\footnotetext{
${ }^{6}$ This does not apply to black holes or point-mass lenses as they are not smooth mass distributions.
} 
the lenses remains small and finite. In that case, the contributions to the bending angle from separate deflectors can be added and superimposed maintaining the conditions for the theorem.

An extension of results on the conditions for multiple imaging for thick lenses can be found in Padmanabhan and Subramanian (1988), where it is proved that a necessary and sufficient condition for multiple imaging is the existence of a conjugate point to the observer along a null geodesic located between observer and source. ${ }^{7}$

The case of a point-mass does not obey the postulate on the behaviour of the projected mass density as in that situation $\kappa(\mathbf{x})$ is not smooth. However, it is quite straightforward to show that such a lens will always produce multiple images. ${ }^{8}$ Recall that the bending angle for the point lens of mass $M$ is given by (see equation 1.42)

$$
\alpha=\frac{4 G M}{c^{2} \xi},
$$

where $\xi$ is the impact parameter on the lens plane. ${ }^{9}$ Thanks to the symmetry, the problem reduces to just one dimension and all vector quantities can be replaced by scalars. In order to write the scaled dimensionless lens equation, $\xi_{0}$ can be set as $\xi_{0}=R_{E}$, where

$$
R_{E} \equiv \sqrt{\frac{4 G M}{c^{2}} D}
$$

and $D$ is the distance factor defined in (2.2). The scale $R_{E}$ is called the Einstein Radius of mass $M$, because a source perfectly aligned with a foreground deflecting mass will be imaged as a ring of radius $R_{E}$ around the lens. ${ }^{10}$ The scaled deflection angle in terms of

\footnotetext{
${ }^{7}$ Two points $\mathrm{P}$ and $\mathrm{Q}$ that lie along a null geodesic are called conjugate if the area of a light beam vanishes at both points but the derivative of the area of the light beam with respect to an affine parameter has opposite signs. For a more rigorous definition see Section 9.3 in Wald (1984).

${ }^{8}$ Furthermore, the study of point-mass lenses is rather useful since the exterior of any spherically symmetric mass distribution is described by the Schwarzschild metric used to derive the bending angle in the linearized weak field regime.

${ }^{9}$ The impact parameter $\xi$ is much larger than the Schwarzschild radius $R_{S}=\frac{2 G M}{c^{2}}$ in order to remain in the linearized approximation to the Schwarzschild metric used to derive (2.10).

${ }^{10}$ In a published note Chwolson (1924) describes this ring effect, however Renn et al. (1997) discovered
} 
(2.11) simplifies to (see equation 2.3)

$$
\hat{\alpha}=\frac{1}{x}
$$

and the scaled dimensionless lens equation (2.4) becomes

$$
y=x-\frac{1}{x}
$$

Given a position $y$ for the source, images will then be observed at positions $x$ that are solutions of (2.12). The two solutions are given by

$$
x_{ \pm}=\frac{y \pm \sqrt{y^{2}+4}}{2}
$$

so that a point-mass always produces multiple images.

\subsection{The Odd Number Theorem}

Having established that, due to the non-analyticity of the lens equation, gravitational lensing can produce multiple images, studies turned to the search for results in image counting and general properties of the number of images that given distributions can produce. One of the first generic results in the study of the number of images is known as the Odd Number Theorem. Briefly stated, a gravitational lens always produces an odd number of images of a distant source. ${ }^{11}$

Initially, the theorem was proved for spherical mass distributions using basic calculus in Dyer and Roeder (1980). A basic assumption for the bending approximation was used so that, as long as the projected mass density diverges slower than $|\mathbf{x}|^{-1}$ as $\mathbf{x}$ approaches

unpublished notes by Einstein on this effect dated back to 1912 .

${ }^{11}$ Given the conditions on the mass distribution for the validity of the theorem, point-mass lenses are excluded. 
zero at the center of the lens, then the theorem is valid (see for example Clark, 1972).

A generalization for non-spherical mass distributions was proved in Burke (1981), where it is still required that the bending remains finite, a consequence of the bounding condition imposed on the projected mass density. The main tool used to prove this instance is the Poincaré-Hopf Index Theorem (see Guillemin and Pollack, 1974), which can be seen as a natural generalization of the calculus arguments employed in the first proof in Dyer and Roeder (1980).

Except for the calculation of bending in the linearized regime near the deflecting mass, the results in Dyer and Roeder (1980) and Burke (1981) require Euclidean geometry, an approximation that is very realistic for most cases of gravitational lensing. However, in order to extend the theorem for a larger set of manifolds that General Relativity relativity is built on, a proof using Uhlenbeck's Morse theory (see Uhlenbeck, 1975) is presented in McKenzie (1985).

Two examples of exotic space-times where the odd number theorem does not apply are shown in Gottlieb (1994), where necessary and sufficient conditions on the manifolds are shown. Explicitly, a space-time leads to gravitational lensing where the odd number theorem is valid, as long as bundles of null geodesics intersect space-like slices in twospheres. A list of other space-times where the theorem is not valid can be constructed using warped products as described in O'Neill (1983).

A restatement of the theorem using Morse theory can be found in Petters (1992) and in Petters et al. (2001), where Morse inequalities (see Milnor, 1963) are applied to the time delay function that describes gravitational lensing for generic mass distributions.

The combination and interplay between mathematics and physics that is evident in the evolution of the odd number theorem in gravitational lensing motivated and prompted the question of the maximum number of images. Even though there were numerous results regarding the odd number theorem and a variety of similar results for the conditions on multiple imaging and minimum number of images, the results for a maximum were very 
scarce and imprecise.

Furthermore, emboldened by a maxim often heard and quoted in textbooks for several courses in mathematics, we decided to study the problem of maximal lensing in image counting: What is the maximum number of images that a gravitational lens can produce? The maxim is: Never underestimate the power of a theorem that counts something (see for example the various comments on the theorems of Lagrange and Sylow in Fraleigh, 1967).

\subsection{Bounds on the Number of Images}

Given its relative simplicity, the point-mass lens makes for the ideal candidate to study multiple imaging in gravitational lensing. Written in complex notation (see Bourassa et al., 1973 and Bourassa and Kantowski, 1975) and normalized (see Section 5.1 in Schneider et al., 1992), the lens equation for $k=1, \ldots, n$ point lenses of mass $m_{k}$ takes the form

$$
z_{s}=z-\sum_{k=1}^{n} \frac{m_{k}}{\bar{z}-\bar{z}_{k}}
$$

where $z_{s}$ is the position of the source, $z_{k}$ denotes the position of the each point-mass, the bar denotes complex conjugation and the equation is to be solved for $z$ that represents the positions of images formed by the gravitational lens.

A lower bound on the number of images that a configuration described by equation (2.13) can produce was found using Morse theory (see Petters, 1992, 1995a and Section 11.4 in Petters et al., 2001) or alternatively using resultants (see Section 11.5.2 in Petters et al., 2001 and Petters, 1997), so that the number of images produced is not less than $n-1$. Furthermore, a similar approach using resultants or Morse theory can be applied to the case of multiplane gravitational lensing, where the point masses are not all at the same distance from the observer. In this multiplane configuration, for $n$ point-mass lenses located at $n$ different distances from the observer, a lower bound of $2^{n}$ images can 
be shown (see Petters, 1995a, 1996, 1997). Additionally, in Petters (1996) it is shown that both, the lower bound of $2^{n}$ for the multiplane configurations and the lower bound of $n-1$ for the single plane configurations, can be achieved and thus they represent the minimum number of images for the respective scenarios.

Using also resultants (see Section 11.5.2 in Petters et al., 2001 and Petters, 1997), an upper bound on the number of images was found to be $n^{2}+1$ for the single plane case, and $2\left(2^{2(n-1)}-1\right)$ for the multiplane case (see Section 12.3 in Petters et al., 2001).

An alternative way to establish the upper bound of $n^{2}+1$ images for the single-plane configuration of $n$ point-mass lenses can be found in Witt (1990), where the lens equation (2.13) is transformed into a complex polynomial of degree $n^{2}+1$. Then, by showing that all solutions of the lens equation are solutions of the polynomial it is proved that $n^{2}+1$, being the number of solutions of the polynomial, is thus an upper bound for the number of images.

However, given that the lens equation is not analytic and the polynomial is clearly analytic (see Rhie, 2002), it is important to note that not all solutions of the polynomial are images and therefore, it is not possible to conclude that $n^{2}+1$ is the maximum number of images. An example of this erroneous line of thought can be found in Rhie (1997). Furthermore, configurations like those illustrated in Figures 5.11, 5.12 and 5.13, show the differences between solutions of the polynomial (indicated by small squares) and solutions of the lens equation (indicated by stars). The details regarding these graphs and the relevance of the corresponding lens configurations to the study of maximal lensing can be found in Section 5.3 and Section B.3.

In order to further study the production of images and maximal lensing, the polynomial approach in finding the upper bound is more efficient than the result derived from resultants because the polynomial provides with a tool to calculate and find the images directly where the use of resultants. To find images one can then set up the polynomial, find all the solutions and then feed the solutions into the lens equation to separate real 
images from those that are a product of the transformation from lens equation to polynomial representation. This approach was used to obtain the results found in Chapter 5 and is the basis of the code implemented and detailed for the various cases in the Appendix B. Given the extensive use of the polynomial representation in the following chapters, following Witt (1990) and Rhie (2002) we reproduce the derivation.

Given that the lens equation (2.13) is linear in $z$, it is possible to embed it into an analytic equation. That is, it is possible to find an analytic equation such that all solutions of the lens equation are also solutions of this analytic equation. Following Rhie (2002), set

$$
f\left(z ; z_{k}\right) \equiv \sum_{k=1}^{n} \frac{m_{k}}{z-z_{k}}
$$

so that $z_{s}=z-f\left(\bar{z} ; \bar{z}_{k}\right)$ and $\bar{z}_{s}=\bar{z}-f\left(z ; z_{k}\right)$. This manipulation allows for equation (2.13) to be rewritten as:

$$
z_{s}=z-f\left(f\left(z ; z_{k}\right)+\bar{z}_{s} ; \bar{z}_{k}\right)
$$

It is then straightforward to verify that if $z$ is a solution of (2.13) then it is also a solution of (2.14). Thus, the non-analytic lens equation is embedded into an analytic expression.

Now, following Witt (1990), the embedding can be made explicit to obtain an expression that allows for solutions to be found in an easier way than through the lens equation. Taking the complex conjugate of (2.13) and multiplying this by $\prod_{j=1}^{n}\left(z_{j}-z\right)$ one obtains

$$
\left(\bar{z}_{s}-\bar{z}\right) \prod_{j=1}^{n}\left(z_{j}-z\right)=\sum_{i=1}^{n} m_{i} \prod_{\substack{j=1 \\ j \neq i}}^{n}\left(z_{j}-z\right)
$$

Solving for $z$ from equation (2.13), it follows that

$$
z=z_{s}-\sum_{k=1}^{n} \frac{m_{k}}{\bar{z}_{k}-\bar{z}}
$$


Replacing the value of $z$ from (2.16) into (2.15), the resulting equation depends explicitly only on $\bar{z}$ as follows

$$
\left(\bar{z}_{s}-\bar{z}\right) \prod_{j=1}^{n}\left(z_{j}-z_{s}+\sum_{k=1}^{n} \frac{m_{k}}{\bar{z}_{k}-\bar{z}}\right)=\sum_{i=1}^{n} m_{i} \prod_{\substack{j=1 \\ j \neq i}}^{n}\left(z_{j}-z_{s}+\sum_{k=1}^{n} \frac{m_{k}}{\bar{z}_{k}-\bar{z}}\right) .
$$

In order to eliminate denominators, equation (2.17) can be multiplied by

$$
\left[\prod_{k=1}^{n}\left(\bar{z}_{k}-\bar{z}\right)\right]^{n}
$$

resulting in a polynomial in $\bar{z}$, that when complex conjugated is a polynomial in $z$ alone

$$
\begin{aligned}
& \left(z-z_{s}\right) \prod_{j=1}^{n}\left(\left(\bar{z}_{j}-\bar{z}_{s}\right) \prod_{k=1}^{n}\left(z_{k}-z\right)+\sum_{k=1}^{n} m_{k} \prod_{\substack{l=1 \\
l \neq k}}^{n}\left(z_{l}-z\right)\right)+ \\
& \prod_{k=1}^{n}\left(z_{k}-z\right) \sum_{i=1}^{n} m_{i} \prod_{\substack{j=1 \\
j \neq i}}^{n}\left(\left(\bar{z}_{j}-\bar{z}_{s}\right) \prod_{k=1}^{n}\left(z_{k}-z\right)+\sum_{k=1}^{n} m_{k} \prod_{\substack{l=1 \\
l \neq k}}^{n}\left(z_{l}-z\right)\right)=0 .
\end{aligned}
$$

Equation (2.18) being a polynomial that depends explicitly only on $z$, is analytic and therefore makes explicit the embedding previously mentioned. The degree of the polynomial (2.18) is $n^{2}+1$ and therefore it has that many roots, setting an upper bound to the number of images. This upper bound is not necessarily a maximum as the embedding into the analytic polynomial guarantees only that solutions of the lens equation are solutions of the polynomial but not the other way around. In other words, some zeros of the polynomial (2.18) do not satisfy the lens equation (2.13) and therefore they do not represent images.

For the special case of $n$ identical masses located at the vertices of an equilateral polygon centered at the projected position of the source on the lens plane, in Mao et al. (1999) it is shown that the lens equation can be solved analytically. Given that a similar procedure will be used in Chapter 3, here we reproduce this result. Using complex polar 
notation, let $z=\rho e^{i \theta}$ for the image position and $z_{s}=0$ for the position of the source in equation (2.13). Furthermore, the lenses at the vertices of the regular polygon have positions given by $z_{k}=r e^{i 2 \pi k / n}$, where $r$ is the radius of the polygon and $k=1, \ldots, n$. Thus, in polar coordinates equation (2.13) becomes

$$
\rho=\frac{\rho^{n-1}}{\rho^{n}-r^{n} e^{i n \theta}}
$$

where since $r$ and $\rho$ are real, $e^{i n \theta}$ must be real. Therefore, $e^{i n \theta}= \pm 1$, and equation (2.19) simplifies to

$$
\rho^{n}-\rho^{n-2} \mp r^{n}=0 \text {. }
$$

Using Descartes's rule of signs and basic calculus on equation (2.20), it is then straightforward to show that the number of solutions and thus, the number of images is 5 for $n=2$ and $3 n+1$ for $n>2$, as long as $r$ is less than a critical value $r_{c r}$ given by

$$
r_{c r}=\left(\left[\frac{n-2}{n}\right]^{(n-2) / 2}-\left[\frac{n-2}{n}\right]^{n / 2}\right)^{1 / n} .
$$

This result gives a lower bound on the maximum number of images, so that if $N_{\max }$ denotes the maximum number of images for a configuration of $n$ point-mass lenses, then

$$
3 n+1 \leq N_{\max } \leq n^{2}+1
$$

A study of configurations with $n=4$ point-mass lenses in Rhie (2001) revealed that it was possible to obtain 15 images, that is, two more than the $3 n+1=13$ present in the perfectly symmetric case. This was achieved by perturbing the positions of the lenses away from the square configuration. However, in this same paper it is proved that $n^{2}+1=17$ images cannot be produced. This means that the maximum number of images is strictly larger than the one produced by a perfectly symmetric configuration as 
described in (2.20) and it is also strictly smaller than the maximum number of solutions found in the polynomial representation (2.18). Thus, if $N_{\max }$ is the maximum number of images for a system of $n$ point-mass lenses on a plane, then

$$
3 n+1<N_{\max }<n^{2}+1
$$

Furthermore, the increase in the number of images through a perturbation on the symmetric case led the author to suggest that the maximum number of images is produced when the configuration is symmetric enough to maximize the gravitational interference of the deflecting masses but not so symmetric that images merge due to degenerate critical points (see Rhie, 2001).

\subsection{A Theorem in Complex Analysis}

In a recent paper on the field of complex analysis for rational harmonic functions, the following result is proved (see Khavinson and Neumann, 2006):

Theorem (Khavinson-Neumann). Let $r(z)=\frac{p(z)}{q(z)}$ be a rational function where $p$ and $q$ are relatively prime, analytic polynomials and $n=\operatorname{deg}(r(z))=\max (\operatorname{deg} p, \operatorname{deg} q)$. If $n>1$, then $f(z)=\overline{r(z)}-z$ has at most $5 n-5$ distinct finite zeros.

By setting $r(z)=\bar{z}_{s}+\sum_{k=1}^{n} \frac{m_{k}}{z-z_{k}}$, the lens equation in the point-mass case described by (2.13) can be written as $\overline{r(z)}=z$ and therefore the theorem can be applied. This implies that the maximum number of images $N_{\max }$ cannot be $n^{2}+1$ and relationship (2.21) must be corrected to

$$
3 n+1<N_{\max } \leq 5 n-5
$$

In a study of the properties of the critical curves of the point mass configuration (see Rhie, 2003), a special arrangement of $n$ point lenses producing a total of $5 n-5$ images 
was found. This configuration consists of $n-1$ equal masses located at the vertices of a regular polygon with $n-1$ sides, along with a perturbation in the form of a small mass located at the center of the polygon. The introduction of the small mass at the center causes the number of images to go from $3(n-1)+1$ to $5 n-5$. This last result implies that $5 n-5$ is the maximum number of images for a configuration of $n$ point-mass lenses. However, no explicit bounds are established for the central mass that leads to the maximal lensing case.

In Chapter 3 we give an alternate proof that $5 n-5$ is the maximum number of images while providing more details on the maximal lensing configuration. That is, we calculate an explicit bound on the central mass that leads to the maximal lensing case. An early version of this result was recently published (see Bayer and Dyer, 2007).

In Chapter 4 we extend the results from point mass lenses to distributed masses to show that the effect of replacing a point lens with a distributed mass is the increase in the number of images by one. Therefore, a system of $n$ distributed lenses produces a maximum of $6(n-1)+1$ images. This last result can also be found in Bayer et al. (2006).

It is worth noting that for the case of multiplane gravitational lensing, no further results have been found beyond the lower and upper bounds found through Morse theory and resultants (see Petters, 1997 and Theorem 12.10 in Petters et al., 2001). 


\section{Chapter 3}

\section{Symmetric Point-Mass Configurations}

In Chapter 2 we reviewed existing results that lead to the description of a maximal lensing configuration as well as a proof that the maximum number of images produced by a configuration of $n$ point-mass lenses is $5 n-5$. This result is a combination of a theorem on complex rational functions presented by Khavinson and Neumann (2006) with the specific lens construction made by Rhie (2003) for $n-1$ point-mass lenses at the vertices of a regular polygon and a small mass lens at the center of the polygon.

However, the understanding of maximal lensing for point-mass lenses remains incom-

plete since the result obtained by Rhie (2003) provides no specific bounds or constraints on the mass that must be placed at the center of the configuration in order to produce the maximum number of images.

In this chapter, we present a simpler proof that the configuration of point lenses in a regular polygon with a small central mass is a maximal lensing case, while extending the previous result by the calculation of an explicit upper bound for the central mass perturbation. 


\subsection{Central Mass for Maximal Lensing}

For $n \geq 4$, consider a total of $n-1$ equal point masses. In units of the total mass $M$, each one of the point masses will be $m_{k}=\frac{1}{n-1}$ so that

$$
\sum_{k=1}^{n-1} m_{k}=1
$$

These masses will be located at the vertices of a regular polygon of $n-1$ sides. The radius $r$ of the polygon is measured in units of $R_{E}$, where $R_{E}$ is the Einstein radius of the total mass $M$ of the lenses at the vertices of the polygon. Thus, according to equation (2.11), $R_{E}=\sqrt{D \Phi M}$, where $\Phi=\frac{4 G}{c^{2}}$ and $D$ is the distance factor defined in $(2.2)$.

The position of the $n-1$ point masses can be represented in complex polar form by $z_{k}=r e^{i 2 \pi k / n-1}$, for $k=0, \ldots, n-2$. In order for this symmetric configuration to produce $3(n-1)+1$ images, the radius $r$ of the polygon must be less than a critical value $r_{c r}$ given by Mao et al. (1999) as

$$
r_{c r}=\left(\left[\frac{n-3}{n-1}\right]^{(n-3) / 2}-\left[\frac{n-3}{n-1}\right]^{(n-1) / 2}\right)^{1 /(n-1)}
$$

Illustrated in Figure 3.1 is a symmetric lensing configuration for 5 equal point masses, each with $m=\frac{1}{5}$, arranged in a regular pentagon with the source at $z_{s}=0$. The behaviour of $r_{c r}$ as a function of the number of lenses in the polygon is illustrated in Figure 3.2, and it can be verified that (see Section A.1)

$$
\frac{1}{\sqrt{2}} \leq r_{c r}<1
$$

so from this point onwards we will consider that $r<\frac{1}{\sqrt{2}}$ for all values of $n \geq 4$.

The perturbation is specified by locating a lens of mass $m$ at the center of the polygon, where $m$ is measured in the same units as $m_{k}$. In complex form, the normalized lens 


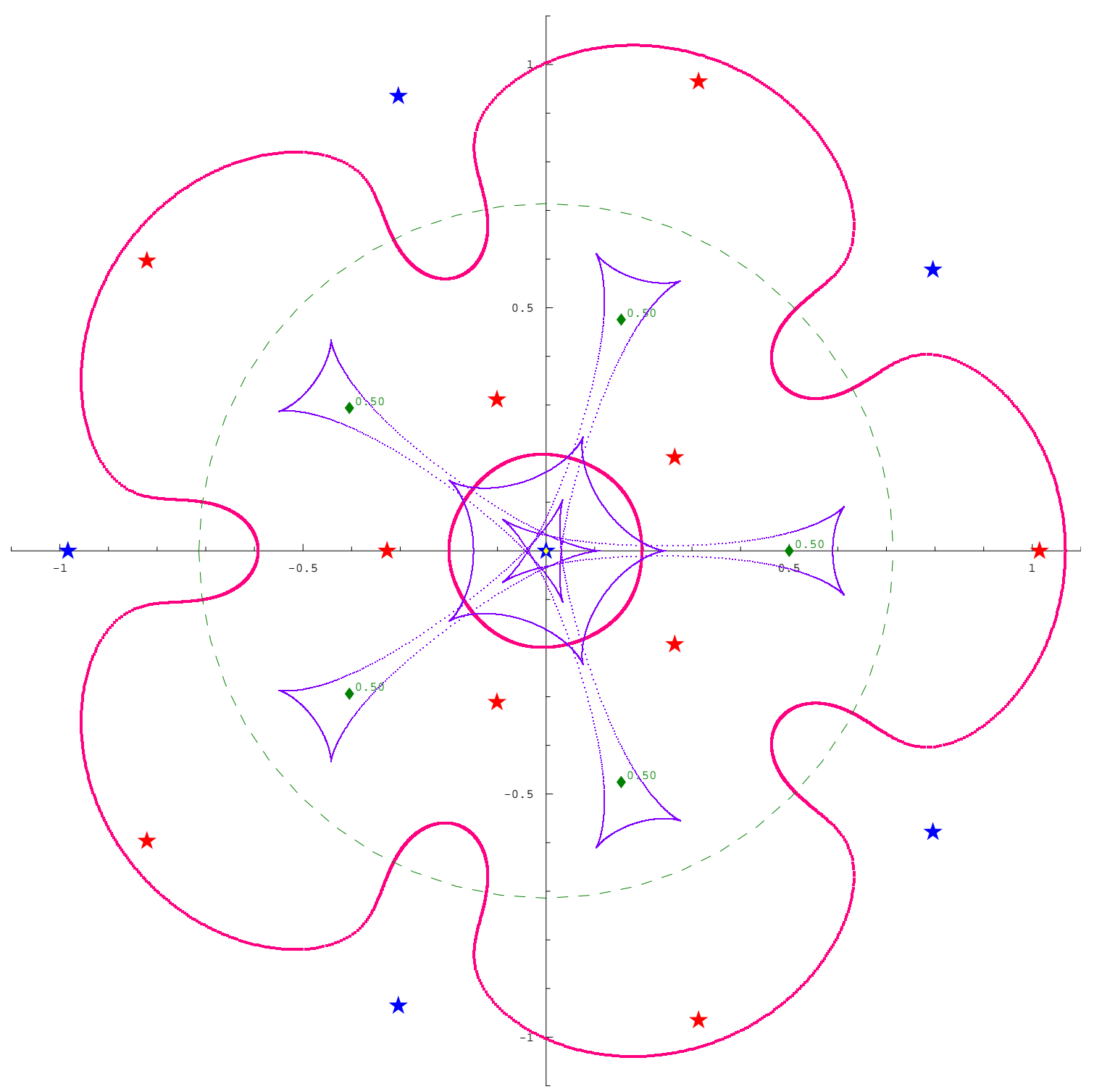

Figure 3.1: Symmetric point-mass lensing configuration for $n=5$ lenses in a regular pentagon. The dashed line represents the circle of radius $r_{c r} \cong 0.71$. Since the lenses, indicated with diamonds, are located at $r=0.50<r_{c r}$, the number of images produced is $N=16$. Images are represented by five-pointed stars, while thick smooth lines represent critical curves and thin cuspy lines represent the caustic curves. 


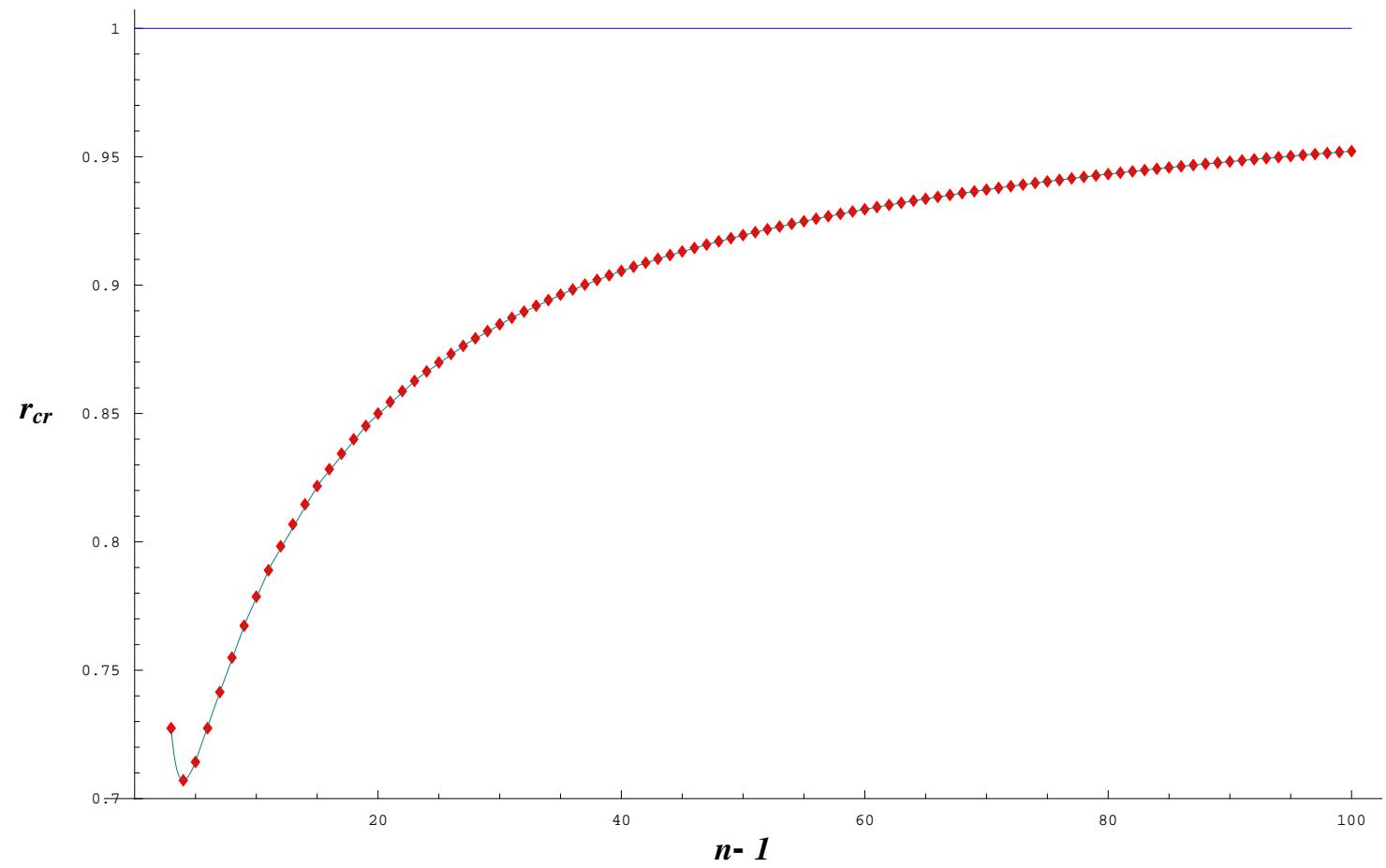

Figure 3.2: The critical radius $r_{c r}$, in units of the Einstein radius, as a function of the number of lenses $n-1$ located at the vertices of a regular $(n-1)$-polygon. Note the asymptotic increase to $r=1$ in the interval $[4, \infty)$ and the minimum at $n-1=4$.

equation can thus be written for the $n$ point masses:

$$
z_{s}=z-\sum_{k=1}^{n-1} \frac{m_{k}}{\bar{z}-\bar{z}_{k}}-\frac{m}{\bar{z}}
$$

where $z_{s}$ is the position of the source when projected on the lens plane. An example of this type of configuration can be constructed by the addition of a perturbation in the form of a central mass $m=\frac{1}{50}$ to the previous example illustrated in Figure 3.1. The perturbed lensing configuration illustrating equation (3.3) can be found in Figure 3.3. Simplifying the sum in equation (3.3) the lens equation becomes

$$
z_{s}=z-\frac{\bar{z}^{n-2}}{\bar{z}^{n-1}-r^{n-1}}-\frac{m}{\bar{z}}
$$

Locating the source at the center of symmetry so that $z_{s}=0$, and writing $z$ in polar 


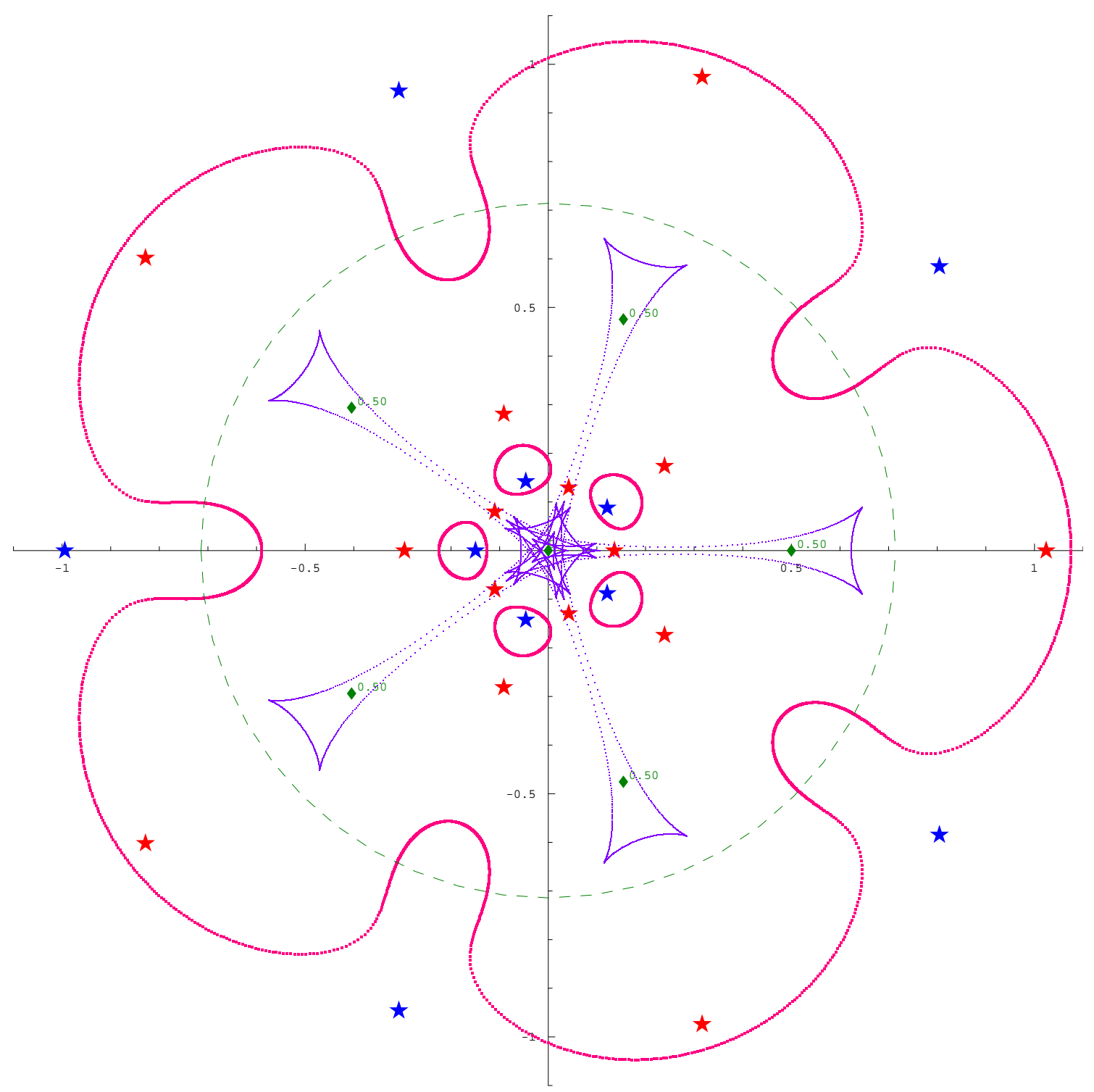

Figure 3.3: Central mass perturbation of $m=\frac{1}{50}$ on a symmetric point-mass lensing configuration of $n=5$ lenses in a regular pentagon. The dashed line represents the circle of radius $r_{c r} \cong 0.71$. Lenses at the vertices of the pentagon, indicated with diamonds, are located at $r=0.50<r_{c r}$. The small mass perturbation at the center of the configuration increases the number of images to $N=25$. Images are represented by five-pointed stars, while thick smooth lines represent critical curves and thin cuspy lines represent the caustic curves. 
form as $z=\rho e^{i \theta}$, where $\rho$ and $\theta$ are real, equation (3.4) simplifies to

$$
\rho^{2}=\frac{\rho^{n-1}}{\rho^{n-1}-r^{n-1} e^{i(n-1) \theta}}+m
$$

Given that $\rho, r$ and $m$ are real, it follows from equation (3.5) that $e^{i(n-1) \theta}$ must be real. Thus, the only possible values are $e^{i(n-1) \theta}= \pm 1$. For $e^{i(n-1) \theta}=+1$, equation (3.5) can be written as $f_{+}(\rho)=0$ by defining

$$
f_{+}(\rho)=\rho^{n+1}-(1+m) \rho^{n-1}-r^{n-1} \rho^{2}+m r^{n-1} .
$$

Similarly, for $e^{i(n-1) \theta}=-1$, equation (3.5) can be written as $f_{-}(\rho)=0$ by defining

$$
f_{-}(\rho)=\rho^{n+1}-(1+m) \rho^{n-1}+r^{n-1} \rho^{2}-m r^{n-1} .
$$

Counting the sign changes of the coefficients in equation (3.6), Descartes's rule of signs implies that $n_{+}$, the number of positive roots of $f_{+}(\rho)=0$, is either $n_{+}=0$ or $n_{+}=2$. The same argument applied to equation (3.7) implies that $n_{-}$, the number of positive roots of $f_{-}(\rho)=0$, is either $n_{-}=1$ or $n_{-}=3$. Thus, the total number of positive roots of $f_{ \pm}(\rho)=0$ can be no greater than 5 .

Since $e^{i(n-1) \theta}=+1$ has $n-1$ solutions, each positive root of $f_{+}(\rho)=0$ corresponds to $n-1$ solutions and consequently $n-1$ images for the lens equation (3.4). Similarly, since $e^{i(n-1) \theta}=-1$ has $n-1$ solutions, each positive root of $f_{-}(\rho)=0$ corresponds to $n-1$ images. Given that the number of positive roots of $f_{ \pm}(\rho)=0$ is not greater than 5 , the total number of solutions and images for the lens equation (3.3) can be no greater than $5(n-1)$.

To show that $n_{+}=2$ is always realized, first note that $f_{+}(0)=m r^{n-1}$ is positive. Furthermore, $f_{+}(r)=-r^{n-1}$ is negative and $f_{+}(\rho)$ is positive for large values of $\rho$. Therefore, there exist $\rho_{1}$ and $\rho_{2}$ such that $0<\rho_{1}<r<\rho_{2}$ and $f_{+}\left(\rho_{1}\right)=f_{+}\left(\rho_{2}\right)=0$, so 


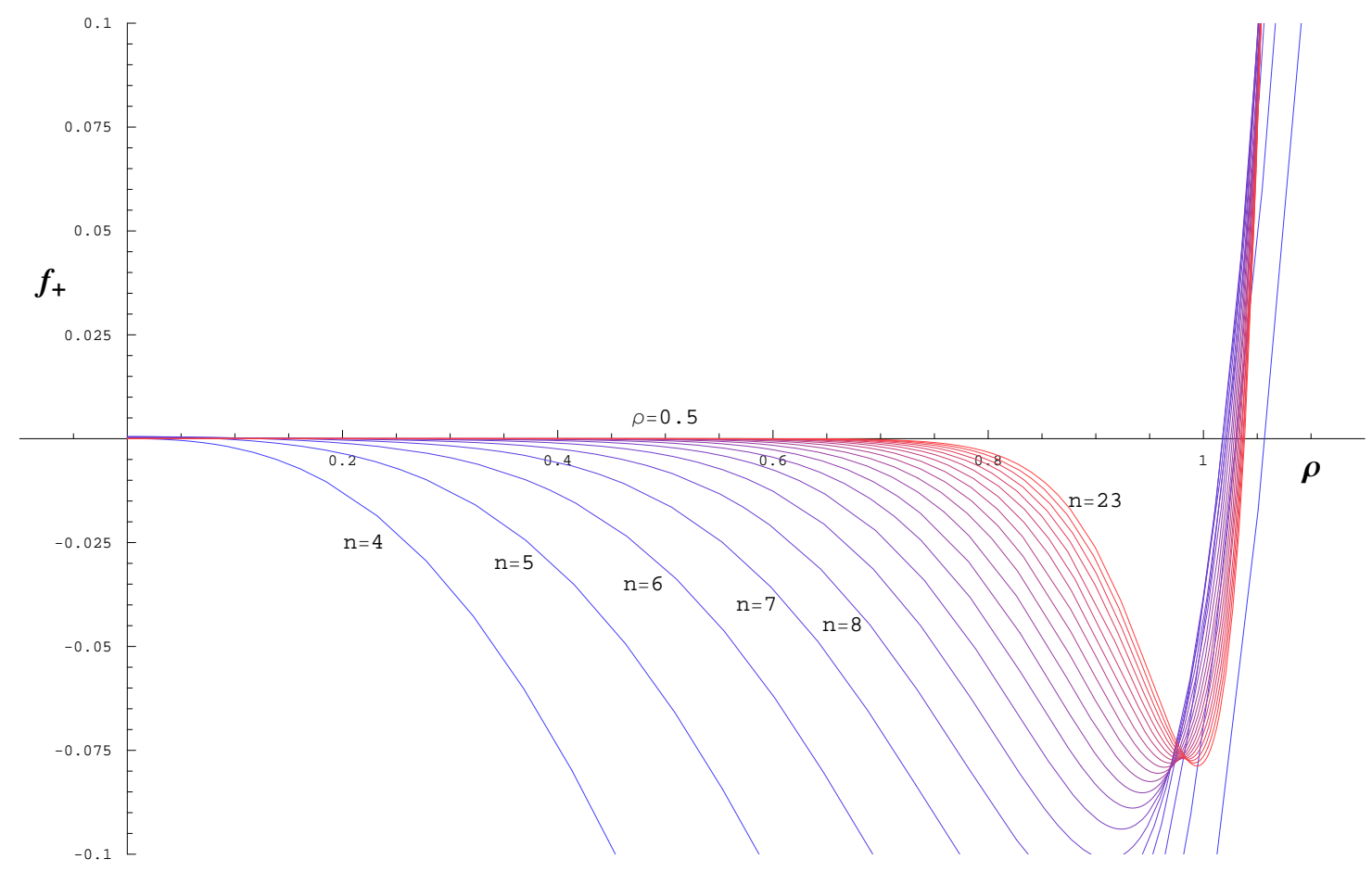

Figure 3.4: Graphs for $f_{+}(\rho)$ for various cases of $n$ and $r=\frac{1}{2}$. Note that for large values of $\rho$ all the functions are positive and that at $\rho=r=\frac{1}{2}$ all functions are negative. The mass is $m_{\star}\left(r=\frac{1}{2}\right) / 2$ and $\rho$ is given in terms of the Einstein radius.

that $n_{+}=2$. Graphically, the behaviour of $f_{+}(\rho)$ is illustrated for $r=\frac{1}{2}$ in Figures 3.4 and 3.5.

To show that $n_{-}=3$ can be realized, conditions must be imposed on the central mass perturbation. First, note that $f_{-}(0)=-m r^{n-1}$ is negative and $f_{-}(\rho)$ is positive for large values of $\rho$. Furthermore, since $r<\frac{1}{\sqrt{2}}$, it follows that

$$
f_{-}(r)=r^{n-1}\left(2 r^{2}-2 m-1\right)
$$

is negative.

One last condition for $n_{-}=3$ depends on a careful choice of $\rho$ between 0 and $r$, such that $f_{-}(\rho)>0$. Since $r<\frac{1}{\sqrt{2}}$, a suitable choice for $\rho$ that lies between 0 and $r$ is

$$
\rho_{0}=r^{1+\frac{6}{n-1}}
$$




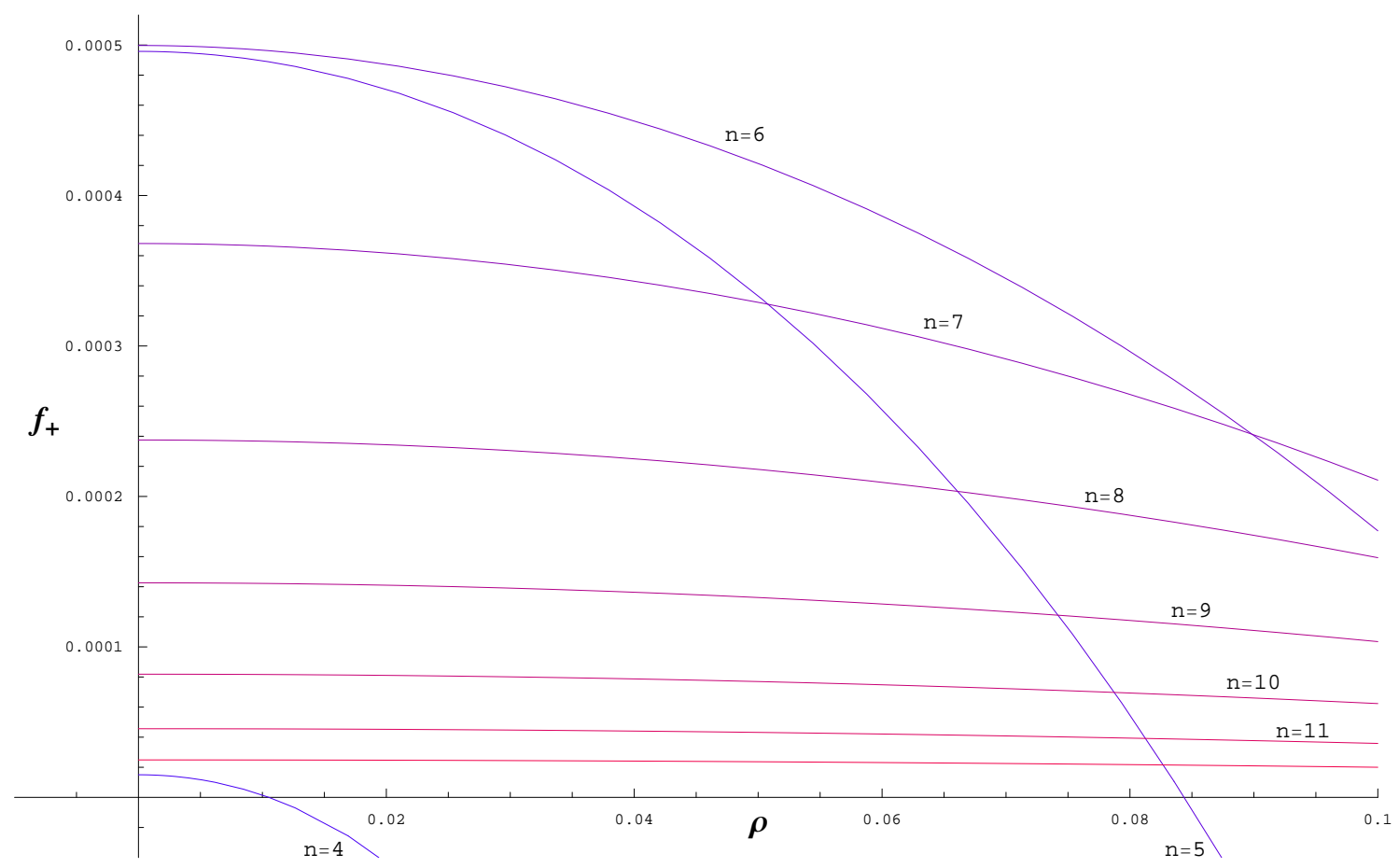

Figure 3.5: Graphs for $f_{+}(\rho)$ for various cases of $n$ and $r=\frac{1}{2}$. Note that at $\rho=0$ all functions are positive. The mass is $m_{\star}\left(r=\frac{1}{2}\right) / 2$ and $\rho$ is given in terms of the Einstein radius.

Furthermore, since

$$
f_{-}\left(\rho_{0}\right)=r^{n-1}\left\{r^{2\left(\frac{n+5}{n-1}\right)}\left(1+r^{6}\right)-m\left(1+r^{6}\right)-r^{6}\right\}
$$

by imposing the condition $f_{-}\left(\rho_{0}\right)>0$, we obtain an upper bound $m_{\star}$ for $m$, given by

$$
m_{\star}=r^{2\left(\frac{n+5}{n-1}\right)}-\frac{r^{6}}{1+r^{6}}
$$

Note that for $r<1$ and $n \geq 4$

$$
r^{2\left(\frac{n+5}{n-1}\right)} \geq r^{6}
$$

and since

$$
r^{2\left(\frac{n+5}{n-1}\right)}\left(1+r^{6}\right)>r^{2\left(\frac{n+5}{n-1}\right)}
$$

it is then guaranteed that $m_{\star}>0$. 


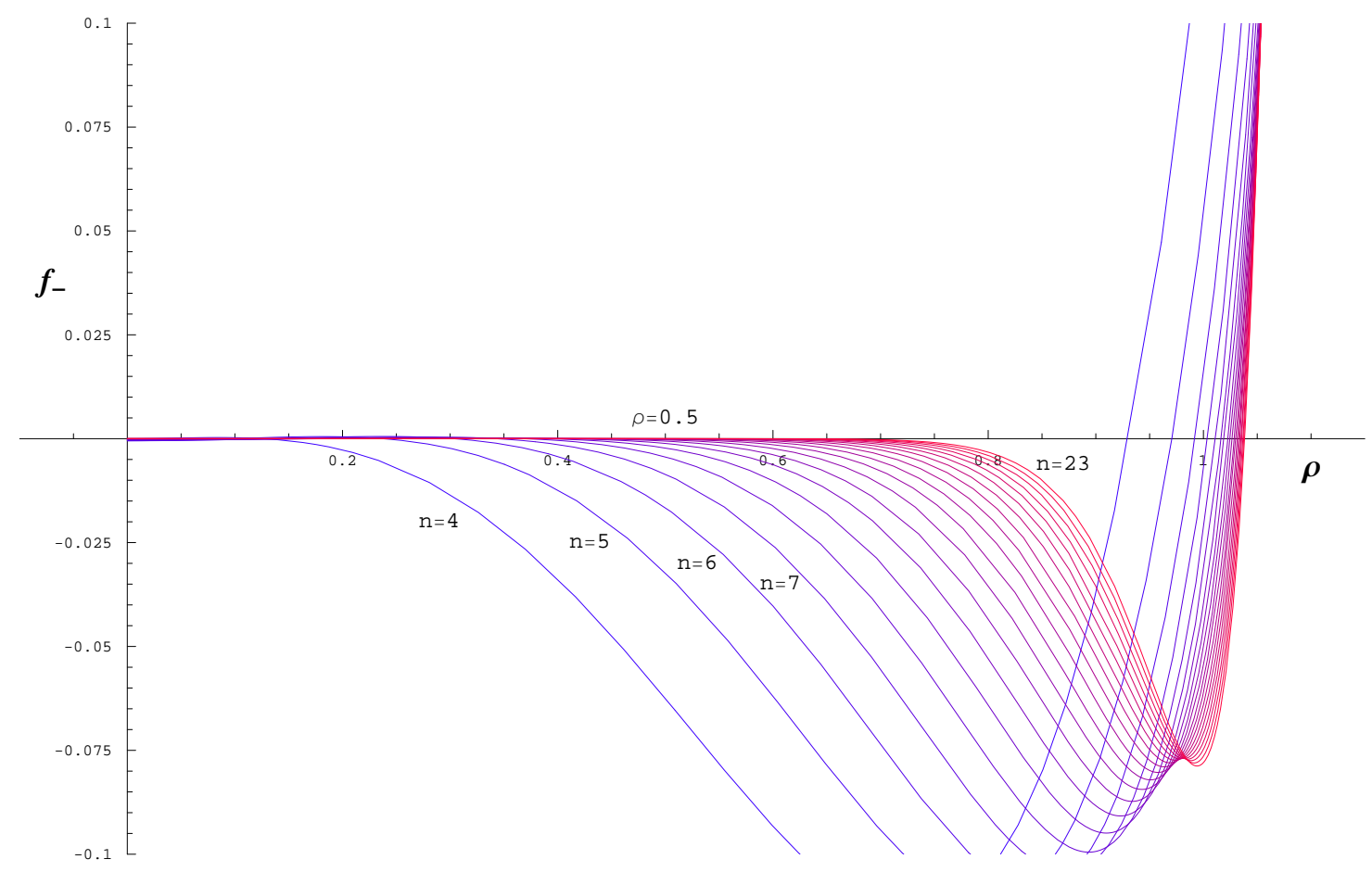

Figure 3.6: Graphs for $f_{-}(\rho)$ for various cases of $n$ and $r=\frac{1}{2}$. Note that for large values of $\rho$ all the functions are positive and that at $\rho=r=\frac{1}{2}$ all functions are negative. The mass is $m_{\star}\left(r=\frac{1}{2}\right) / 2$ and $\rho$ is given in terms of the Einstein radius.

Given the value of $f_{-}(\rho)$ for large values of $\rho$, and the values found for $f_{-}(0), f_{-}\left(\rho_{0}\right)$ and $f_{-}(r)$ it follows that $f_{-}(\rho)=0$ has 3 different positive solutions, provided that the central mass perturbation $m$ remains bounded above by $m_{\star}$ given by (3.9). Thus, $n_{-}=3$ is realizable for the lens configuration under study. Graphically, the behaviour of $f_{-}(\rho)$ is illustrated for $r=\frac{1}{2}$ in Figures 3.6 and 3.7.

We have shown that $n_{+}=2$ is always realized and determined conditions under which $n_{-}=3$ can be realized. It then follows that a system of $n$ point lenses can produce a total of $5 n-5$ images of a distant source. Furthermore, since the main theorem in Khavinson and Neumann (2006) referenced in Section 2.4 proves that $5 n-5$ is an upper bound, we conclude that this upper bound is the maximum number of images for a system of $n$ point lenses. 


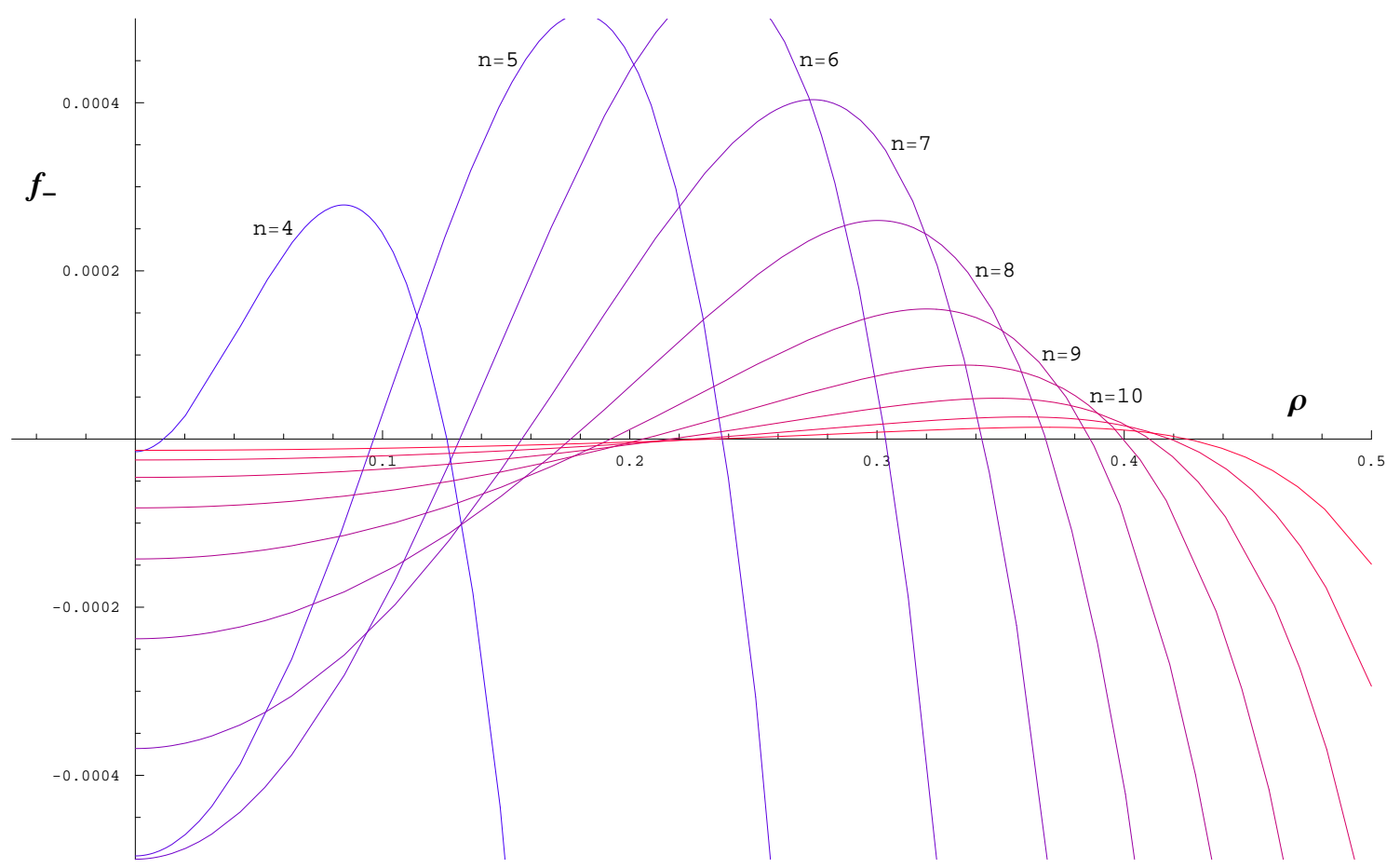

Figure 3.7: Graphs for $f_{-}(\rho)$ for various cases of $n$ and $r=\frac{1}{2}$. Note that at $\rho=0$ all functions are negative and that for each $n$ there are values of $\rho$ between 0 and $\frac{1}{2}$ such that $f_{-}$is positive. The mass is $m_{\star}\left(r=\frac{1}{2}\right) / 2$ and $\rho$ is given in terms of the Einstein radius.

\subsection{Properties of $m_{\star}$ as an Upper Bound}

In this section we study the properties of the upper bound found and described in (3.9) for the central mass in a maximal lensing configuration.

First of all, note that

$$
\frac{\partial m_{\star}}{\partial r}=\frac{2\left((n+5) r^{\frac{n+11}{n-1}}\left(1+r^{6}\right)^{2}-3 r^{5}(n-1)\right)}{(n-1)\left(1+r^{6}\right)^{2}}
$$

By mathematical induction in $n$, it is possible to prove that $\frac{\partial m_{\star}}{\partial r}>0$, as long as $r<1$ and $n \geq 4$ (see Section A.2). As the configurations under study require $n \geq 4$ and $r<r_{c r}$, given that $r_{c r}<1$ (see equation (3.2) and Section A.1), it follows that $\frac{\partial m_{\star}}{\partial r}$ is positive. This in turn implies that $m_{\star}$ increases with the radius of the polygon $r$, so that the farther from the central mass the polygon of masses is located, the larger the mass perturbation that can be placed at the center of the polygon while keeping the maximum 


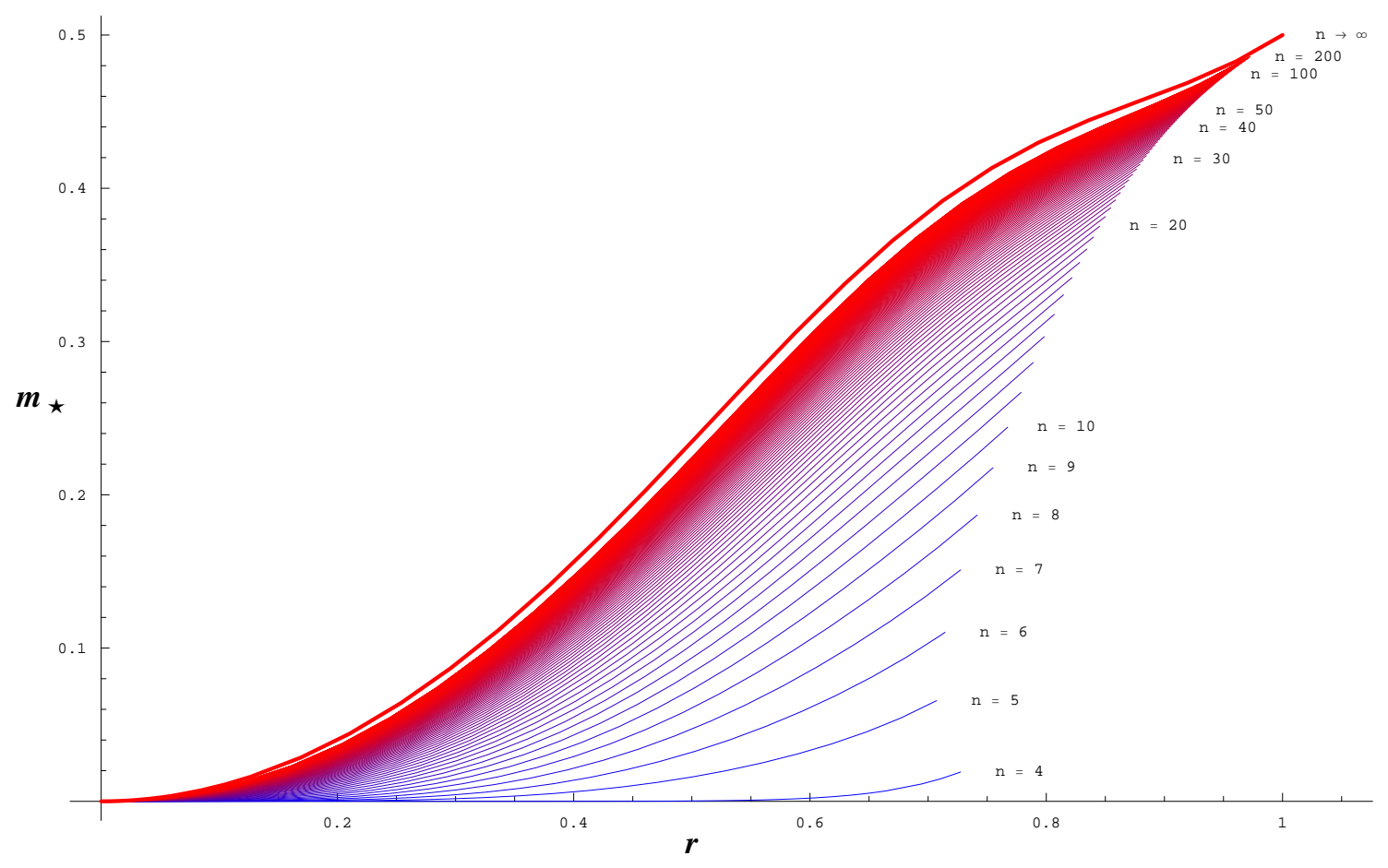

Figure 3.8: The critical mass $m_{\star}$ as a function of the number of lenses $n$ and the radius $r$ of the regular polygon. Mass is given in units of the total mass in the polygon and $r$, given in units of the Einstein radius, is less than the critical radius $r_{c r}$ for each value of $n$.

number of images. This dependence of $m_{\star}$ on the radius $r$ of the polygon is illustrated in Figure 3.8 for various values of $n$.

It is also of interest to study the behaviour of $m_{\star}$ as a function of $n$. To this end, note that

$$
\frac{\partial m_{\star}}{\partial n}=-\frac{12 r^{\frac{2(n+5)}{n-1}}}{(n-1)^{2}} \ln r
$$

Given that $r<1$ it follows from equation (3.11) that $\frac{\partial m_{\star}}{\partial n}>0$, and thus $m_{\star}$ increases with the number of lenses $n$. This in turn implies that as the number of lenses in the polygon increases, the central mass perturbation can be larger and therefore the system is more stable to perturbations in the central mass. The dependence of $m_{\star}$ on the number of lenses $n$ is illustrated in Figure 3.8.

The sharpness of $m_{\star}$ as an upper bound was studied through simulations (see Section B.1). Our study on the behaviour and stability of the maximal lensing configuration of a polygon of lenses perturbed by a small central mass initially focused on the case of 
$r=\frac{1}{2}$. A semi-analytic approach in this particular case led to the values of $\rho_{0}$ and $m_{\star}$ described in equations (3.8) and (3.9). As an example, consider a configuration of 6 point-masses of mass $m=\frac{1}{6}$ each, acting as lenses at the vertices of a regular hexagon of radius $r=\frac{1}{2}<r_{c r}$. With the source located at $z_{s}=0$ and the small mass perturbation $m$ at the center of the configuration, a maximal lensing configuration is obtained if $m<m_{\star} \cong 0.047$. This maximal lensing configuration can be found in Figure 3.9 for $m=\frac{3}{100}<m_{\star}$, producing a total of $N_{\max }=30$ images. In contrast, Figure 3.10 illustrates the same configuration for $m=\frac{6}{100}>m_{\star}$, producing only $N=18$ images.

The simulations performed for $r=\frac{1}{2}$ and various values of $n$ allowed us to find limits on the central mass. This in turn leads to a curve to which it was possible to fit an analytic expression for the upper bound. These results from the simulations were in turn confirmed using the expression found for $f_{-}(\rho)$ in equation (3.7) to guarantee the maximum number of images. For $r=\frac{1}{2}$, Figure 3.11 is the plot of $m_{\star}$ along with the points found through simulations that represent the maximum mass that allows the system to produce the maximum number of images. The good fit is confirmed by a $\chi^{2}$ test.

The same type of simulations performed for the values of $r=\frac{1}{10}, r=\frac{1}{4}$ and $r=\frac{7}{10}$ confirm that $m_{\star}$ is an upper bound on the central mass for maximal lensing. However, this bound is not sharp so that larger masses can lead to maximal lensing. In other words, $m<m_{\star}$ is a sufficient condition for maximal lensing but it is not a necessary condition. Illustrated in Figures 3.12, 3.13 and 3.14 are the plots for $m_{\star}$ along with the maximum masses found through simulations for $r=\frac{1}{10}, r=\frac{1}{4}$ and $r=\frac{7}{10}$ respectively, where the poor fits are further evidence that $m_{\star}$ is not a sharp bound. 


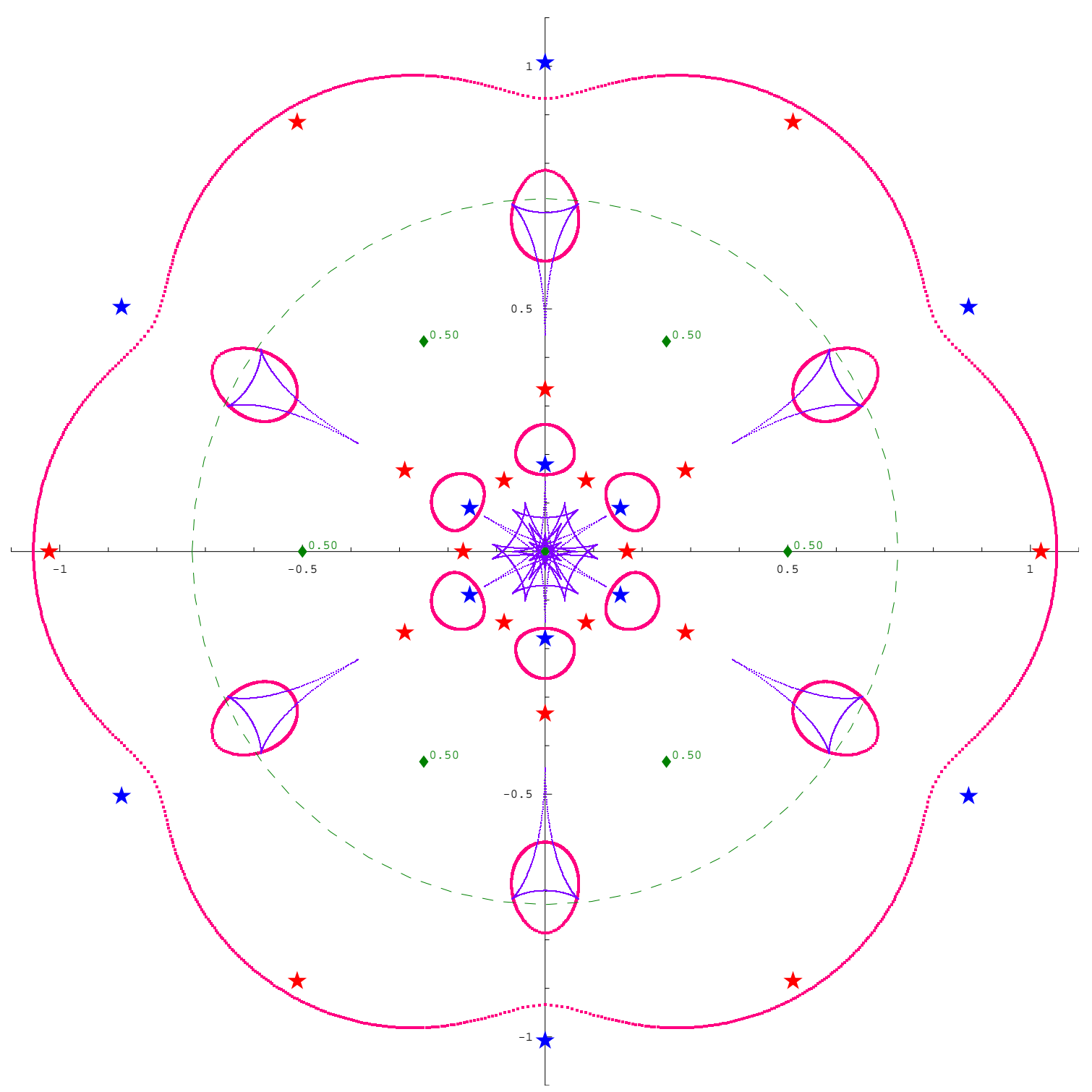

Figure 3.9: A configuration of point-mass lenses in a regular hexagon perturbed by a small central mass of $m=\frac{3}{100}<m_{\star}$. The dashed line represents the circle of radius $r_{c r} \cong 0.73$. Lenses at the vertices of the pentagon, indicated with diamonds, are located at $r=0.50<r_{c r}$. This configuration produces the maximum number of images $N=30$ for a set of $n=7$ point lenses. Images are represented by five-pointed stars, while thick smooth lines represent critical curves and thin cuspy lines represent the caustic curves. 


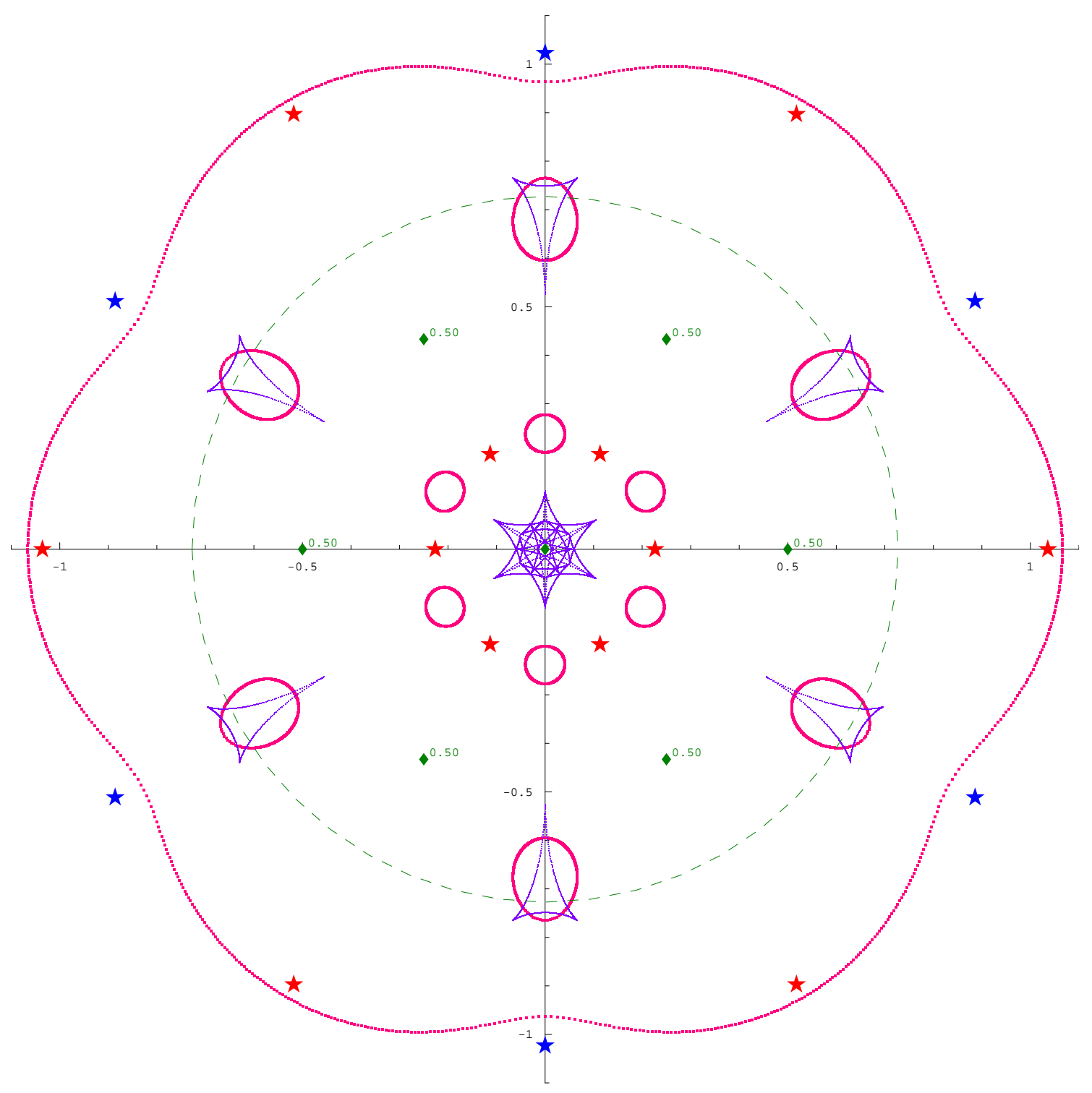

Figure 3.10: A configuration of point-mass lenses in a regular hexagon perturbed by a small central mass of $m=\frac{6}{100}>m_{\star}$. The dashed line represents the circle of radius $r_{c r} \cong 0.73$. Lenses at the vertices of the pentagon, indicated with diamonds, are located at $r=0.50<r_{c r}$. This configuration produces only $N=18$ images, less than the maximum possible for a set of $n=7$ point lenses. Images are represented by five-pointed stars, while thick smooth lines represent critical curves and thin cuspy lines represent the caustic curves. 


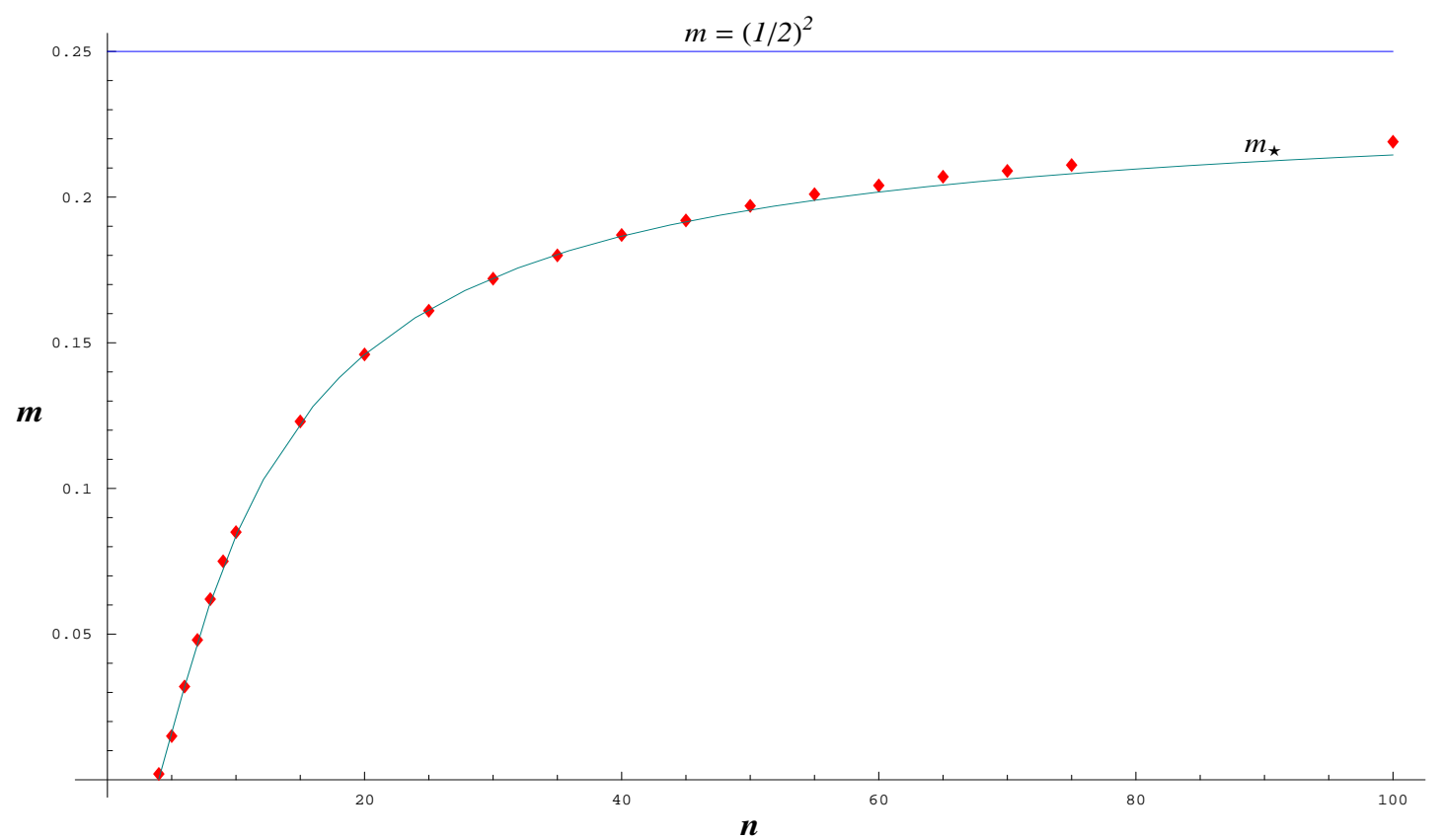

Figure 3.11: Maximum mass for maximal lensing for $r=\frac{1}{2}$ as a function of the number of lenses $n$. Points represent the maximum mass found through simulations and the smooth curve represents the analytic bound $m_{\star}$. Point-size is larger than the error bars and $\chi^{2}=0.86$ per degree of freedom. The mass, given in units of the total mass in the polygon, asymptotically approaches $m=\left(\frac{1}{2}\right)^{2}$ with the increase in the number of lenses.

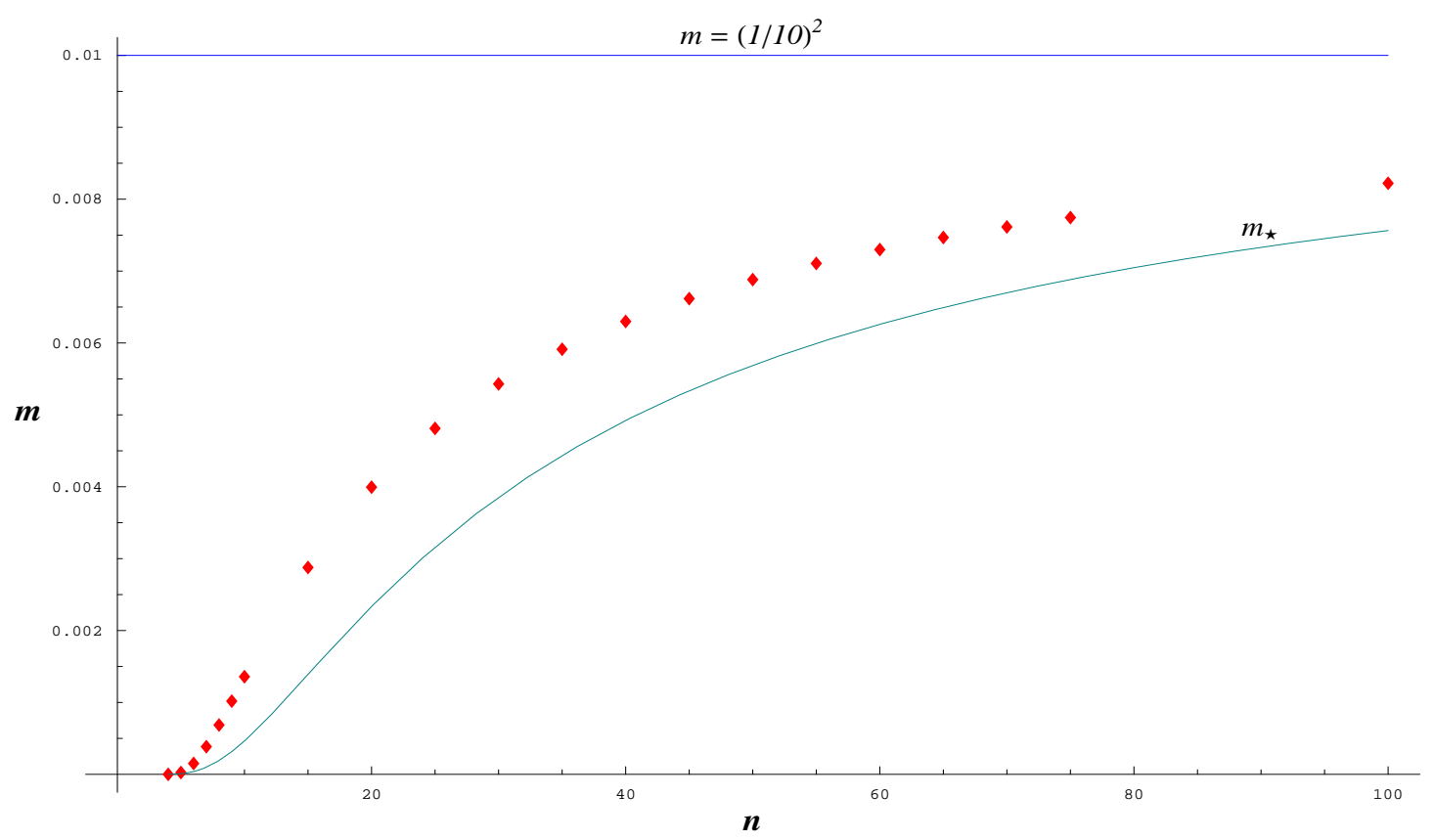

Figure 3.12: Maximum mass for maximal lensing for $r=\frac{1}{10}$ as a function of the number of lenses $n$. Points represent the maximum mass found through simulations and the smooth curve represents the analytic bound $m_{\star}$. The mass, given in units of the total mass in the polygon, asymptotically approaches $m=\left(\frac{1}{10}\right)^{2}$ with the increase in the number of lenses. 


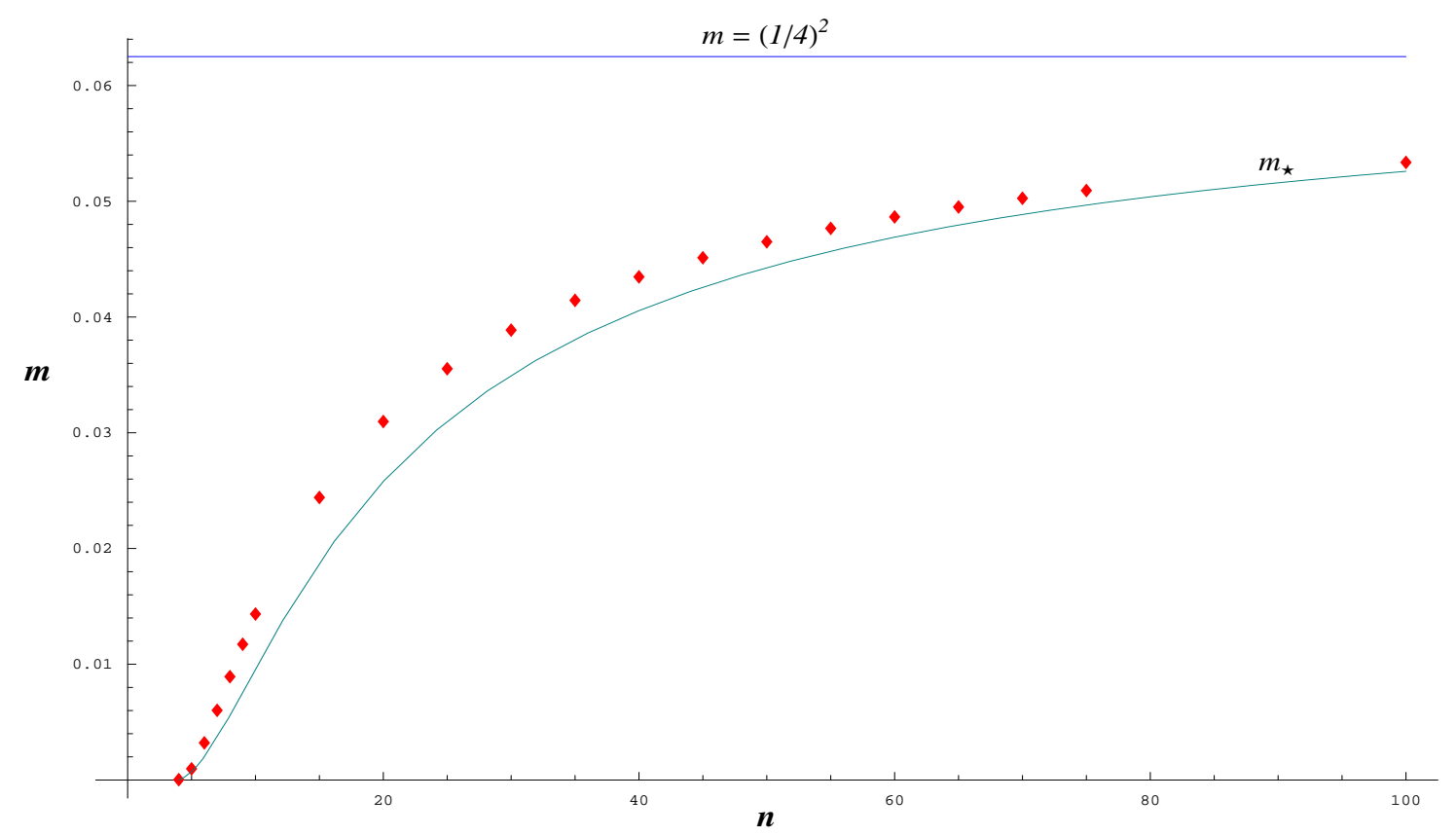

Figure 3.13: Maximum mass for maximal lensing for $r=\frac{1}{4}$ as a function of the number of lenses $n$. Points represent the maximum mass found through simulations and the smooth curve represents the analytic bound $m_{\star}$. The mass, given in units of the total mass in the polygon, asymptotically approaches $m=\left(\frac{1}{4}\right)^{2}$ with the increase in the number of lenses.

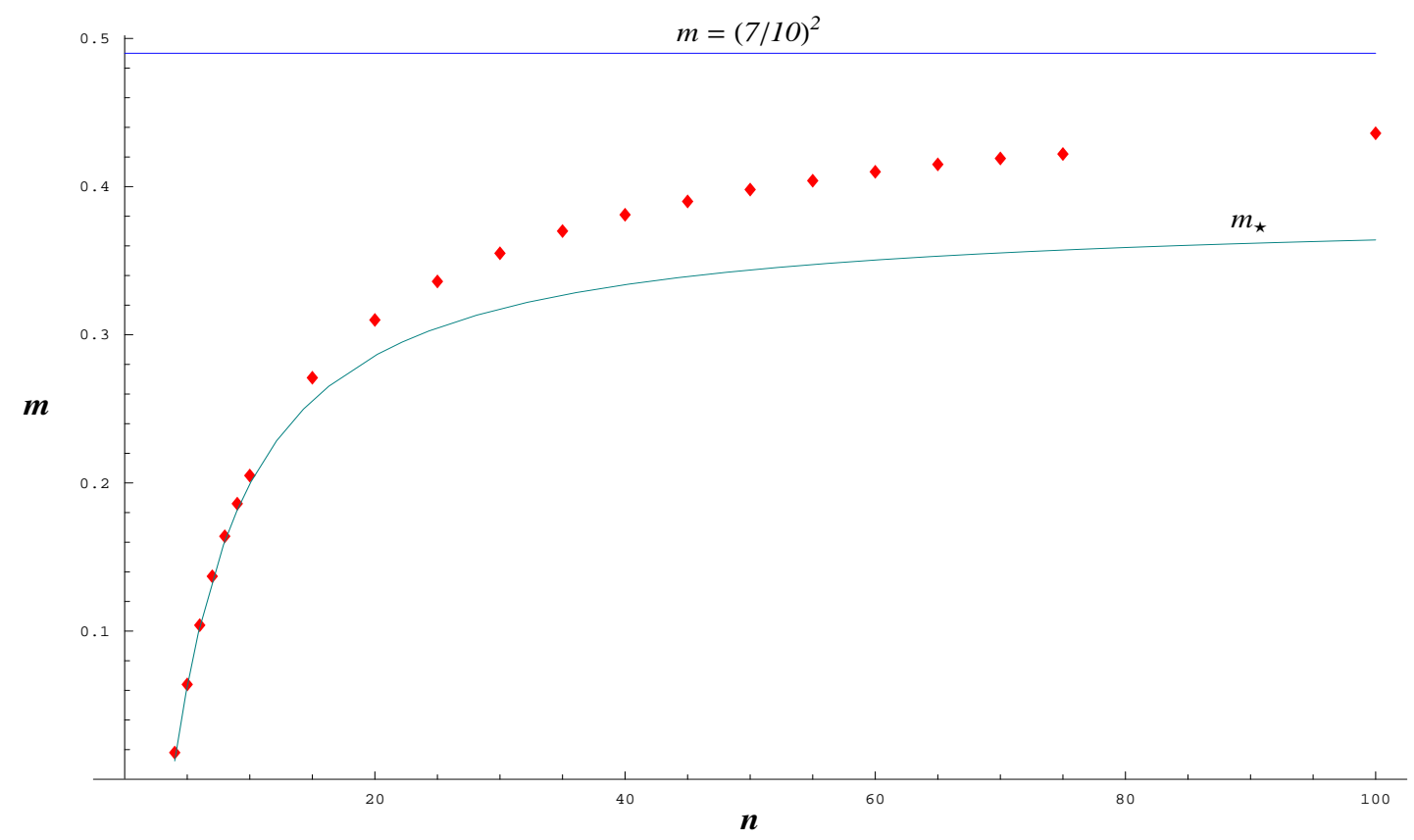

Figure 3.14: Maximum mass for maximal lensing for $r=\frac{7}{10}$ as a function of the number of lenses $n$. Points represent the maximum mass found through simulations and the smooth curve represents the analytic bound $m_{\star}$. The mass, given in units of the total mass in the polygon, asymptotically approaches $m=\left(\frac{7}{10}\right)^{2}$ with the increase in the number of lenses. 


\subsection{Summary}

In this chapter we studied a gravitational lensing scenario for $n \geq 4$ point-mass deflectors. Initially, $n-1$ deflectors of equal mass are arranged at the vertices of a regular polygon of $n-1$ sides and of radius $r<\frac{1}{\sqrt{2}}$. The lens equation was then normalized so that all masses were expressed in terms of the total mass of the lenses in the polygon and distances in the lens plane were measured in terms of the Einstein radius of the same total mass.

If a perturbation of mass $m$ is located at the center of the polygon, we proved that whenever $m<m_{\star}$ for

$$
m_{\star}=r^{2\left(\frac{n+5}{n-1}\right)}-\frac{r^{6}}{1+r^{6}},
$$

the lensing system produces the maximum of $5 n-5$ images of a distant source. 


\section{Chapter 4}

\section{Lensing and Distributed Lenses}

The results outlined in Chapter 2 and extended further in Chapter 3 settle the question of maximal lensing for point-mass lenses in a single plane or in a distribution that can be considered thin. However, real lenses in many cases of astrophysical interest rarely exhibit such a singular mass distribution. With the exception of a Schwarzschild black hole and other singularities, the description of deflecting masses by point-mass lenses is an approximation.

The Odd Number Theorem discussed in Section 2.2 states that for realistic mass distributions, the number of images is odd. This is clearly not the case in the maximal lensing result found for point-mass lenses as $5 n-5$ is even for all odd values of $n$. Furthermore, even though almost all observed cases of multiple imaging exhibit an even number of images, it is well known that for most realistic mass profiles of the lensing distribution, the missing image is highly demagnified and located near the center of the deflector thus making it hard to detect (see Narasimha et al., 1986).

The negative results for the observation of central images have been used to probe and set constraints on the mass profiles of galaxies and the mass of super massive black holes at the center of lensing galaxies (see Rusin et al., 2005, Boyce et al., 2005, Boyce et al., 2006 and Dobler et al., 2007). Furthermore, the first observed central image in 
radio wavelengths (see Winn et al., 2004) and the first observed central image at optical wavelengths (see Inada et al., 2005) have proved useful in characterizing the density of the lensing galaxy as well as the mass of its central black hole.

In this section we extend the maximal lensing results from point-mass lenses to realistic distributed lenses by proving that in a point-mass lens configuration, the effect of replacing one point mass with a distributed lens amounts to the addition of one extra image located inside the radius of the distributed lens.

\subsection{Preliminaries}

To extend the maximal lensing results from point-mass configurations to more realistic distributed masses some previous results will be used.

As discussed in Chapter 2, the lens equation for a set of $n$ point masses acting as deflectors takes the form (see equation 2.13)

$$
z_{s}=z-\sum_{k=1}^{n} \frac{m_{k}}{\bar{z}-\bar{z}_{k}}
$$

where $m_{k}$ is the mass of the $k^{\text {th }}$ point lens located at $z_{k}$ on the lens plane and $z_{s}$ is the position of the source projected on the lens plane. This equation is to be solved for $z$ to find the positions of the images formed by the configuration.

Recall also that in Chapter 3 we proved that a configuration of $n$ point-mass lenses produces a maximum of $5 n-5$ images. Furthermore, an explicit maximal lensing configuration consists of $n-1$ identical point masses located at the vertices of a regular polygon of $n-1$ sides, plus a small mass located at the center of the polygon.

The term distributed lens will be used from this point onwards to represent a deflecting mass of finite non-zero radius. Without loss of generality, we require the mass distribution to have spherical symmetry so that when projected on the lens plane, the resulting surface mass density has cylindrical symmetry. Furthermore, the mass $\tilde{m}(h)$ inside a cylinder of 
radius $h$ can be written in terms of the projected mass density $\sigma(h)$ as

$$
\tilde{m}(h)=2 \pi \int_{0}^{h} \sigma\left(h^{\prime}\right) h^{\prime} d h^{\prime} .
$$

For this particular case of spherical symmetry in the mass distribution, the bending angle is given in equation (1.47) as

$$
\alpha=\frac{4 G \tilde{m}(h)}{c^{2} h}
$$

Thus, unlike the point-mass lens, a distributed lens leads to a cylindrical mass $\tilde{m}(h)$ and bending angle $\alpha$ that remain finite as long as the surface density $\sigma(h)$ does not diverge faster than $h^{-1}$ as $h \rightarrow 0$ at the center of the mass distribution (see equations 4.2 and 4.3)

\subsection{From Point Masses to Distributed Lenses}

The goal of this section is to prove that in a configuration of point-mass lenses, when one lens is replaced with a distributed lens of a radius small enough so that it does not overlap with other lenses or existing images, a new image forms inside the distributed lens. Thus, the total number of images will increase by one as the images outside the distributed lens are not affected. This method is robust in that no symmetries are assumed in the initial configuration of point-mass lenses; any arrangement of point masses may be used and any of those point masses can be replaced with a distributed lens.

If $n$ point-mass lenses are systematically replaced by distributed lenses, avoiding overlap with existing images and lenses, the number of images will increase by $n$. Thus, in a point-mass configuration where the maximum number of images is attained, the maximum will increase by $n$ when all the point masses are replaced by distributed lenses.

In order to prove the result a mass distribution that will be used to replace one of 
the point-mass lenses must be specified. The most straightforward lens distribution to choose in this situation is one of constant projected mass density given by

$$
\sigma(h)=\left\{\begin{array}{cc}
\sigma_{0} & \text { for } h \leq H \\
0 & \text { for } h>H
\end{array}\right.
$$

where $H$ is the radius of the lens. As previously mentioned, $H$ is constrained to be small enough to avoid overlap with other lenses and existing images. This choice of mass distribution is not only motivated by ease of calculation, but it is also generic in that for all finite core mass densities that cause finite bending, this bending angle goes to zero as the center is approached, as it happens in the case of constant projected mass density.

With $\sigma(h)$ given in equation (4.4), the cylindrical mass in equation (4.2) can be calculated as

$$
\tilde{m}(h)=\sigma_{0} \pi h^{2}=\frac{M}{H^{2}} h^{2} \quad \text { for } h \leq H
$$

where $M$ is the total mass of the lens of radius $H$.

Replacing the $n^{\text {th }}$ point lens at position $z_{n}$ in the configuration described by (4.1) with a distributed lens of cylindrical mass $\tilde{m}(h)$, the lens equation becomes

$$
z_{s}=z-\sum_{k=1}^{n-1} \frac{m_{k}}{\bar{z}-\overline{z_{k}}}-\frac{\tilde{m}\left(\left|z-z_{n}\right|\right)}{\bar{z}-\bar{z}_{n}}
$$

If the total mass $M$ of the distributed lens is equal to the point mass $m_{n}$ being replaced, the number and positions of images outside the radius $H$ remain unchanged. Therefore, from this point on we will be only interested in images and solutions to the lens equation that lie inside the radius of the distributed lens, so that $h=\left|z-z_{n}\right|$ is less than $H$. 
With the cylindrical mass calculated in (4.5), the lens equation (4.6) becomes

$$
z_{s}=z-\sum_{k=1}^{n-1} \frac{m_{k}}{\bar{z}-\overline{z_{k}}}-\frac{M}{H^{2}} \frac{\left|z-z_{n}\right|^{2}}{\bar{z}-\bar{z}_{n}}
$$

and then simplified to

$$
z_{s}=z-\sum_{k=1}^{n-1} \frac{m_{k}}{\bar{z}-\overline{z_{k}}}-\frac{M}{H^{2}}\left(z-z_{n}\right) .
$$

Since we are looking for new images formed, we need to find solutions for this lens equation such that $\left|z-z_{n}\right| \leq H$. Given that the inversion of (4.7) cannot be carried out analytically it is then necessary to find an approximate method. The most straightforward way to carry out this procedure is to position the source at $z_{s}=0$ and Taylor-expand the terms in the sum in equation (4.7) about $z=z_{n}$.

Note that it is not possible to expand the entire lensing map since equation (4.7) is not analytic due to its irreducible dependence on $\bar{z}$. However, $1 /\left(z-z_{k}\right)$ is analytic at $z_{n}$ since $z_{k} \neq z_{n}$, and using Weierstrass theorem on the approximation by polynomials (see for instance Butkov, 1968 or any textbook on Complex Analysis), the Taylor expansion of $1 /\left(z-z_{k}\right)$ is uniformly convergent in a neighborhood of $z_{n}$. Therefore, the terms of this expansion for $1 /\left(z-z_{k}\right)$ can be reorganized and grouped into two separate series: one for the real part and one for the imaginary part. Taking the complex conjugate of each one of these series results in expansions for each term of the form $1 /\left(\bar{z}-\bar{z}_{k}\right)$ that appears in the sum of equation (4.7).

As a result, uniform convergence allows for the second term in equation (4.7) to be written as

$$
\sum_{k=1}^{n-1} \frac{1}{\bar{z}-\bar{z}_{k}}=U(x, y)+i V(x, y),
$$

where $U(x, y)$ and $V(x, y)$ are respectively the real and imaginary parts of the function and $z=x+i y$. Due to the analyticity of equation (4.8), the series for $U(x, y)$ and 
$V(x, y)$ must be convergent. Given that

$$
\sum_{k=1}^{n-1} \frac{1}{\bar{z}-\bar{z}_{k}}=\sum_{k=1}^{n-1} \frac{z-z_{k}}{\left|z-z_{k}\right|^{2}}
$$

the values for $U(x, y)$ and $V(x, y)$ in $(4.8)$ can be expressed as

$$
U(x, y)=\sum_{k=1}^{n-1} \frac{x-x_{k}}{\left(x-x_{k}\right)^{2}+\left(y-y_{k}\right)^{2}}
$$

and

$$
V(x, y)=\sum_{k=1}^{n-1} \frac{y-y_{k}}{\left(x-x_{k}\right)^{2}+\left(y-y_{k}\right)^{2}},
$$

where $z_{k}=x_{k}+i y_{k}$ for the position of the point-mass lenses.

In order to perform the approximation and make explicit the first terms in the Taylor expansion, the first order derivatives needed are

$$
\begin{aligned}
\frac{\partial U}{\partial x} & =\sum_{k=1}^{n-1} \frac{\left(y-y_{k}\right)^{2}-\left(x-x_{k}\right)^{2}}{\left(\left(x-x_{k}\right)^{2}+\left(y-y_{k}\right)^{2}\right)^{2}} & \frac{\partial U}{\partial y} & =\sum_{k=1}^{n-1} \frac{-2\left(x-x_{k}\right)\left(y-y_{k}\right)}{\left(\left(x-x_{k}\right)^{2}+\left(y-y_{k}\right)^{2}\right)^{2}}, \\
\frac{\partial V}{\partial x} & =\sum_{k=1}^{n-1} \frac{-2\left(x-x_{k}\right)\left(y-y_{k}\right)}{\left(\left(x-x_{k}\right)^{2}+\left(y-y_{k}\right)^{2}\right)^{2}} & \frac{\partial V}{\partial y} & =\sum_{k=1}^{n-1} \frac{\left(x-x_{k}\right)^{2}-\left(y-y_{k}\right)^{2}}{\left(\left(x-x_{k}\right)^{2}+\left(y-y_{k}\right)^{2}\right)^{2}} .
\end{aligned}
$$

The first order two dimensional Taylor expansions for $U(x, y)$ and $V(x, y)$ around the center of the distributed lens $(x, y)=\left(x_{n}, y_{n}\right)$, are thus given by

$$
U(x, y) \approx \sum_{k=1}^{n-1} \frac{X_{k}}{h_{k}^{2}}+\sum_{k=1}^{n-1} \frac{Y_{k}^{2}-X_{k}^{2}}{h_{k}^{4}}\left(x-x_{n}\right)-\sum_{k=1}^{n-1} \frac{2 X_{k} Y_{k}}{h_{k}^{4}}\left(y-y_{n}\right)
$$

and

$$
V(x, y) \approx \sum_{k=1}^{n-1} \frac{Y_{k}}{h_{k}^{2}}+\sum_{k=1}^{n-1} \frac{X_{k}^{2}-Y_{k}^{2}}{h_{k}^{4}}\left(y-y_{n}\right)-\sum_{k=1}^{n-1} \frac{2 X_{k} Y_{k}}{h_{k}^{4}}\left(x-x_{n}\right),
$$

where for readability we have defined $X_{k} \equiv x_{n}-x_{k}, Y_{k} \equiv y_{n}-y_{k}$ and $h_{k}^{2} \equiv X_{k}^{2}-Y_{k}^{2}$.

Substituting the values of the approximations (4.9) and (4.10) into equation (4.7) and locating the source at $z_{s}=0$, the real and imaginary parts of the lens equation are 
respectively

$$
0=x-\sum_{k=1}^{n-1} \frac{m_{k} X_{k}}{h_{k}^{2}}-\left(\frac{M}{H^{2}}+\sum_{k=1}^{n-1} \frac{m_{k}\left(Y_{k}^{2}-X_{k}^{2}\right)}{h_{k}^{4}}\right)\left(x-x_{n}\right)+\sum_{k=1}^{n-1} \frac{2 m_{k} X_{k} Y_{k}}{h_{k}^{4}}\left(y-y_{n}\right)
$$

and

$$
0=y-\sum_{k=1}^{n-1} \frac{m_{k} Y_{k}}{h_{k}^{2}}-\left(\frac{M}{H^{2}}+\sum_{k=1}^{n-1} \frac{m_{k}\left(X_{k}^{2}-Y_{k}^{2}\right)}{h_{k}^{4}}\right)\left(y-y_{n}\right)+\sum_{k=1}^{n-1} \frac{2 m_{k} X_{k} Y_{k}}{h_{k}^{4}}\left(x-x_{n}\right)
$$

Setting $X \equiv x-x_{n}$ and $Y \equiv y-y_{n}$, and defining the constants

$$
\begin{gathered}
A=\sum_{k=1}^{n-1} \frac{m_{k} X_{k}}{h_{k}^{2}}-x_{n}, \quad B=\sum_{k=1}^{n-1} \frac{m_{k} Y_{k}}{h_{k}^{2}}-y_{n} \\
C=\frac{M}{H^{2}}+\sum_{k=1}^{n-1} \frac{m_{k}\left(Y_{k}^{2}-X_{k}^{2}\right)}{h_{k}^{4}}, \quad D=\frac{M}{H^{2}}+\sum_{k=1}^{n-1} \frac{m_{k}\left(X_{k}^{2}-Y_{k}^{2}\right)}{h_{k}^{4}} \\
E=\sum_{k=1}^{n-1} \frac{2 m_{k} X_{k} Y_{k}}{h_{k}^{4}},
\end{gathered}
$$

equations (4.11) and (4.12) can be written more compactly as

$$
0=X-A-C X+E Y
$$

and

$$
0=Y-B-D Y+E X
$$

Simultaneously solving equations (4.13) and (4.14) we obtain

$$
X=\frac{A(1-D)-B E}{1-D-C+C D-E^{2}}
$$

and

$$
Y=\frac{B(1-C)-A E}{1-D-C+C D-E^{2}}
$$

Since $X=x-x_{n}$ and $Y=y-y_{n}$, the position of this image is therefore given by 
$z=x+i y$, where

$$
x=\frac{A(1-D)-B E}{1-D-C+C D-E^{2}}+x_{n}
$$

and

$$
y=\frac{B(1-C)-A E}{1-D-C+C D-E^{2}}+y_{n} .
$$

Given that images outside the radius $H$ of the distributed lens have positions $(x, y)$ that do not depend on the size of the distributed lens, these exterior images must obey $\frac{\partial x}{\partial H}=0$ and $\frac{\partial y}{\partial H}=0$. Conversely, any image $(x, y)$ for which $\frac{\partial x}{\partial H} \neq 0$ or $\frac{\partial y}{\partial H} \neq 0$, must lie inside the distributed lens. Direct derivation from equations (4.15) and (4.16) results in

$$
\frac{\partial x}{\partial H}=\frac{-2 H M\left(2 B E H^{2}\left(M-H^{2}\right)+A\left(M^{2}-2 H^{2} M(F+1)+H^{4}\left(E^{2}+(F+1)^{2}\right)\right)\right)}{\left(2 H^{2} M-M^{2}+H^{4}\left(E^{2}+F^{2}-1\right)\right)^{2}} \neq 0
$$

and

$$
\frac{\partial y}{\partial H}=\frac{-2 H M\left(2 A E H^{2}\left(M-H^{2}\right)+B\left(M^{2}+2 H^{2} M(F-1)+H^{4}\left(E^{2}+(F-1)^{2}\right)\right)\right)}{\left(2 H^{2} M-M^{2}+H^{4}\left(E^{2}+F^{2}-1\right)\right)^{2}} \neq 0,
$$

where

$$
F \equiv \sum_{k=1}^{n-1} \frac{m_{k}\left(Y_{k}^{2}-X_{k}^{2}\right)}{h_{k}^{4}} .
$$

The non-zero derivatives found in equations (4.17) and (4.18) guarantee that the new solution found lies inside the radius of the distributed lens.

Images existing prior to the conversion of one of the point-mass lenses into a distributed mass are not affected by the extended mass distribution. Thus, the net effect of converting one of the point lenses to a distributed lens is the addition of one more image located within the radius of the extended mass. Furthermore, this procedure can be carried out replacing all point-mass lenses with distributed masses. As long as the radius of each distributed lens is small enough to prevent it from overlapping with existing images and neighboring lenses, each lens thus replaced adds one more image, while leaving the rest of the images unaffected. 


\subsection{Summary}

We have shown that when a configuration of point-mass lenses is modified by replacing one of the point masses with a distributed mass deflector, a new image forms within the radius of the distributed lens. Thus, the total number of images increases by one, while leaving all other images unaffected. The result is generic and applies to any mass distribution with a projected density that remains finite at the center, ensuring that the leading term is constant. The density profile is then only constrained to have a core of finite size and should diverge strictly slower than $h^{-1}$ as $h$ approaches the center of the distribution.

By systematically replacing all the lenses in a point-mass lens configuration with distributed masses and constraining the lenses from overlapping with each other or with existing images, the number of images in a configuration of $n$ lenses will increase by $n$. This implies that the maximum number of images for a system of $n$ distributed and nonoverlapping lenses is $(5 n-5)+n=6(n-1)+1$. Furthermore, this result is consistent with the Odd Number Theorem discussed in Section 2.2. 


\section{Chapter 5}

\section{Stability and Applications}

In this chapter we study the stability properties of the maximal lensing configurations found for point-mass lenses, as well as the possible applications of these special gravitational lensing systems. The codes for the simulations used in this chapter can be found in Appendix B.

In Section 5.1 we present results on the stability properties of the point-mass lens configurations that exhibit maximal lensing, as described in Chapter 3.

In Section 5.2 we illustrate an application of astrophysical interest derived from the upper bound mass $m_{\star}$ found in equation (3.9).

In Section 5.3 we present examples of asymmetric maximal lensing configurations found through simulations and in Section 5.4 we study and compare the stability of these asymmetric configurations against the stability properties of the corresponding symmetric maximal lensing configurations with a central mass perturbation.

\subsection{Perturbations on Symmetric Configurations}

Recall that a set of $n$ point-mass lenses, when arranged so that $n-1$ of equal mass are located at the vertices of a regular polygon of $n-1$ sides, will produce the maximum number of images of a source with position that projects at the center of the polygon on 
the lens plane, as long as the remaining point-mass is located at the center of the polygon and has a mass bounded by $m_{\star}$ according to equation (3.9). The degree of symmetry in the configuration raises the question of how stable is this maximal lensing scenario actually. We study the stability through simulations that describe several perturbations on the maximal lensing configuration. The specific code for these simulations can be found in Section B.1.

The values of $n$ used in the perturbations lie in the range $n=4, \ldots, 14$ as larger values exceed the computational resources available. Given that for each value of $n$ there is a different and corresponding value of $r_{c r}$ that describes the maximum radius of the polygon (see equation 3.1), we set $r=\frac{r_{c r}}{2}$ as the radius of the polygon for the configuration to be perturbed. Furthermore, recall that each lens at a vertex of the polygon has a mass of $m=\frac{1}{n-1}$. According to the main result proved in Chapter 3, a sufficient condition for the system to produce the maximum number of images is that, for the corresponding values of $n$ and $r$, the central mass should be smaller than $m_{\star}$. Using the results from the simulations described in Section B.1 and from the properties of $m_{\star}$ studied in Section 3.2 , we set $m=\frac{m_{\max }}{2}$ for the central mass in the configuration to be perturbed, where $m_{\max }$ is the maximum central mass for the polygon of radius $r=\frac{r_{c r}}{2}$ in a maximal lensing configuration.

To test the stability of the configuration under changes in the position of one of the point lenses located at a vertex of the polygon, one of these lenses had its radial and tangential coordinates perturbed away from the original symmetric configuration. Through the simulations we find the range of values in the radial and tangential coordinates that keep the number of images maximum. This in turn is used to calculate the intervals $\Delta r$ and $\Delta \theta$ available for maximal lensing around the initial symmetric configuration. To compare the allowed values with the available phase space we define an ellipse with semi-major axis $a=\frac{\Delta r}{2}$ and semi-minor axis $b=\frac{\Delta \theta}{2} \frac{r_{c r}}{2}$, and consider its area $A_{\text {ellipse }}=\pi \frac{\Delta r \Delta \theta}{8} r_{c r}$ as representative of the region of stability for maximal lensing. Since 


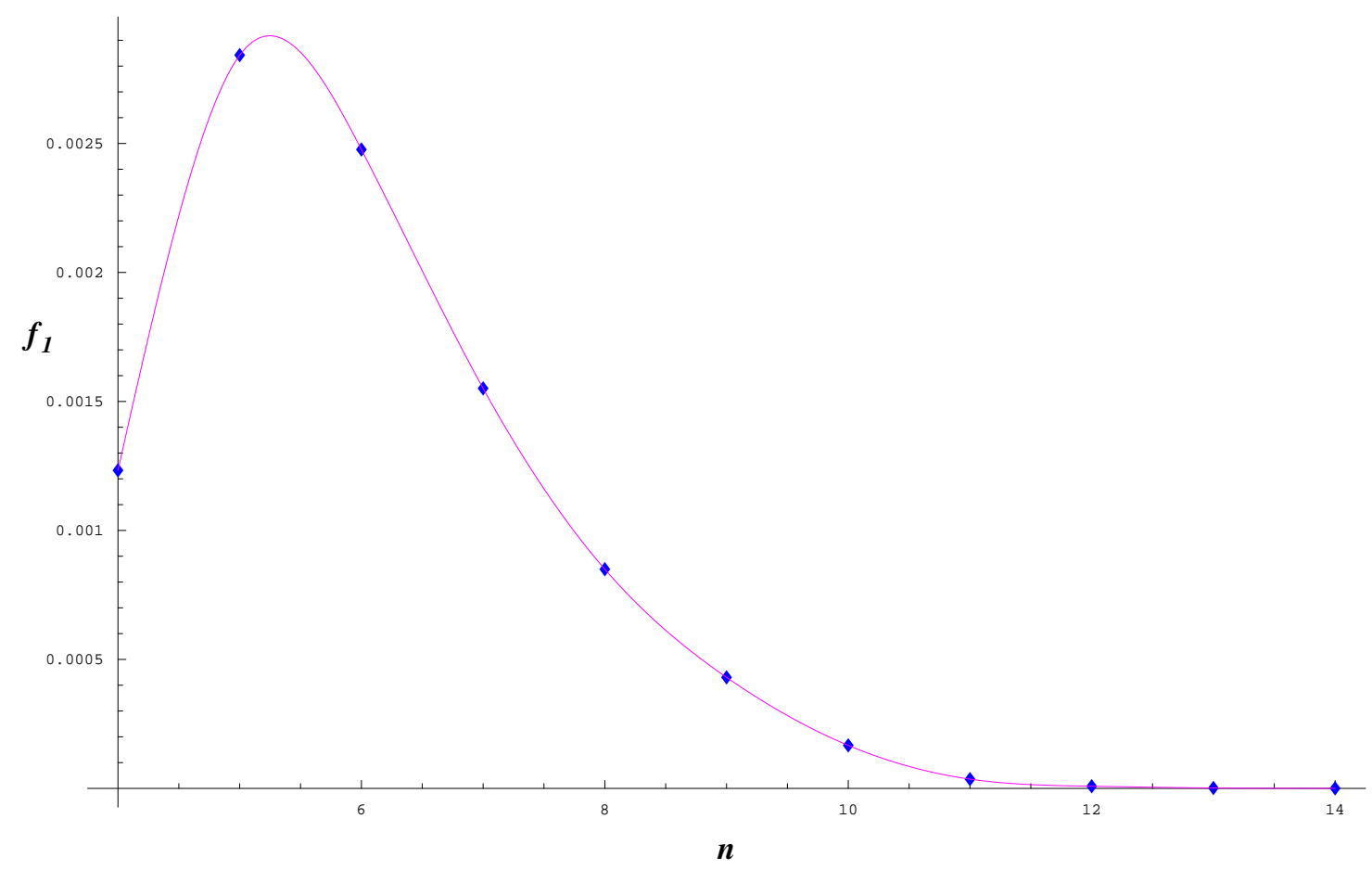

Figure 5.1: Fraction $f_{1}$ of the critical area of the lens under which maximal lensing is present, when the position of a lens on the polygon is perturbed. Instability increases with the number of lenses $n$. Fractions are in percentage units of the area of the critical disk.

the total area available for the lensing configuration is set by the disk of critical radius $r_{c r}$, a measure of the stability of the configuration under a perturbation in the position of one of the lenses at a vertex of the polygon is the fraction $f_{1}$ given by

$$
f_{1} \equiv \frac{A_{\text {ellipse }}}{A_{r_{c r}}}=\frac{\Delta r \Delta \theta}{8 r_{c r}} .
$$

From the simulations, the maximum value for $f_{1}$ is $0.003 \%$ at $n=5$, with an exponential decrease with increasing $n$. Thus, the system is highly unstable to perturbations in the positions of lenses at the vertices of the regular polygon. The behaviour of $f_{1}$ as a function of $n$ is illustrated in Figure 5.1.

To test the stability of the configuration under changes in the position of the central mass, the position of this central lens was perturbed away from the initial value of $(0,0)$, both in the horizontal and vertical axes. Through the simulations we find the range of values in the horizontal and vertical coordinates that keep the number of images 
maximum. This in turn is used to calculate the intervals $\Delta x$ and $\Delta y$ available for maximal lensing around the initial symmetric configuration. To compare the allowed values with the available phase space, in a similar way as that used for the perturbation on the position of a lens at a vertex of the polygon, we define an ellipse with semimajor axis $a=\frac{\Delta x}{2}$ and semi-minor axis $b=\frac{\Delta y}{2}$, and consider its area $A_{\text {ellipse }}=\pi \frac{\Delta x \Delta y}{4}$ as representative of the region of stability for maximal lensing. Since the total area available for the lensing configuration is the one set by the disk of critical radius $r_{c r}$, a measure of the stability of the configuration under a perturbation in the position of the central lens is the fraction $f_{2}$ given by

$$
f_{2} \equiv \frac{A_{\text {ellipse }}}{A_{r_{c r}}}=\frac{\Delta x \Delta y}{4 r_{c r}^{2}} .
$$

From the simulations, the maximum value for $f_{2}$ is $0.009 \%$ at $n=5$, with an exponential decrease with increasing $n$. Thus, the system is highly unstable to perturbations in the position of the central mass. The behaviour of $f_{2}$ as a function of $n$ is illustrated in Figure 5.2.

The position of the source was perturbed away from the initial projected value of $(0,0)$, both in the horizontal and vertical axes, in order to test the stability of the configuration under changes in the position of the source. Through the simulations we find the range of values in the horizontal and vertical coordinates that keep the number of images maximum. As done for perturbations on the position of the central mass, the information from the simulations is used to calculate the intervals $\Delta x$ and $\Delta y$ available for maximal lensing around the initial symmetric configuration. To compare the allowed values with the available phase space, an ellipse of area $A_{\text {ellipse }}=\pi \frac{\Delta x \Delta y}{4}$ is defined as representative of the region of stability for maximal lensing. A comparison against the area of the disk of critical radius $r_{c r}$ gives a measure of the stability of the configuration under a perturbation in the position of the source, and can be represented by the fraction $f_{3}$ 


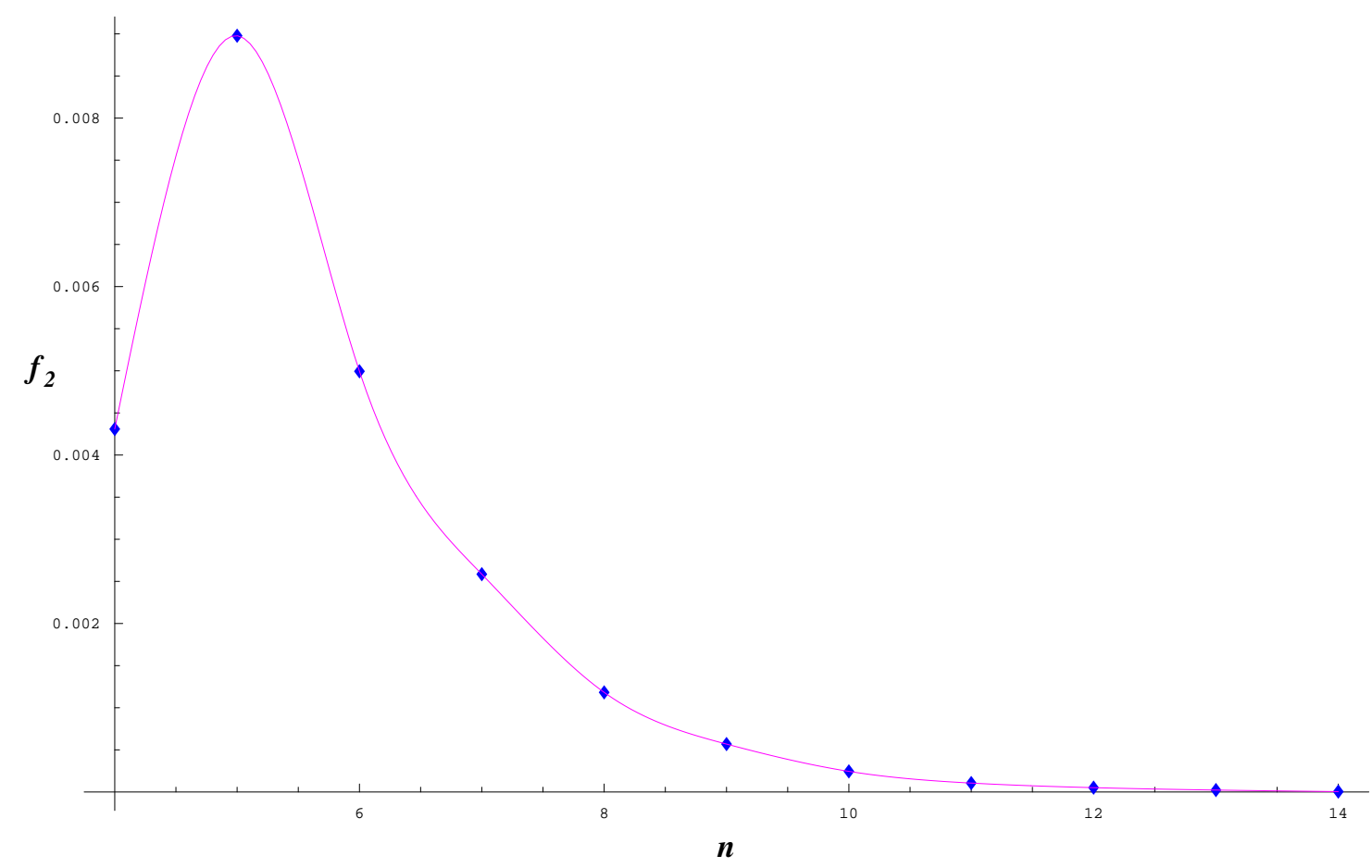

Figure 5.2: Fraction $f_{2}$ of the critical area of the lens under which maximal lensing is present, when the position of the central mass is perturbed. Instability increases with the number of lenses $n$. Fractions are in percentage units of the area of the critical disk.

given by

$$
f_{3} \equiv \frac{A_{\text {ellipse }}}{A_{r_{c r}}}=\frac{\Delta x \Delta y}{4 r_{c r}^{2}} .
$$

From the simulations, the maximum value for $f_{3}$ is $0.007 \%$ at $n=5$, with an exponential decrease with increasing $n$. Thus, the system is highly unstable to perturbations in the position of the source, requiring a good alignment between the center of the polygon, the source and the observer. The behaviour of $f_{3}$ as a function of $n$ is illustrated in Figure 5.3.

Lastly, to test the stability of the configuration under changes in the mass of one of the point lenses located at a vertex of the polygon, one of these lenses had its mass perturbed away from the value $m=\frac{1}{n-1}$, that had been set as the original value in the configuration. ${ }^{1}$ Through the simulations we find the range of values in the mass that keep the number of images maximum. This in turn is used to calculate $\Delta m$ as the size

\footnotetext{
${ }^{1}$ The normalization for the total mass in the $n-1$ lenses in the polygon is $M=1$ (see Section 3.1).
} 


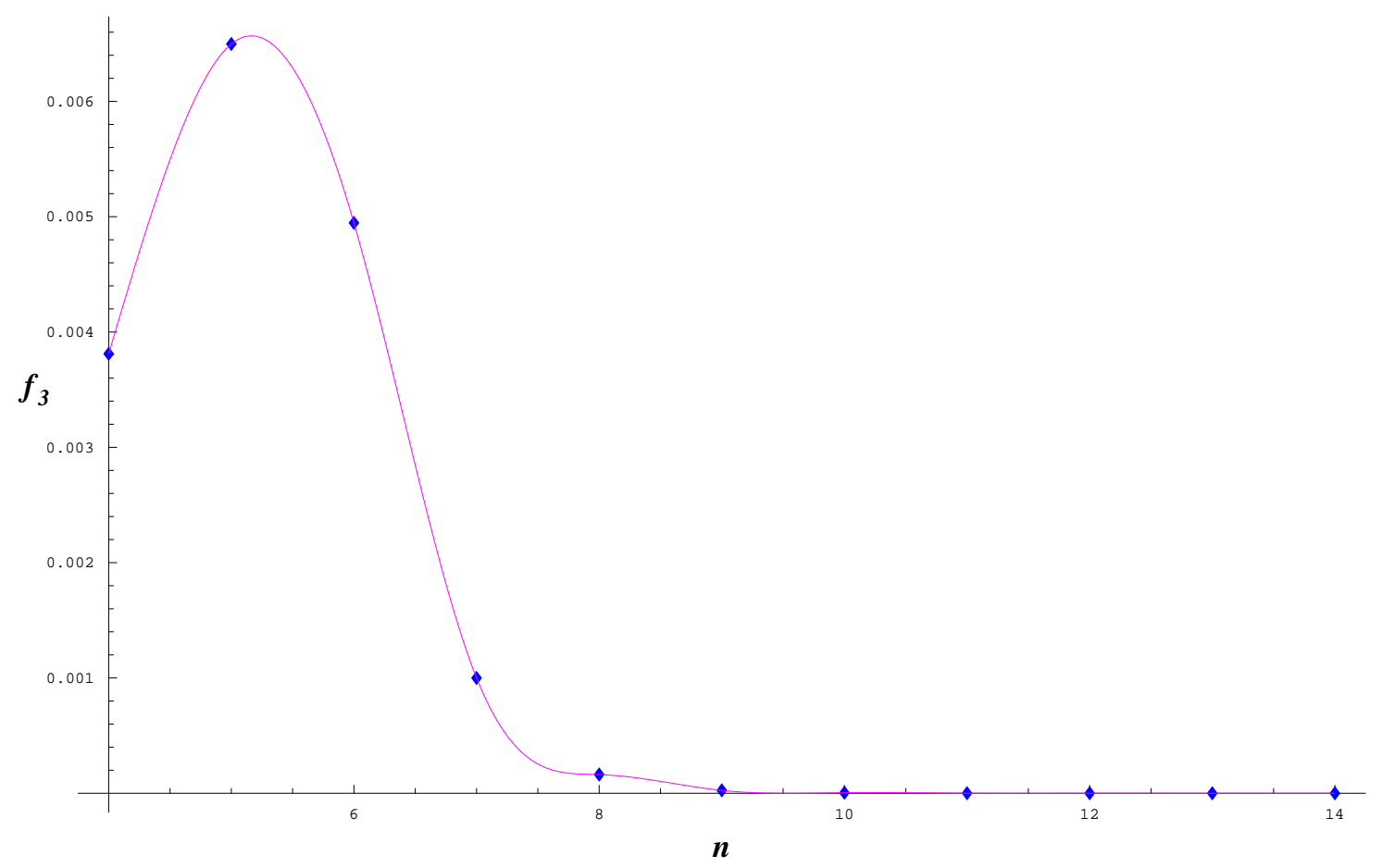

Figure 5.3: Fraction $f_{3}$ of the critical area of the lens under which maximal lensing is present, when the position of the source is perturbed. Instability increases with the number of lenses $n$. Fractions are in percentage units of the area of the critical disk.

of the interval in the mass available for maximal lensing. Of interest then, is the fraction that this mass represents of the initial value $m$ and is given by

$$
f_{4}=(n-1) \Delta m
$$

From the simulations, the maximum value for $f_{4}$ is $1.7 \%$ at $n=6$, with a fast decrease with increasing $n$. The behaviour of $f_{4}$ as a function of $n$ is illustrated in Figure 5.4. Altogether, the system is more stable under this perturbation to the mass of a lens than to any other perturbations in the configuration. Given that the bending angle is proportional to the product of the mass of the lens and the distance factor, a change in the mass is equivalent to a change in the distance factor. Furthermore, as will be illustrated in the next section, this stability in the mass translates into a stability of the system to changes in the distance from the observer to the lens. Thus, it is possible for these maximal lensing symmetric configurations to appear in systems of lenses that are 


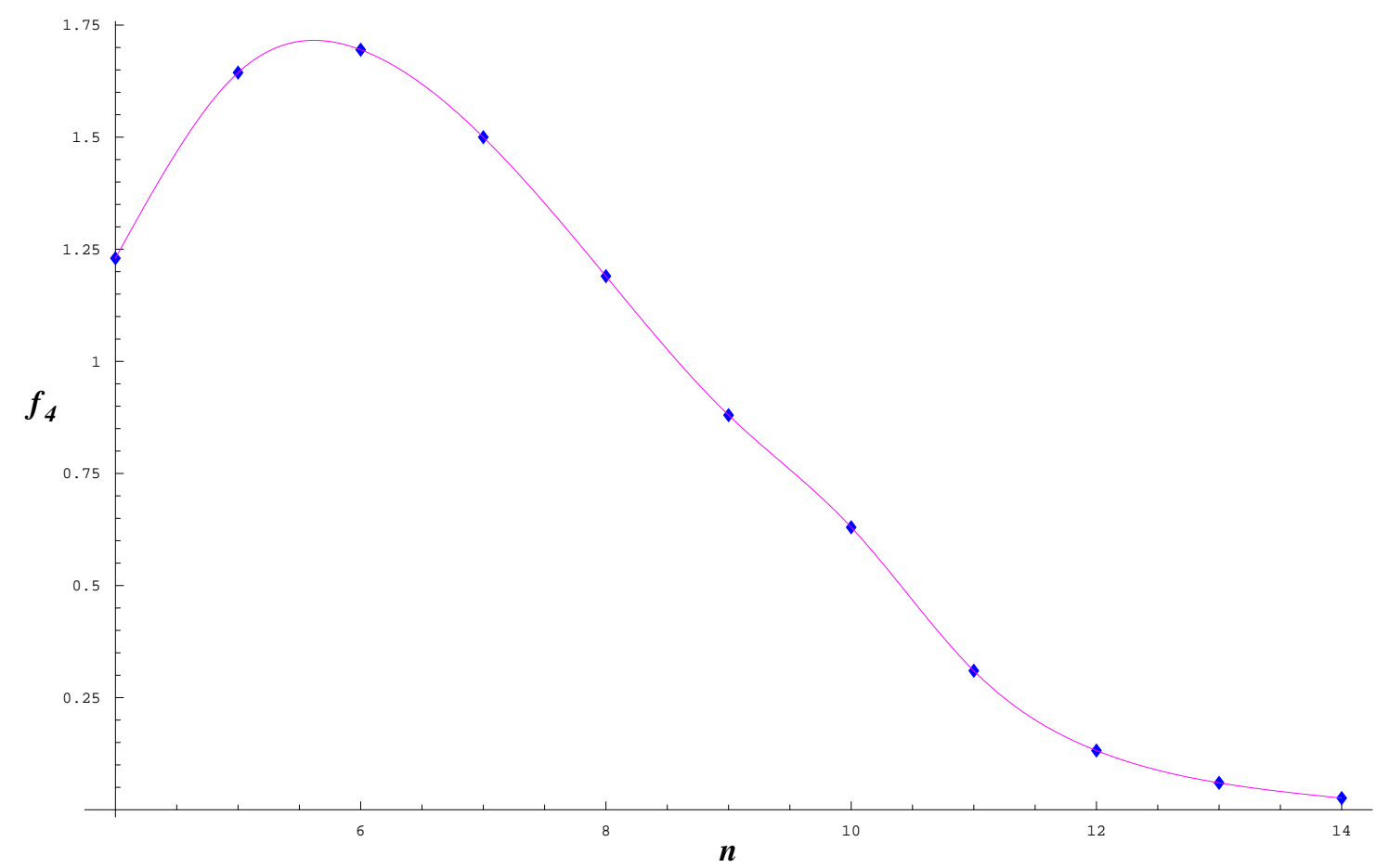

Figure 5.4: Fraction $f_{4}$ of the mass of one of the lenses on the polygon under which maximal lensing is present, when the value of the mass is perturbed. Instability increases with the number of lenses. Fractions are in percentage units of the mass of a lens $m=\frac{1}{n-1}$.

not gravitationally bound.

The results for the perturbations performed on the different variables for the maximal lensing case of a symmetric configuration with a small central mass, illustrate how these configurations are largely unstable under changes in the positions of lensing masses and source on the lens plane. However, due to the relative stability under perturbations in the mass of the lenses, we can conclude that the distances from the observer to the various lensing components of the gravitational array are not as constrained as other relevant quantities. This last statement follows from the observation that the net bending angle does not depend on the mass alone but on the product of the mass and the distance factor, as can be see in equations (1.46) and (2.3). This dependence will be used in the next section in order to study an application of these results to the search of small mass companions to stars in multiple star systems. 


\subsection{Applications of the Symmetric Configurations}

The sensitivity to the mass present at the center for the maximal lensing configuration described in Chapter 3, can be used to transform these theoretical results into a tool for detecting relatively small masses.

Consider a scenario with three equal masses arranged at the vertices of an equilateral triangle and a fourth small mass located at the center of the triangle. Maximal lensing requires the distance from a mass at a vertex to the center of the triangle to be less than the critical value in equation (3.1). For the configuration under study, this implies $r_{c r}=\frac{2^{1 / 3}}{\sqrt{3}} \cong 0.7274$. Given that $r<r_{c r}$ is a condition for maximal lensing, an upper bound on $m_{\star}$ is obtained:

$$
m_{\star}<r_{c r}^{2\left(\frac{9}{3}\right)}-\frac{r_{c r}^{6}}{1+r_{c r}^{6}} \cong 0.02
$$

As an example, if the lenses in the triangle are stars with a total mass $M=3.5 \mathrm{M}_{\odot}$, then $m_{\star}<0.07 \mathrm{M}_{\odot}$, making this lensing configuration sensitive to brown dwarfs and planets but not to more massive stars. ${ }^{2}$

We performed simulations perturbing the mass of one of the lenses at a vertex of the triangle to test the stability of the configuration (see Section B.2). We chose a value of $r=\frac{1}{2}$ for the radius of the triangle and $m_{c}=10^{-3}$ for the central mass perturbation, a value that corresponds roughly to 3.5 times the mass of Jupiter. This configuration of lenses in an equilateral triangle with a fourth central lens is illustrated in Figure 5.5, and a close-up of the central region can be found in Figure 5.6.

We were able to determine that the maximum number of images is obtained, as long as the perturbation to the mass at a vertex remains between an increase of $1.24 \%$ and a decrease of $2.74 \%$ around the value for the symmetric case of $m=\frac{1}{3}$. Since the bending

\footnotetext{
${ }^{2}$ The limit mass for a planet is roughly $13 \mathrm{M}_{\mathrm{J}} \approx 0.012 \mathrm{M}_{\odot}$ and the limit mass for a brown dwarf is approximately $75 \mathrm{M}_{\mathrm{J}} \approx 0.071 \mathrm{M}_{\odot}($ see Boss, 2001)
} 


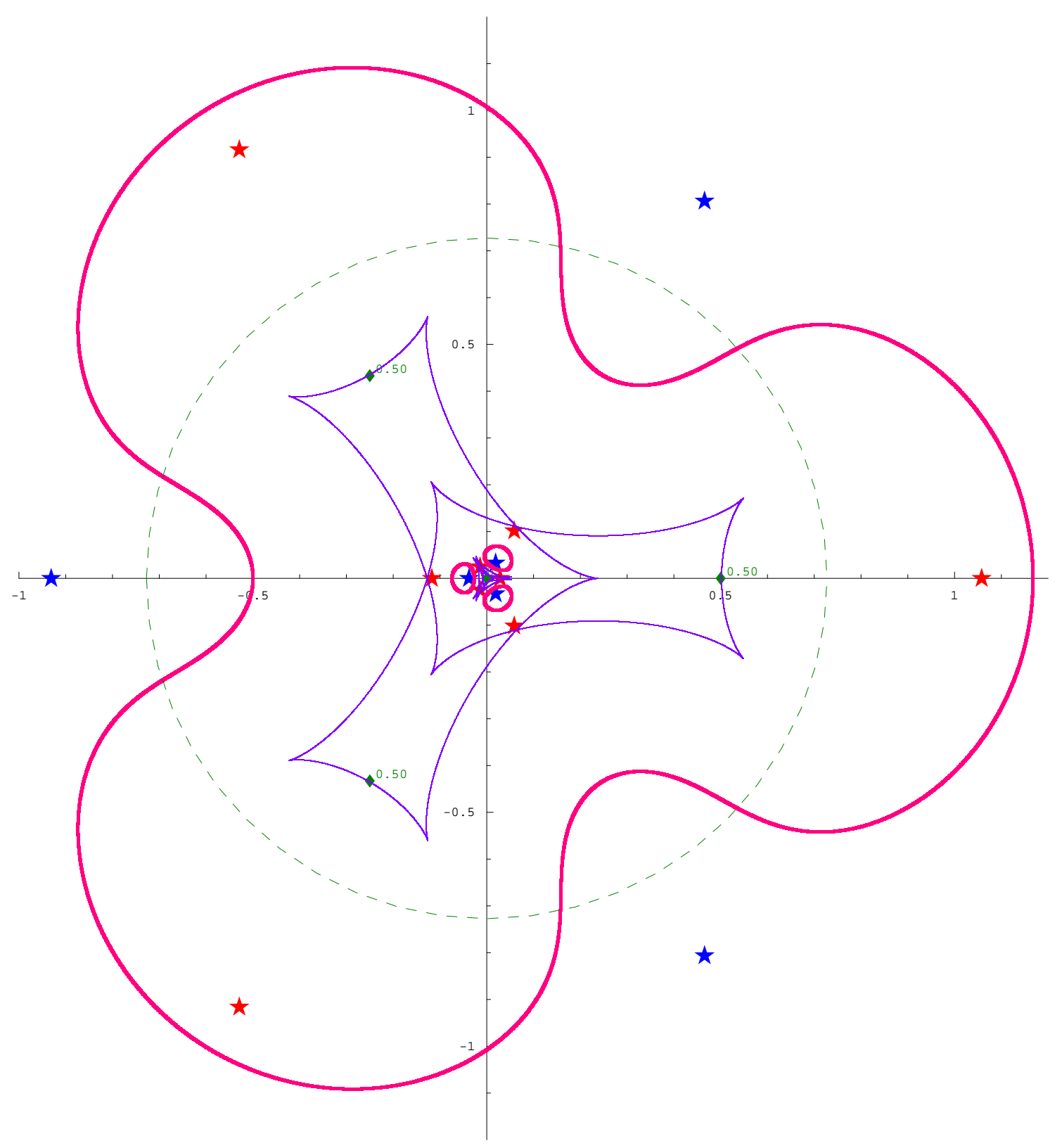

Figure 5.5: Central mass of $m_{c}=10^{-3}$ on a maximal lensing configuration of $n=3$ point-mass lenses at the vertices of an equilateral triangle. The dashed line represents the circle of radius $r_{c r} \cong 0.73$. Lenses at the vertices of the triangle, indicated with diamonds, are located at $r=0.50<r_{c r}$. Images are represented by five-pointed stars, while thick smooth lines represent critical curves and thin cuspy lines represent the caustic curves. 


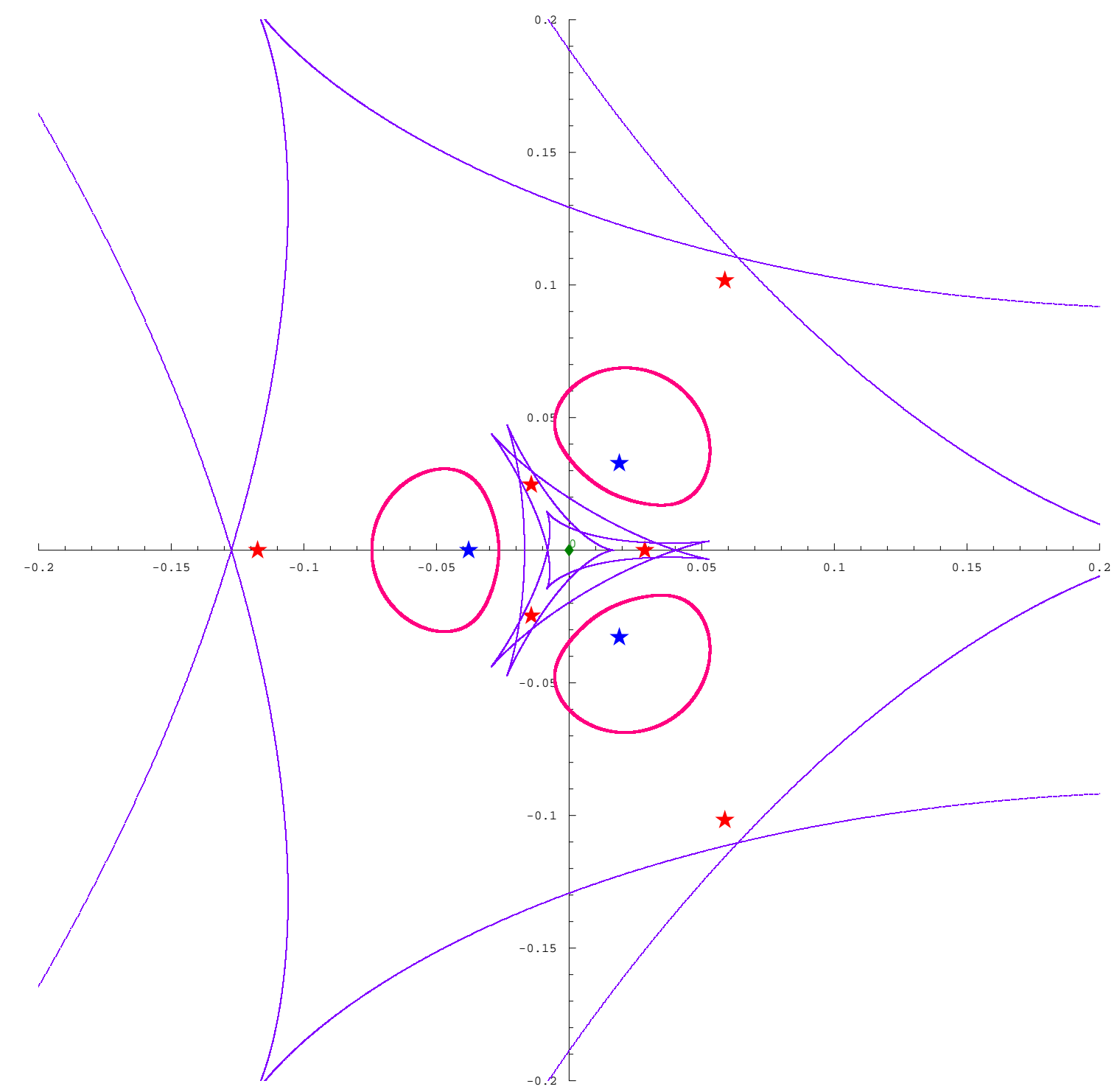

Figure 5.6: Detail on the central region of a maximal lensing configuration of $n=3$ point-mass lenses at the vertices of an equilateral triangle, with a small central mass of $m_{c}=10^{-3}$, indicated by a diamond. Images are represented by five-pointed stars, while thick smooth lines represent critical curves and thin cuspy lines represent the caustic curves. 


\begin{tabular}{|c|c|c|c|c|}
\hline$D_{O L}$ & $D_{O S}$ & $r=R_{E} / 2$ & $D_{-}$ & $D_{+}$ \\
\hline \hline $0.3 \mathrm{kpc}$ & $0.9 \mathrm{kpc}$ & $1.2 \mathrm{au}$ & $7.2 \mathrm{pc}$ & $18 \mathrm{pc}$ \\
\hline $6.5 \mathrm{kpc}$ & $8.5 \mathrm{kpc}$ & $3.3 \mathrm{au}$ & $83 \mathrm{pc}$ & $35 \mathrm{pc}$ \\
\hline $5.0 \mathrm{kpc}$ & $15 \mathrm{kpc}$ & $4.9 \mathrm{au}$ & $120 \mathrm{pc}$ & $300 \mathrm{pc}$ \\
\hline
\end{tabular}

Table 5.1: Perturbations on the distance to one of the lenses, in a maximal lensing configuration of identical point-mass lenses located at the vertices of an equilateral triangle with a small central lens. $D_{-}\left(D_{+}\right)$represents how much closer to (farther from) the observer one of the lenses can be moved, while maintaining the maximum number of images.

angle depends on the product of the lensing mass with the distance factor, it follows that this perturbations to the mass can also be modeled by perturbations to the distance between observer and lens.

In order to translate these results into astrophysically relevant quantities, dimensions must be recovered in the lens equation (3.3). As previously noted in Section 3.1, the Einstein radius $R_{E}$ of the total mass for the lenses in the polygon is used as the normalization factor for distances on the lens plane (see also equation 2.11). Therefore, setting $R_{E}=1$ implies $\sqrt{D \Phi M}=1$, where $M$ is the total mass of the deflectors at the vertices of the regular polygon, $\Phi \equiv \frac{4 G}{c^{2}} \cong 1.91 \times 10^{-13} \mathrm{pc} / \mathrm{M}_{\odot}$, and $D$ is the distance factor as defined in (2.2).

Given values for the distance to the lensing configuration $D_{O L}$ and for the distance to the source $D_{O S}$, it is then possible to calculate the radius of the lensing configuration $r=\frac{R_{E}}{2}$, representing the distance from the stars to the small central mass. Choices for $D_{O L}$, when combined with the perturbations on the mass in one of the stars in the triangle, lead then to allowed perturbations in the distance of one of these stars with respect to the observer. That is, values for how closer or farther from the observer one of the stars can be located, while the configuration is still a maximal lensing scenario. For the example of three stars at the vertices of an equilateral triangle with total mass $M=3.5 \mathrm{M}_{\odot}$ and a small mass $m_{c}=3.5 \mathrm{M}_{\mathrm{J}}$ at the center, we have summarized the results of the perturbations in Table 5.1, for three different sets of choices for $D_{O L}$ and $D_{O S}$. The values of $D_{-}$and $D_{+}$represent the distance that one of the stars can be moved 
closer to or farther from the observer, with respect to the lens plane at $D_{O L}$, while maintaining the maximum number of images. Thus, the information on the stability of these configurations under perturbations to the distance between the observer and one of the stars, can be found in the values of $D_{-}$and $D_{+}$. Thus we can conclude from the values found and reported in Table 5.1, that the system of stars does not need to be a gravitationally bound group in order to exhibit maximal lensing. Therefore, the likelihood of this type of scenario to be observed increases.

The angular separations of the produced images in the maximal lensing configurations discussed in this section are in the order of the micro-arcseconds or less and thus the resolution of separate images in such a system cannot be achieved with currently available technologies. However, as it is done in microlensing, the light curves of lensing events that lead to maximal lensing configurations will then yield information about the system. The richness of the caustic curve structure for the maximal lensing configurations leads to magnification curves with specific signatures that can be studied to determine properties of the system.

We performed microlensing simulations on the system described at the beginning of this section. As illustrated in Figure 5.5, three identical point-mass lenses are located at the vertices of an equilateral triangle, fixed at a distance $r=\frac{1}{2}$ in units of the Einstein radius $R_{E}$. As previously discussed, when a small point mass is added at the center of this configuration, the maximum number of images is produced. The passage of this small mass close to the center of the configuration will induce the crossing of multiple caustic curves by the source and thus changes in the total magnification of the images produced.

The mass in the configuration is normalized so that the total mass of the three lenses at the vertices of the triangle is $M=1$, while as in previous simulations, we chose the mass of the central lens to be $m_{c}=10^{-3}$. These choices of mass perturbation and radius of the triangle lead to a maximal lensing scenario when the small mass perturbation 
passes by the center of the configuration. Samples of microlensing light curves can thus be obtained by allowing the mass perturbation to move across the lens plane while the total magnification for all images is calculated as a function of the mass position.

Given that the magnifications produced by these systems can be of the order of a few thousands, the features of these light curves are more easily observed in the logarithmic scale of magnitudes. If the total magnification of the images in a given configuration has a value $\mu$, then it will correspond to a magnitude change $\Delta m$ over the magnitude of the unlensed source given by

$$
\Delta m=2.5 \log \mu .
$$

If the position of the small mass perturbation is given by $z_{c}=x_{c}+i y_{c}$, we have illustrated in Figure 5.7 the light curve for a central mass with fixed horizontal position $x_{c}=0$ and moving vertically according to $-0.16<y_{c}<0.16$. A detail of the central region of this microlensing light curve is in Figure 5.8. Given that the mass perturbation passes by the center of the configuration, the maximum number of images is realized.

A different microlensing scenario can be found in Figure 5.9, where the mass perturbation transits off-center and the number of images is $N=13$, two less than the maximum. In this light curve, the mass perturbation has a fixed horizontal position $x_{c}=\frac{1}{100}$, and it moves vertically according to $-0.1<y_{c}<0.1$. Further detail on the central region of this light curve can be found in Figure 5.10.

It is worth noting that even though simulations done with point sources produce formally diverging magnifications at the crossing of the caustic lines, this problem can be fixed through the use of finite-sized sources.

\subsection{Asymmetric Configurations}

In Section 5.1, on the symmetric configuration with a central mass perturbation as a maximal lensing scenario, we illustrate how unstable these configurations are and thus 


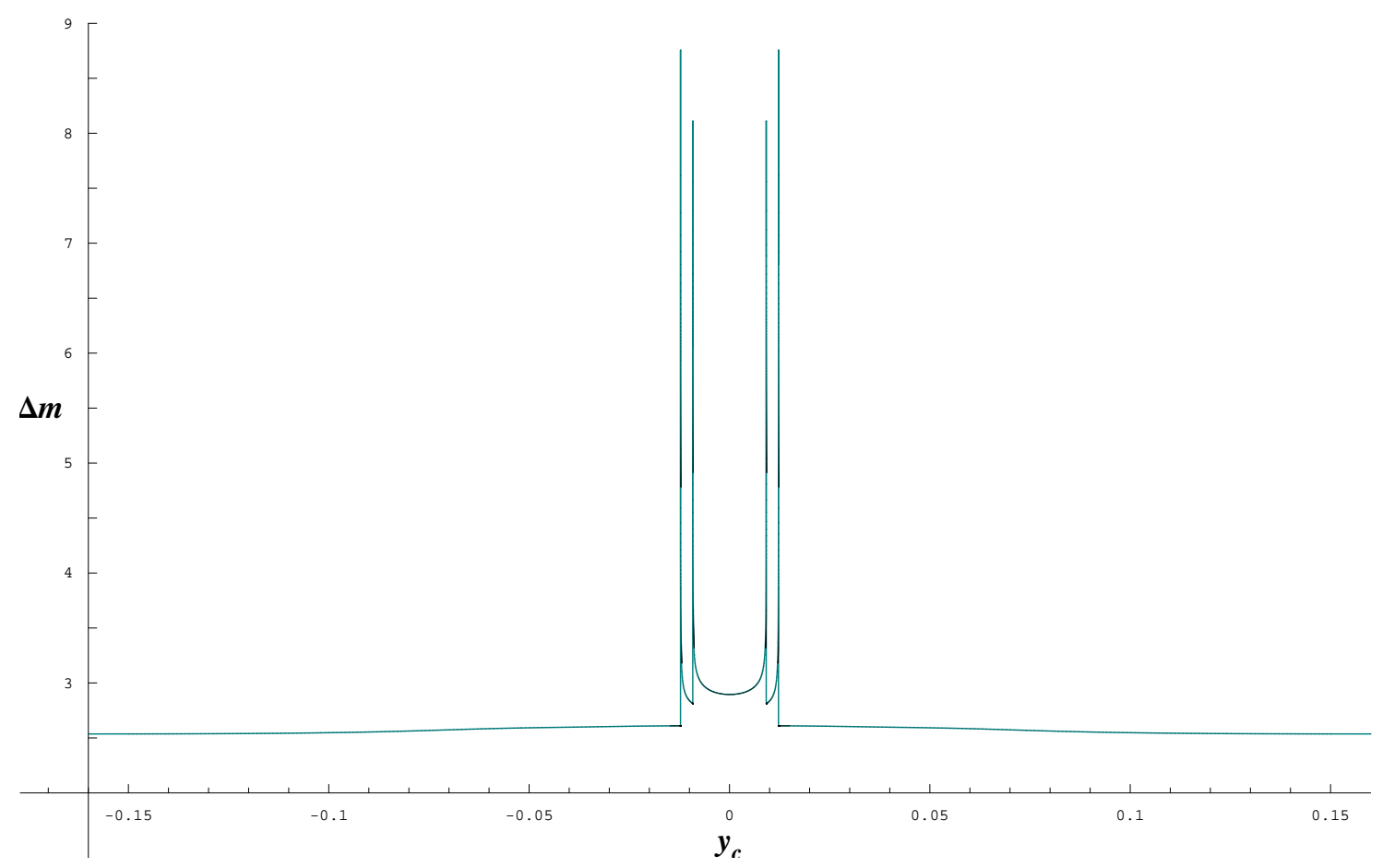

Figure 5.7: Total magnitude change $\Delta m$ in a configuration of $n=3$ point-mass lenses at the vertices of an equilateral triangle, when a small mass $m_{c}$ transits through the center of the system. The mass in transit is located at $z_{c}=x_{c}+i y_{c}$ with $x_{c}=0$.

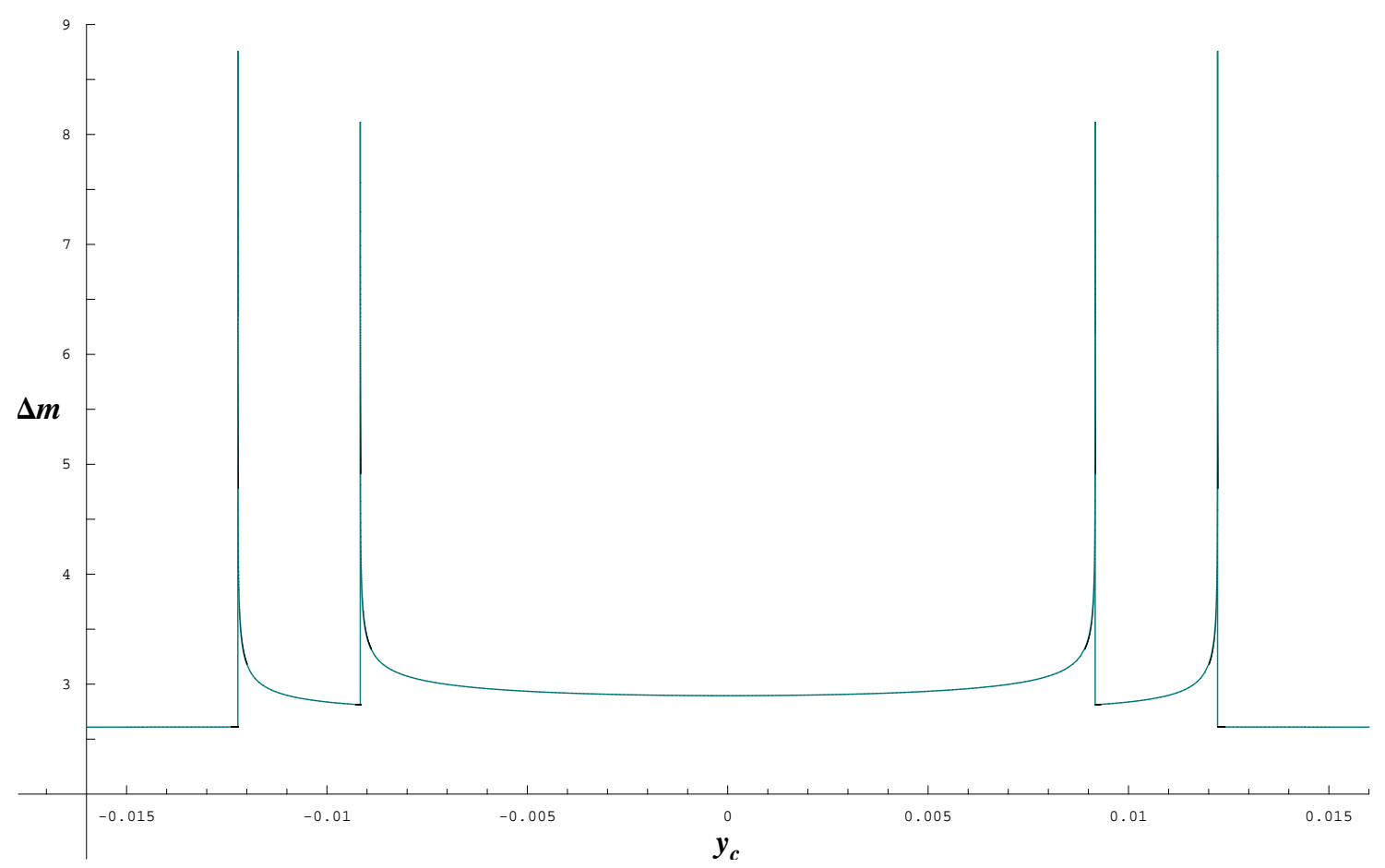

Figure 5.8: Detail on the total magnitude change $\Delta m$ in a configuration of $n=3$ pointmass lenses at the vertices of an equilateral triangle, when a small mass $m_{c}$ transits through the center of the system. The mass in transit is located at $z_{c}=x_{c}+i y_{c}$ with $x_{c}=0$. 


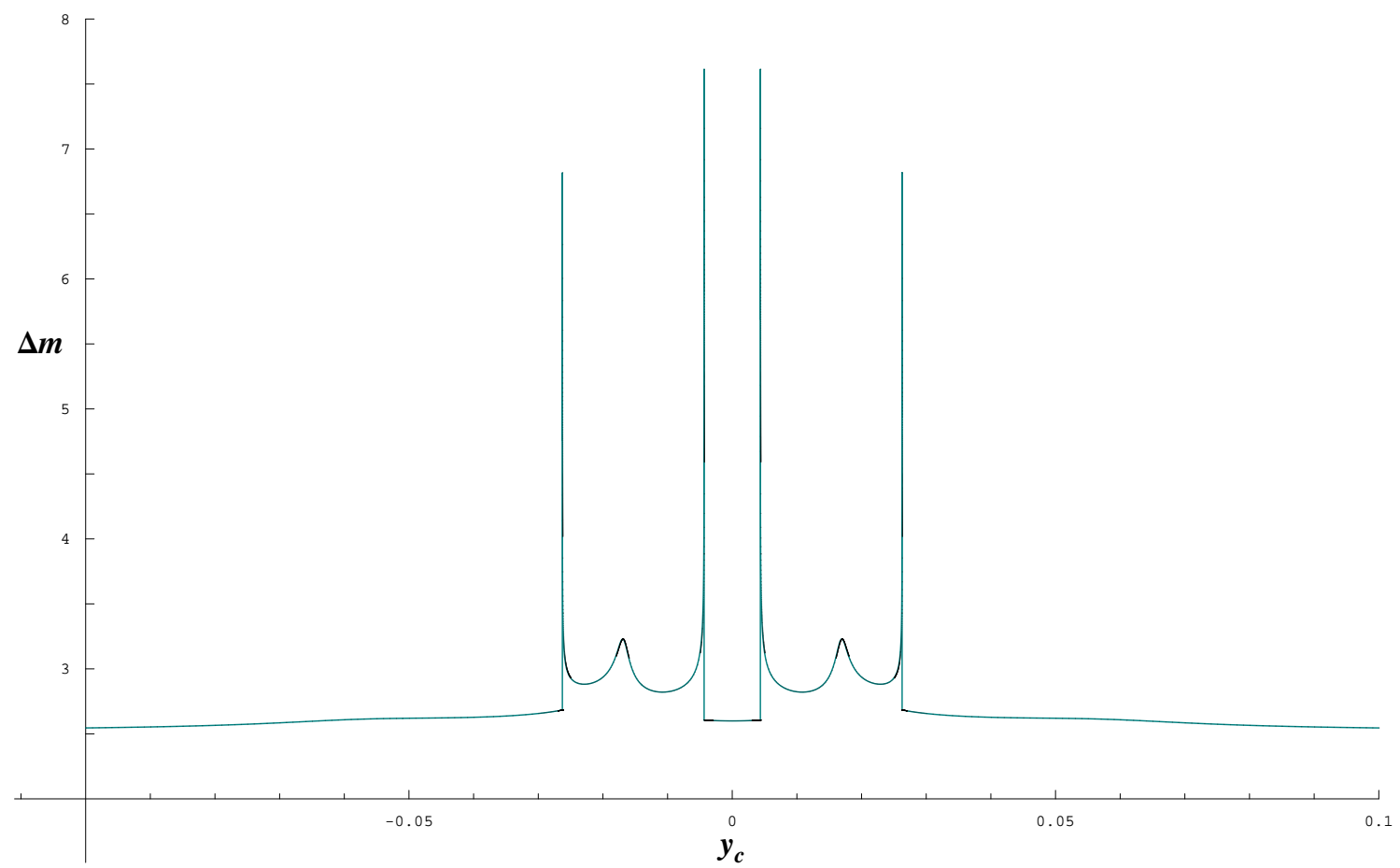

Figure 5.9: Total magnitude change $\Delta m$ in a configuration of $n=3$ point-mass lenses at the vertices of an equilateral triangle, when a small mass $m_{c}$ transits off the center of the system. The mass in transit is located at $z_{c}=x_{c}+i y_{c}$ with $x_{c}=\frac{1}{100}$.

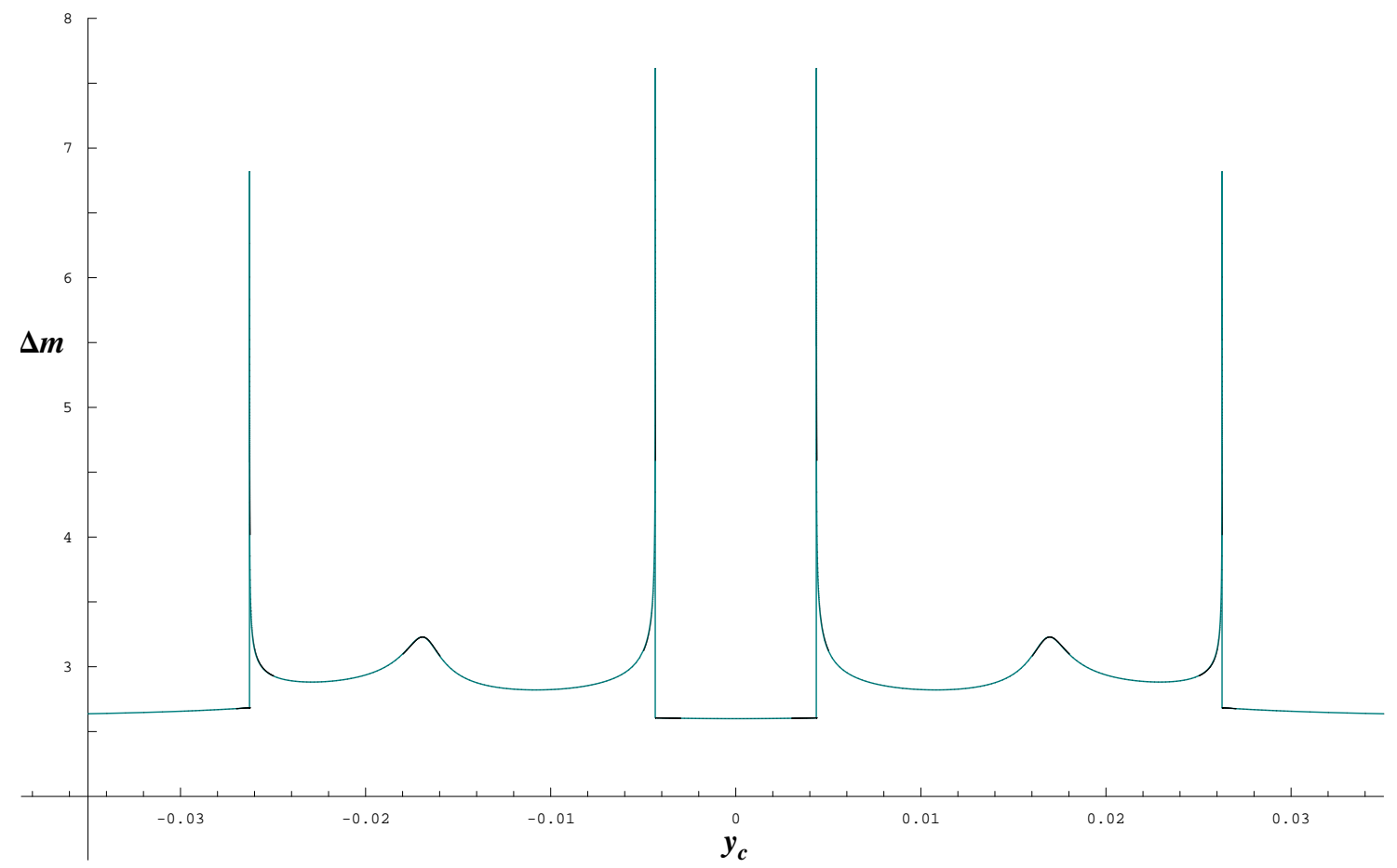

Figure 5.10: Detail on the total magnitude change $\Delta m$ in a configuration of $n=3$ pointmass lenses at the vertices of an equilateral triangle, when a small mass $m_{c}$ transits off the center of the system. The mass in transit is located at $z_{c}=x_{c}+i y_{c}$ with $x_{c}=\frac{1}{100}$. 
remark that the probability of direct observation is quite low, except for the lowest values of the number of lenses. ${ }^{3}$

The question then arises as to whether or not maximal lensing configurations that do not require the symmetry with a central perturbation are possible. We call these systems Asymmetric Configurations.

Our starting point is a special lensing configuration for $n=4$ point-mass lenses previously described in Section 2.3 and originally studied in Rhie (2001). In his paper, Rhie describes a maximal lensing configuration of 4 identical point-mass lenses producing a total of $N=15$ images. ${ }^{4}$ However, this configuration is not for three lenses in an equilateral triangle with a smaller mass at the center of the triangle, like those described in Chapter 3. Instead, the system of lenses, each of mass $m=\frac{1}{4}$, is arranged asymmetrically with the positions of the lenses given by $z_{1}=(0.8,0), z_{2}=(0,0.5), z_{3}=(-0.8,0)$ and $z_{4}=(0,-0.5)$

Furthermore, even though the symmetry has been broken away from the square, lenses remain along lines separated by $90^{\circ}$ and are paired at equal distances from the origin, so that $\left|z_{1}\right|=\left|z_{3}\right|$ and $\left|z_{2}\right|=\left|z_{4}\right|$. Maintaining these symmetries, we ran simulations to find more asymmetric maximal lensing configurations (see Section B.3).

From the simulations we find that the most stable configuration under changes in the positions of the lenses is described by point masses located at $z_{1}=(0.85,0), z_{2}=(0,0.45)$, $z_{3}=(-0.85,0)$ and $z_{4}=(0,-0.45)$. Illustrated in Figure 5.11, this maximal lensing configuration will be used in the next section to study the stability of asymmetric maximal lensing configurations.

The existence of asymmetric maximal lensing configurations for $n=4$ identical lenses

\footnotetext{
${ }^{3}$ Configurations of lenses in a triangle, a square and a pentagon with a small central lens are the most stable (Section 5.1). The systems are quite sensitive to changes in positions on the lens plane, but stable under mass perturbations, and thus stable under perturbations on the distance to the observer.

${ }^{4}$ As previously noted in Section 2.3, the study of this configuration by Rhie (2001) provided the first proof that the maximum number of images was not the upper bound of $n^{2}+1$ found by Witt (1990) or the $3 n+1$ from the symmetric configuration studied by Mao et al. (1999).
} 


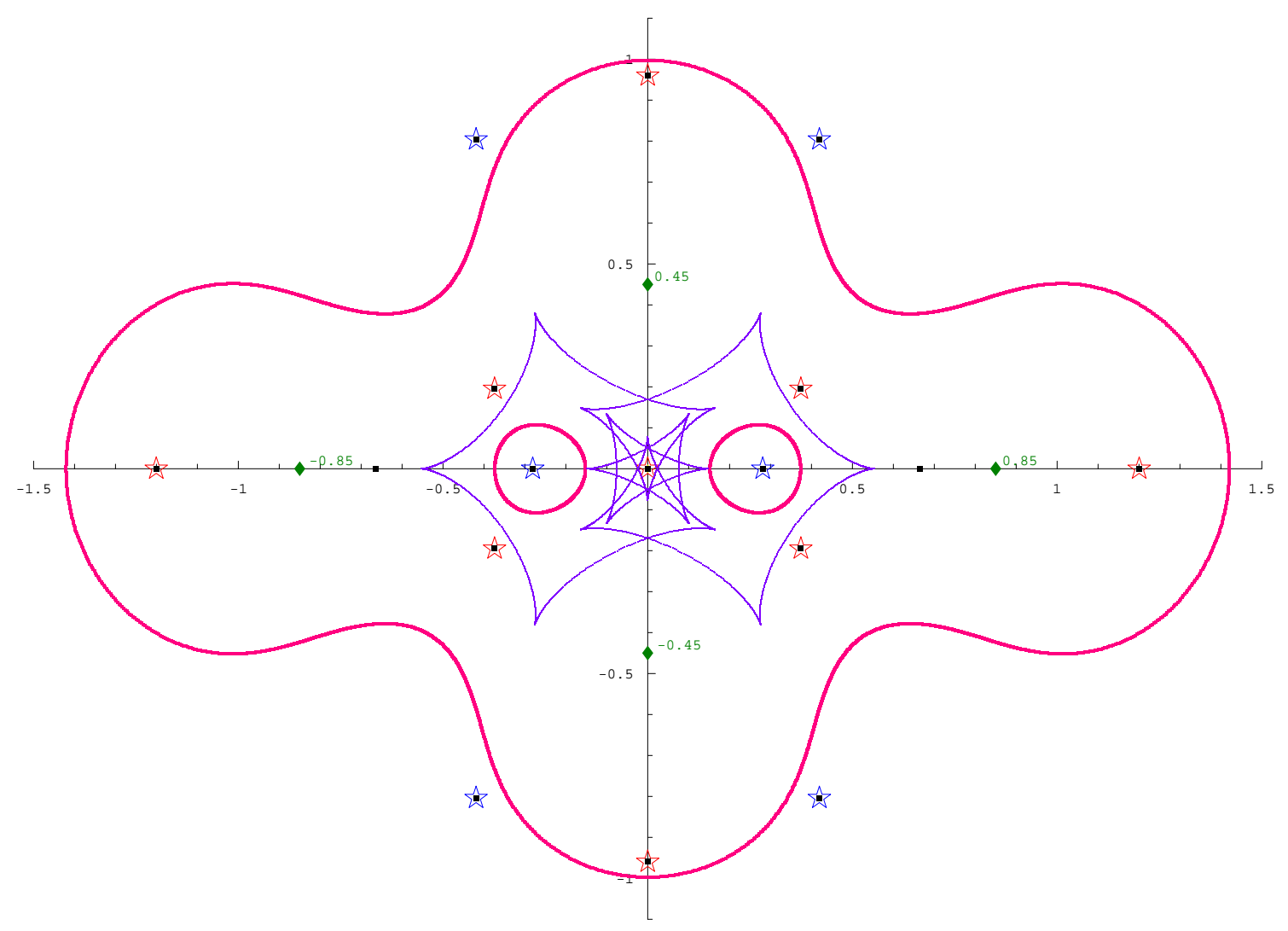

Figure 5.11: Asymmetric maximal lensing configuration of $n=4$ point-mass lenses. Solutions to Witt's polynomial are marked by small squares, images are represented by five-pointed stars and lenses, indicated with diamonds, are labeled with their distances to the center. Thick smooth lines are the critical curves and thin cuspy lines are the caustics. 
motivated our search for similar configurations of different values of $n$. We will then consider systems of $n$ identical point-mass lenses with normalized total mass $M=1$, so that each lens has mass $m=\frac{1}{n}$.

To study the case of $n=5$ lenses, we keep the point-mass deflectors along the vertex lines of a regular pentagon. That is, lenses remain along lines separated by $72^{\circ}$. In a similar fashion to the asymmetric configuration for $n=4$, some of the lenses are paired to have the same distance to the origin, so that when going around counter-clockwise from the first mass at $z_{1}$ to the last mass at $z_{5}$, the distances from the center obey the relationships $\left|z_{2}\right|=\left|z_{5}\right|$ and $\left|z_{3}\right|=\left|z_{4}\right|$.

Details of the simulations can be found in Section B.3, and illustrated in Figure 5.12 is an asymmetric maximal lensing configuration for $n=5$. The positions of the lenses are given in polar coordinates by $z_{1}=\left(0.50,0^{\circ}\right), z_{2}=\left(0.90,72^{\circ}\right), z_{3}=\left(0.53,144^{\circ}\right)$, $z_{4}=\left(0.53,-144^{\circ}\right)$ and $z_{5}=\left(0.90,-72^{\circ}\right)$.

Similarly, to study the case of $n=6$ lenses, we keep the point-mass deflectors along the vertex lines of a regular hexagon. That is, lenses will remain along lines separated by $60^{\circ}$. Different arrangements were made in which sets of lenses were paired to have the same distance to the origin. When going around counter-clockwise from the first mass at $z_{1}$ to the last mass at $z_{6}$, the distances from the center were set to obey the relationships $\left|z_{1}\right|=\left|z_{4}\right|,\left|z_{2}\right|=\left|z_{6}\right|$ and $\left|z_{3}\right|=\left|z_{5}\right|$. Another alternative pairing used in the simulations has the distances from the center set according to $\left|z_{1}\right|=\left|z_{4}\right|,\left|z_{2}\right|=\left|z_{5}\right|$ and $\left|z_{3}\right|=\left|z_{6}\right|$.

Details of the simulations can be found in Section B.3. These simulations did not yield a maximal lensing configuration up to the precision used in the algorithms. For the best simulations, instead of $N=25$ images that are known to be possible for $n=6$ lenses, we obtained several configurations with $N=21$ images. $^{5}$

Illustrated in Figure 5.13 is one of the configurations found to have $N=21$ images for

\footnotetext{
${ }^{5}$ This is already more than the $3 n+1=19$ from the symmetric configuration studied by Mao et al. (1999), a reminder that maximal lensing requires some symmetry in order to maximize the gravitational effects but not so much that degenerate solutions appear (see Rhie, 2001).
} 


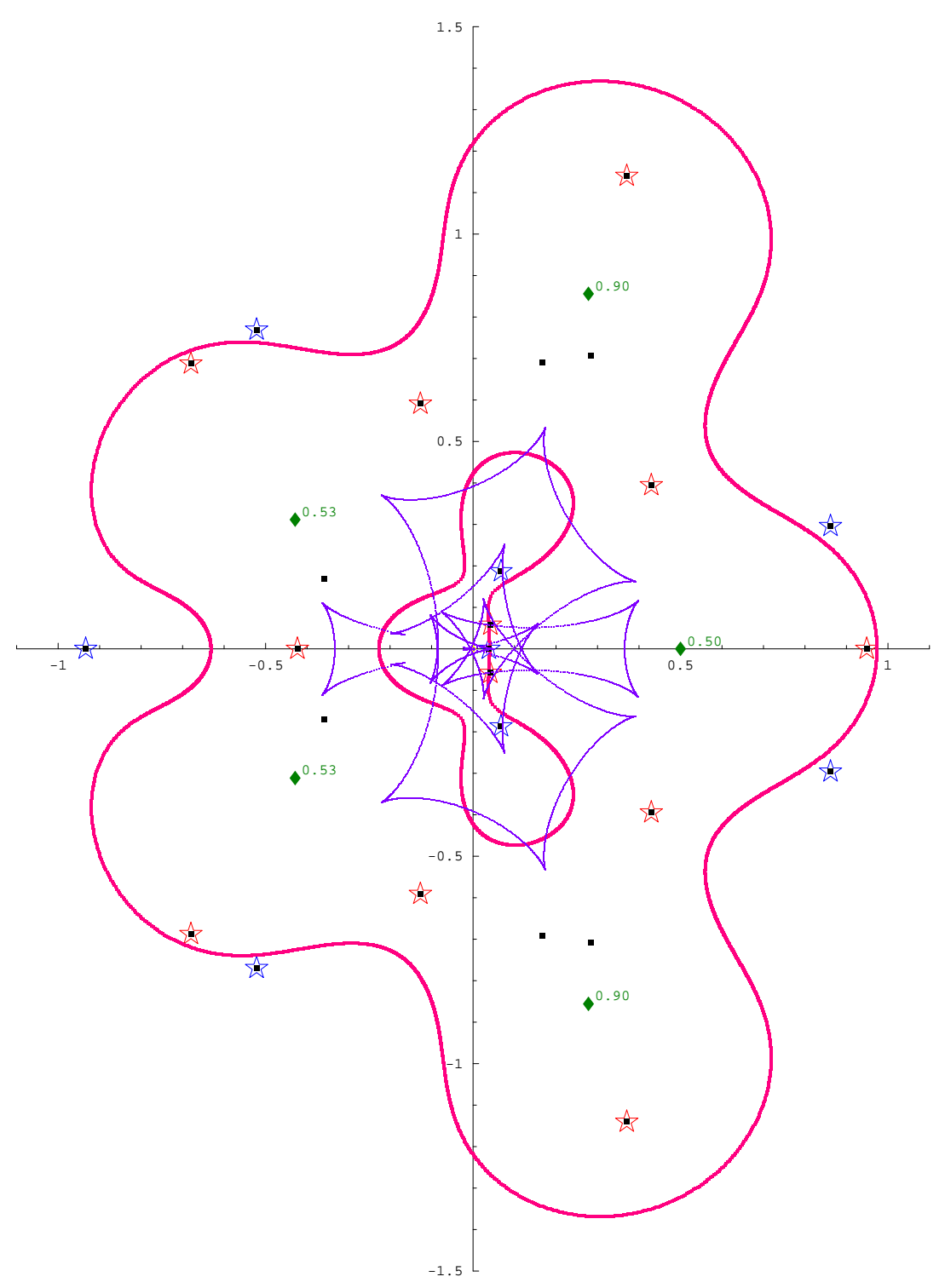

Figure 5.12: Asymmetric maximal lensing configuration of $n=5$ point-mass lenses. Solutions to Witt's polynomial are marked by small squares, images are represented by five-pointed stars and lenses, indicated with diamonds, are labeled with their distances to the center. Thick smooth lines are the critical curves and thin cuspy lines are the caustics. 
$n=6$. The positions of the lenses are given in polar coordinates by $z_{1}=\left(0.65,0^{\circ}\right), z_{2}=$ $\left(0.35,60^{\circ}\right), z_{3}=\left(0.85,120^{\circ}\right), z_{4}=\left(0.65,180^{\circ}\right), z_{5}=\left(0.85,-120^{\circ}\right)$ and $z_{6}=\left(0.35,-60^{\circ}\right)$.

\subsection{Perturbations on Asymmetric Configurations}

In this section we present results on the stability of the maximal lensing asymmetric point lens configurations described in Section 5.3 for $n=4$ and $n=5$ lenses. Information on the simulations can be found in Section B.4.

For $n=4$ we start with a configuration of lenses each of mass $m=\frac{1}{4}$. The positions of the lenses are given by $z_{1}=(0.85,0), z_{2}=(0,0.45), z_{1}=(-0.85,0)$ and $z_{4}=(0,-0.45)$ on the lens plane. ${ }^{6}$ This configuration is illustrated in Figure 5.11.

The quantities perturbed were: the position of the lenses at $z_{1}$ and $z_{2}$ along radial and tangential directions, the mass of the lenses at $z_{1}$ and $z_{2}$, and the position of the source along the horizontal and vertical directions. Note that given the symmetry of the configuration, perturbations on $z_{1}$ are equivalent to perturbations on $z_{3}$, and perturbations on $z_{2}$ are in turn equivalent to perturbations on $z_{4}$.

From perturbations in the positions of lenses and source we obtain ranges of values around the unperturbed configuration that produce the maximum number of images. These ranges in positions are in turn used to calculate the areas of ellipses around the unperturbed system in a way similar to that described in Section 5.1. The values of the areas thus obtained can then be compared against the area of the Einstein disk to obtain a fraction indicative of the stability of the configuration on the lens plane.

The perturbations on the position of the lens located at $z_{1}$ result in a region of maximal lensing that is $1.12 \%$ of the area of the Einstein disk. Similarly, the perturbations on the position of the lens located at $z_{2}$ produce a maximal lensing region that is $0.32 \%$ of the

\footnotetext{
${ }^{6}$ This particular configuration allows for the largest maximal lensing phase space, when the lenses are constrained to remain along the coordinate axes and distances to the origin paired according to $\left|z_{1}\right|=\left|z_{3}\right|$ and $\left|z_{2}\right|=\left|z_{4}\right|$.
} 


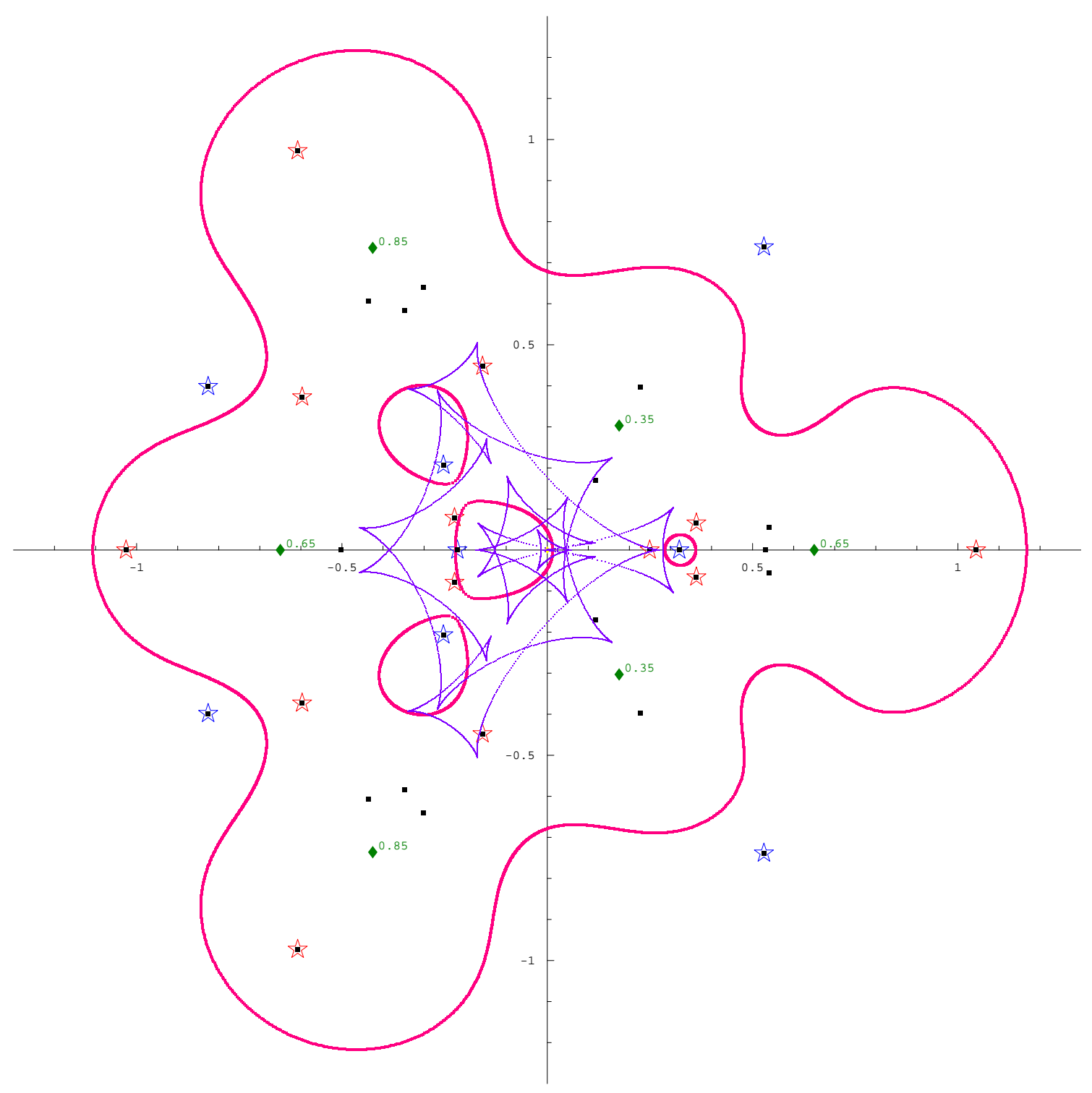

Figure 5.13: Asymmetric lensing configuration of $n=6$ point-mass lenses that produces $N=21$ images. Solutions to Witt's polynomial are marked by small squares, images are represented by five-pointed stars and lenses, indicated with diamonds, are labeled with their distances to the center. Thick smooth lines are the critical curves and thin cuspy lines are the caustics. 


\begin{tabular}{|c|c|c|c|}
\hline \multicolumn{2}{|c|}{ Asymmetric } & \multicolumn{2}{c|}{ Central Mass Symmetric } \\
\hline \hline Position $z_{1}$ & $1.12 \%$ & Position $z_{k}$ & $0.0012 \%$ \\
\hline Position $z_{2}$ & $0.32 \%$ & Position $z_{c}$ & $0.0043 \%$ \\
\hline Position $z_{s}$ & $0.12 \%$ & Position $z_{s}$ & $0.0038 \%$ \\
\hline Mass $\left(z_{1}, z_{2}\right)$ & $40.0 \%$ & Mass $z_{k}$ & $1.23 \%$ \\
\hline
\end{tabular}

Table 5.2: Stability comparison of the asymmetric configuration against the symmetric configuration with a central mass $\left(n=4\right.$ lenses). The source is represented by $z_{s}$, and the lenses in the asymmetric configuration are $z_{1}$ and $z_{2}$. In the central mass symmetric configuration, $z_{c}$ represents the central lens while $z_{k}$ is a lens at one of the vertices.

area of the Einstein disk. For the source, the perturbations on the position result in a maximal lensing region that is $0.12 \%$ of the area of the Einstein disk.

From the perturbations on the mass of the lenses at $z_{1}$ and at $z_{2}$ we obtain the ranges of values where maximal lensing occurs around the unperturbed mass of $m=\frac{1}{4}$. Maximal lensing is present as long as the mass is in the range [0.178, 0.278], a region that is $40 \%$ of the mass in one of the lenses.

It is now possible to compare the stability of this asymmetric configuration of $n=4$ lenses against the stability of the central mass configuration with the same number of lenses previously studied in Section 5.1. In Table 5.2, allowed perturbations for the position are given in terms of the area of the Einstein disk for the asymmetric configuration, and in terms of the area of the critical disk for the central mass symmetric configuration. In the same table, allowed perturbations for the mass are given in terms of the mass of one of the lenses. ${ }^{7}$ From Table 5.2 it is then evident that the asymmetric configuration is more stable than the central mass symmetric configuration for $n=4$ point-mass lenses. Therefore, an asymmetric maximal lensing system with $n=4$ deflectors is more likely to be observed.

For $n=5$ we start with a configuration of lenses each of mass $m=\frac{1}{5}$. The positions of the lenses are given by $z_{1}=\left(0.50,0^{\circ}\right), z_{2}=\left(0.90,72^{\circ}\right), z_{3}=\left(0.53,144^{\circ}\right), z_{4}=$ $\left(0.53,-144^{\circ}\right)$ and $z_{5}=\left(0.90,-72^{\circ}\right)$, when expressed in polar coordinates on the lens

\footnotetext{
${ }^{7}$ Perturbations on the mass of the central lens are excluded here as those were studied in deriving equation (3.9).
} 
plane. ${ }^{8}$ This configuration is illustrated in Figure 5.12.

The quantities perturbed were: the position of the lenses at $z_{1}, z_{2}$ and $z_{3}$ along radial and tangential directions, the mass of the lenses at $z_{1}, z_{2}$ and $z_{3}$, and the position of the source along the horizontal and vertical directions. Note that given the symmetry of the configuration, perturbations on $z_{2}$ are equivalent to perturbations on $z_{5}$, and perturbations on $z_{3}$ are in turn equivalent to perturbations on $z_{4}$.

From perturbations in the positions of lenses and source we obtain ranges of the values around the unperturbed configuration that produce the maximum number of images. These ranges in positions are in turn used to calculate the areas of ellipses around the unperturbed system in a way similar to that used in Section 5.1. The values of the areas thus obtained can then be compared against the area of the Einstein disk to obtain a fraction indicative of the stability of the configuration on the lens plane.

The perturbations on the position of the lens located at $z_{1}$ result in a region of maximal lensing that is $6.6 \times 10^{-7}$ of the area of the Einstein disk. Similarly, the perturbations on the position of the lens located at $z_{2}$ produce a maximal lensing region that is $1.5 \times 10^{-6}$ of the area of the Einstein disk and the perturbations on the position of the lens located at $z_{3}$ produce a maximal lensing region that is $1.3 \times 10^{-7}$ of the area of the Einstein disk. For the source, the perturbations on the position result in a maximal lensing region that is $7.3 \times 10^{-7}$ of the area of the Einstein disk.

From the perturbations on the mass of the lenses at $z_{1}, z_{2}$ and $z_{3}$ we obtain the ranges of values where maximal lensing occurs around the unperturbed mass of $m=\frac{1}{5}$. Maximal lensing is present in regions that are respectively $12.95 \%, 0.135 \%$ and $0.155 \%$ of the mass in one of the lenses.

It is now possible to compare the stability of this asymmetric configuration of $n=5$ lenses against the stability of the equivalent central mass configuration with the same

\footnotetext{
${ }^{8}$ This configuration was selected because the values of the distances to the origin are the midpoints of the ranges found in the asymmetric configurations with maximal lensing for $n=5$ lenses.
} 


\begin{tabular}{|c|c|c|c|}
\hline \multicolumn{2}{|c|}{ Asymmetric } & \multicolumn{2}{c|}{ Central Mass Symmetric } \\
\hline \hline Position $z_{1}$ & $0.000066 \%$ & \multirow{2}{*}{ Position $z_{k}$} & $0.0028 \%$ \\
Position $z_{2}$ & $0.00015 \%$ & \multirow{2}{*}{ Position $z_{c}$} & $0.0090 \%$ \\
\cline { 1 - 2 } Position $z_{3}$ & $0.000013 \%$ & & $0.0065 \%$ \\
\cline { 1 - 2 } Position $z_{s}$ & $0.000073 \%$ & Position $z_{s}$ & \multirow{2}{*}{ Mass $z_{k}$} \\
\cline { 1 - 2 } Mass $z_{1}$ & $12.95 \%$ & \\
\cline { 1 - 2 } Mass $z_{2}$ & $0.135 \%$ & & \\
\hline Mass $z_{3}$ & $0.155 \%$ & & \\
\hline
\end{tabular}

Table 5.3: Stability comparison of the asymmetric configuration against the symmetric configuration with a central mass ( $n=5$ lenses). The source is represented by $z_{s}$, and the lenses in the asymmetric configuration are $z_{1}, z_{2}$ and $z_{3}$. In the central mass symmetric configuration, $z_{c}$ represents the central lens while $z_{k}$ is a lens at one of the vertices.

number of lenses previously studied in Section 5.1. In Table 5.3, allowed perturbations for the position are given in terms of the area of the Einstein disk for the asymmetric configuration, and in terms of the area of the critical disk for the central mass symmetric configuration. In the same table, allowed perturbations for the mass are given in terms of the mass of one of the lenses. ${ }^{9}$ From Table 5.3 it is then evident that the central mass symmetric configuration is more stable than the asymmetric configuration for $n=$ 5 point-mass lenses. Therefore, an asymmetric maximal lensing system with $n=5$ deflectors is less likely to be observed than the corresponding symmetric system with a small central mass. This behaviour is inverse to the one found for $n=4$ lenses (compare Tables 5.2 and 5.3).

It is worth noting that in the asymmetric configuration for $n=5$, the lens at $z_{1}$ can have its mass perturbed around a region that is $12.95 \%$ of the unperturbed value. This is much larger than the range allowed for the central mass symmetric configuration of $1.64 \%$ and is the only perturbation that showcases the asymmetric system as more stable than the central mass symmetric system. The most likely reason for the tolerance of the configuration under perturbations in the mass of the lens at $z_{1}$ is the location of this mass on an axis of symmetry. Therefore, larger changes in the mass are possible while leaving

\footnotetext{
${ }^{9}$ As for $n=4$, perturbations on the mass of the central lens are excluded here as those were studied in finding the upper bound $m_{\star}$ (see equation 3.9 ).
} 
the symmetry unbroken and thus maximal lensing is preserved. However, lenses at $z_{2}$ and $z_{3}$ are not located on an axis of symmetry and thus, perturbations on the masses of these lenses result in a breaking of the symmetry that leads to the loss of images.

In the simulations performed searching for an asymmetric maximal lensing configuration of $n=6$ equal point masses, the negative results allow us to set constraints on the stability of this configuration. At the maximum resolution of 0.002 in the position of the lenses, ${ }^{10}$ no maximal lensing configurations were found through the search schemes employed (see Sections 5.3 and B.3). Therefore, perturbations on a possible maximal lensing configuration that are larger than this value will result in the loss of images. That is, in the best case scenario, the area available for one of the lenses in the maximal lensing configuration has to be smaller than $0.0004 \%$ of the area of the Einstein disk. Given that the values obtained for the symmetric configuration of point-mass lenses in a pentagon with a small central mass indicate that perturbations in the position of one of the lenses can be as large as $0.0025 \%$ of the area of the critical disk, ${ }^{11}$ it is then clear that for $n=6$ lenses, asymmetric maximal lensing systems are less stable and thus less likely to be observed than the corresponding symmetric systems with a small central mass.

\footnotetext{
${ }^{10}$ Recall that distances on the lens plane are measured in units of the Einstein radius $R_{E}$.

${ }^{11}$ See Section 5.1 and in particular Figure 5.1.
} 


\section{Chapter 6}

\section{Conclusions}

Given the prominent place that gravitational lensing has found both as a tool in astrophysics and as a test for General Relativity, understanding the details of strong lensing and the production of multiple images is an important research goal.

We have found explicit bounds for the maximum number of images that a single-plane or thin lens configuration can produce in the case of deflectors described by point-mass lenses. These results are relevant as lenses modeled by point masses, or more precisely, distributions of the form of the Schwarzschild solution, provide good approximations to instances of lensing by stars and planets. Furthermore, from the study on the stability properties of the specific configurations that present maximal lensing we conclude that direct observation of the maximum number of images is unlikely except for the cases of small numbers of lenses. In addition, it is precisely through the analysis of the limited phase space available for maximal lensing in configurations with small numbers of lenses that we are able to use the mass bounds found as an application of gravitational lensing to the search for small mass companions in multiple star systems.

Extending point-mass lenses to mass profiles of finite non-zero radius, we have found the maximum number of images for configurations of distributed masses acting as lenses. The results of maximal lensing for extended mass distributions in a thin lens are suit- 
able to model instances found in cosmological scenarios where galaxies act as deflectors. As with point masses, the restrictions placed on maximal lensing configurations of distributed lenses can be used to set constraints that, in the cosmological case, lead to information on the presence of non-luminous matter and large-scale structure.

The main question that we defined as the guiding thread in the study of maximal lensing and image production is thus answered for the case of thin lenses; a set of $n$ point-mass lenses produces a maximum of $5(n-1)$ images of a distant luminous source, while a set of $n$ distributed mass lenses produces a maximum of $6(n-1)+1$ images of a distant luminous source.

\subsection{Future Research}

The next natural step in the study of maximal lensing is to extend our results to the case of thick lenses and multiplane gravitational lensing. The existing lower bound of $2^{n}$ images has been shown to be the minimum for $n$ point-mass lenses in $n$ separate planes. The upper bound of $2\left(2^{2(n-1)}-1\right)$ images for $n$ point-mass lenses at $n$ different distances to the observer was found through the theory of resultants and is the best result available. It is not know if this upper bound represents the maximum. A semi-analytic approach that combines complex analysis and the theory of resultants with a systematic set of simulations should lead to a better understanding of the multiplane lensing scenario and the refinement of the upper bound on the number of images. The extension of a multiplane result for point-mass lenses to the case of distributed lenses should follow a similar path to the one we used in the single-plane scenario. However, it is expected that in the multiplane scenario the particular plane where the lens is changed from point-mass to distributed mass will be a relevant factor in the calculation of the images added. This point adds complexity to the problem, in contrast to the single-plane configuration where the particular mass to be changed had no effect on the total number of images. 
Along a different direction, given that the upper bound found for the central mass perturbation that leads to maximal lensing in a symmetric configuration is not sharp, an extension of this work should focus on finding a better bound as a function of the radius of the regular polygon, or better yet, an analytic expression of the maximum mass. Not only would a result in this direction provide a more sound theoretical understanding of multiple imaging, but a better estimate of the boundaries can be used to refine maximal lensing configurations as a tool to search for exoplanets or brown dwarfs.

A study of the light curves for maximal lensing events when the system is perturbed will turn these results into an added tool for microlensing. The rationale behind this is that the determination of the particular signatures of a total magnification curve when the maximum number of images is present will be identifiable in those instances where multiple images present cannot be resolved. Furthermore, the sensitivity of maximal lensing to all perturbations, and in particular to the presence of small masses, will be reflected in light curves with signatures of these mass perturbations.

The small phase space found for maximal lensing in point-mass configurations as well as with distributed lenses (both in the case of symmetric configurations centrally perturbed and asymmetric configurations), highlights the low probability of direct observation. However, a statistical analysis of observed null cases can then be used to set stronger constraints on the distributions of lensing matter. 


\section{Bibliography}

Bayer, J. and Dyer, C. C. (2007). Maximal lensing: mass constraints on point lens configurations. General Relativity and Gravitation, 39:1413-1418.

Bayer, J., Dyer, C. C., and Giang, D. (2006). Gravitational lensing and the maximum number of images. General Relativity and Gravitation, 38:1379-1385.

Blandford, R. and Narayan, R. (1986). Fermat's principle, caustics, and the classification of gravitational lens images. ApJ, 310:568-582.

Boss, A. P. (2001). Formation of Planetary-Mass Objects by Protostellar Collapse and Fragmentation. ApJ, 551:L167-L170.

Bourassa, R. R. and Kantowski, R. (1975). The theory of transparent gravitational lenses. ApJ, 195:13-21.

Bourassa, R. R., Kantowski, R., and Norton, T. D. (1973). The Spheroidal Gravitational Lens. ApJ, 185:747-756.

Boyce, E. R., Winn, J. N., Hewitt, J. N., and Myers, S. T. (2005). Central Image Searches in Five Gravitational Lenses. In Bulletin of the American Astronomical Society, volume 37 of Bulletin of the American Astronomical Society, page 1498.

Boyce, E. R., Winn, J. N., Hewitt, J. N., and Myers, S. T. (2006). The Extragalactic Lens VLBI Imaging Survey (ELVIS). I. A Search for the Central Image in the Gravitational Lens PMN J1838-3427. ApJ, 648:73-80. 
Burke, W. L. (1981). Multiple Gravitational Imaging by Distributed Masses. ApJ, 244:L1.

Butkov, E. (1968). Mathematical physics. Addison-Wesley Series in Advanced Physics. Reading, Mass. etc.: Addison- Wesley Publishing Company. XI, 735 p.351.

Chwolson, O. (1924). Über eine mögliche Form fiktiver Doppelsterne. Astronomische Nachrichten, 221:329-330.

Clark, E. E. (1972). The uniform transparent gravitational lens. MNRAS, 158:233-243.

Cooke, J. H. and Kantowski, R. (1975). Time Delay for Multiply Imaged Quasars. ApJ, 195:L11-L14.

Dobler, G., Keeton, C. R., and Wambsganss, J. (2007). Microlensing of central images in strong gravitational lens systems. MNRAS, 377:977-986.

Dyer, C. C. (1973). Observational Aspects of Locally Inhomogeneous Cosmological Models. PhD thesis, AA(University of Toronto (Canada).).

Dyer, C. C. (1977). Optical scalars and the spherical gravitational lens. MNRAS, 180:231242.

Dyer, C. C. and Roeder, R. C. (1972). The Distance-Redshift Relation for Universes with no Intergalactic Medium. ApJ, 174:L115-L117.

Dyer, C. C. and Roeder, R. C. (1973). Distance-Redshift Relations for Universes with Some Intergalactic Medium. ApJ, 180:L31-L34.

Dyer, C. C. and Roeder, R. C. (1974). Observations in Locally Inhomogeneous Cosmological Models. ApJ, 189:167-176.

Dyer, C. C. and Roeder, R. C. (1980). Possible multiple imaging by spherical galaxies. ApJ, 238:L67-L70. 
Dyer, C. C. and Roeder, R. C. (1981a). On the transition from Weyl to Ricci focusing. General Relativity and Gravitation, 13:1157-1160.

Dyer, C. C. and Roeder, R. C. (1981b). Toward a realistic nebular gravitational lens. ApJ, 249:290-296.

Eddington, A. S. (1920). Space, time and gravitation. an outline of the general relativity theory. Cambridge Science Classics, Cambridge: University Press, 1920.

Einstein, A. (1915). Erklarung der Perihelionbewegung der Merkur aus der allgemeinen Relativitatstheorie. Sitzungsber. preuss.Akad. Wiss., vol. 47, No.2, pp. 831-839, 1915, $47: 831-839$.

Einstein, A. (1916). Die Grundlage der allgemeinen Relativitätstheorie. Annalen der Physik, 354:769-822.

Fraleigh, J. B. (1967). A first course in abstract algebra. Addison-Wesley Publishing Co., Reading, Mass.-London-Don Mills, Ont.

Friedmann, A. (1999). On the Curvature of Space. General Relativity and Gravitation, 31:1991-2000.

Gottlieb, D. H. (1994). A gravitational lens need not produce an odd number of images. Journal of Mathematical Physics, 35:5507-5510.

Guillemin, V. and Pollack, A. (1974). Differential topology. Prentice-Hall Inc., Englewood Cliffs, N.J.

Hu, E. M., Cowie, L. L., McMahon, R. G., Capak, P., Iwamuro, F., Kneib, J.-P., Maihara, T., and Motohara, K. (2002). A Redshift $\mathrm{z}=6.56$ Galaxy behind the Cluster Abell 370 . ApJ, 568:L75-L79. 
Inada, N., Oguri, M., Keeton, C. R., Eisenstein, D. J., Castander, F. J., Chiu, K., Hall, P. B., Hennawi, J. F., Johnston, D. E., Pindor, B., Richards, G. T., Rix, H.-W. R., Schneider, D. P., and Zheng, W. (2005). Discovery of a Fifth Image of the Large Separation Gravitationally Lensed Quasar SDSS J1004+4112. PASJ, 57:L7-L10.

Jenkins, M. A. and Traub, J. F. (1969/1970). A three-stage variable-shift iteration for polynomial zeros and its relation to generalized Rayleigh iteration. Numer. Math., $14: 252-263$.

Kayser, R. and Refsdal, S. (1983). The difference in light travel time between gravitational lens images. I - Generalization of the wavefront method to arbitrary deflectors and inhomogeneous universes. A\&A, 128:156-161.

Keeton, C. R., Mao, S., and Witt, H. J. (2000). Gravitational Lenses with more than Four Images. I. Classification of Caustics. ApJ, 537:697-707.

Khavinson, D. and Neumann, G. (2006). On the number of zeros of certain rational harmonic functions. Proc. Amer. Math. Soc., 134(4):1077-1085.

Kneib, J.-P., Ellis, R. S., Santos, M. R., and Richard, J. (2004). A Probable z 7 Galaxy Strongly Lensed by the Rich Cluster A2218: Exploring the Dark Ages. ApJ, 607:697703.

Lemaître, G. (1931). Expansion of the universe, A homogeneous universe of constant mass and increasing radius accounting for the radial velocity of extra-galactic nebulæ. MNRAS, 91:483-490.

Mao, S., Petters, A. O., and Witt, H. J. (1999). Properties of Point Mass Lenses on a Regular Polygon and the Problem of Maximum Number of Images. In Piran, T. and Ruffini, R., editors, Recent Developments in Theoretical and Experimental General Relativity, Gravitation, and Relativistic Field Theories, pages 1494-1496. 
McKenzie, R. H. (1985). A gravitational lens produces an odd number of images. Journal of Mathematical Physics, 26:1592-1596.

Milnor, J. (1963). Morse theory. Based on lecture notes by M. Spivak and R. Wells. Annals of Mathematics Studies, No. 51. Princeton University Press, Princeton, N.J.

Misner, C. W., Thorne, K. S., and Wheeler, J. A. (1973). Gravitation. San Francisco: W.H. Freeman and Co., 1973.

Narasimha, D., Subramanian, K., and Chitre, S. M. (1986). 'Missing image' in gravitational lens systems? Nature, 321:45-46.

O’Neill, B. (1983). Semi-Riemannian geometry, volume 103 of Pure and Applied Mathematics. Academic Press Inc. [Harcourt Brace Jovanovich Publishers], New York. With applications to relativity.

Padmanabhan, T. and Subramanian, K. (1988). The focusing equation, caustics and the condition for multiple imaging by thick gravitational lenses. MNRAS, 233:265-284.

Peacock, J. A. (1999). Cosmological Physics. Cosmological Physics, by John A. Peacock, pp. 704. ISBN 052141072X. Cambridge, UK: Cambridge University Press, January 1999.

Peebles, P. J. E. (1993). Principles of physical cosmology. Princeton Series in Physics, Princeton, NJ: Princeton University Press, |c1993.

Pelló, R., Schaerer, D., Richard, J., Le Borgne, J.-F., and Kneib, J.-P. (2004). ISAAC/VLT observations of a lensed galaxy at $z=10.0$. A\&A, 416:L35-L40.

Perlick, V. (1990). On Fermat's principle in general relativity. I. The general case. Classical and Quantum Gravity, 7:1319-1331.

Petters, A. O. (1992). Morse theory and gravitational microlensing. Journal of Mathematical Physics, 33:1915-1931. 
Petters, A. O. (1995a). Multiplane gravitational lensing. I. Morse theory and image counting. Journal of Mathematical Physics, 36:4263-4275.

Petters, A. O. (1995b). Multiplane gravitational lensing. II. Global geometry of caustics. Journal of Mathematical Physics, 36:4276-4295.

Petters, A. O. (1996). Lower Bounds on Image Magnification in Gravitational Lensing. Royal Society of London Proceedings Series A, 452:1475-1490.

Petters, A. O. (1997). Multiplane gravitational lensing. III. Upper bound on number of images. Journal of Mathematical Physics, 38:1605-1613.

Petters, A. O., Levine, H., and Wambsganss, J. (2001). Singularity theory and gravitational lensing. Singularity theory and gravitational lensing / Arlie O. Petters, Harold Levine, Joachim Wambsganss. Boston : Birkhäuser, c2001. (Progress in mathematical physics ; v. 21).

Renn, J., Sauer, T., and Stachel, J. (1997). The Origin of Gravitational Lensing: A Postscript to Einstein's 1936 Science Paper. Science, 275(5297):184-186.

Rhie, S. H. (1997). Infimum Microlensing Amplification of the Maximum Number of Images of n-Point Lens Systems. ApJ, 484:63-69.

Rhie, S. H. (2001). Can A Gravitational Quadruple Lens Produce 17 images? ArXiv Astrophysics e-prints. astro-ph/0103463.

Rhie, S. H. (2002). How Cumbersome is a Tenth Order Polynomial?: The Case of Gravitational Triple Lens Equation. ArXiv Astrophysics e-prints. astro-ph/0202294.

Rhie, S. H. (2003). n-point Gravitational Lenses with 5(n-1) Images. ArXiv Astrophysics e-prints. astro-ph/0305166.

Robertson, H. P. (1935). Kinematics and World-Structure. ApJ, 82:284-301. 
Rusin, D., Keeton, C. R., and Winn, J. N. (2005). Measuring Supermassive Black Holes in Distant Galaxies with Central Lensed Images. ApJ, 627:L93-L96.

Schneider, P. (1984). The amplification caused by gravitational bending of light. A\&A, 140:119-124.

Schneider, P. (1985). A new formulation of gravitational lens theory, time-delay, and Fermat's principle. A\&A, 143:413-420.

Schneider, P., Ehlers, J., and Falco, E. E. (1992). Gravitational Lenses. Gravitational Lenses, XIV, 560 pp. 112 figs.. Springer-Verlag Berlin Heidelberg New York. Also Astronomy and Astrophysics Library.

Schneider, P., Kochanek, C. S., and Wambsganss, J. (2006). Gravitational Lensing: Strong, Weak and Micro. In Meylan, G., Jetzer, P., and North, P., editors, Saas-Fee Advanced Course 33: Gravitational Lensing: Strong, Weak and Micro.

Shapiro, I. I. (1964). Fourth Test of General Relativity. Physical Review Letters, 13:789791.

Stephani, H. (1990). General Relativity, An Introduction to the Theory of Gravitational Field. General Relativity, An Introduction to the Theory of Gravitational Field, ISBN 0521370663, Cambridge University Press, 1990.

Subramanian, K. and Cowling, S. A. (1986). On local conditions for multiple imaging by bounded, smooth gravitational lenses. MNRAS, 219:333-346.

Uhlenbeck, K. (1975). A Morse theory for geodesics on a Lorentz manifold. Topology, 14:69-90.

Wald, R. M. (1984). General relativity. Chicago: University of Chicago Press, 1984.

Walker, A. G. (1935). On Riemannian spaces with spherical symmetry about a line and the conditions for isotropy in general relativity. $Q J$ Math, os-6(1):81-93. 
Walsh, D. (1989). 0957 + 561: The Unpublished Story. In Moran, J. M., Hewitt, J. N., and Lo, K.-Y., editors, Gravitational Lenses, volume 330 of Lecture Notes in Physics, Berlin Springer Verlag, pages 11-22.

Walsh, D., Carswell, R. F., and Weymann, R. J. (1979). $0957+561$ A, B - Twin quasistellar objects or gravitational lens. Nature, 279:381-384.

Wambsganss, J. (2000). Gravitational Lensing - A brief review. ArXiv Astrophysics e-prints. astro-ph/0012423.

Wambsganss, J. (2006). Gravitational lensing as a powerful astrophysical tool: Multiple quasars, giant arcs and extrasolar planets. Annalen der Physik, 15:43-59.

Weinberg, S. (1972). Gravitation and Cosmology: Principles and Applications of the General Theory of Relativity. Gravitation and Cosmology: Principles and Applications of the General Theory of Relativity, by Steven Weinberg, pp. 688. ISBN 0-471-925675. Wiley-VCH, July 1972.

Winn, J. N., Rusin, D., and Kochanek, C. S. (2004). The central image of a gravitationally lensed quasar. Nature, 427:613-615.

Witt, H. J. (1990). Investigation of high amplification events in light curves of gravitationally lensed quasars. A\&A, 236:311-322.

Witt, H. J. and Petters, A. O. (1993). Singularities of the one- and two-point mass gravitational lens. Journal of Mathematical Physics, 34:4093-4111. 


\section{Appendix A}

\section{Some Mathematical Proofs}

In this appendix we present the mathematical proofs of stated properties for the critical radius $r_{c r}$ (see equation 3.1) and the upper bound mass $m_{\star}$ (see equation 3.9) found in Chapter 3 .

\section{A.1 Minimum Value for the Critical Radius}

In Section 3.1 we showed that a maximal lensing configuration was obtained by perturbing an array of $n-1$ identical point-mass lenses located at the vertices of a regular polygon of $n-1$ sides with a small mass at the center of the configuration. A necessary condition for maximal lensing is that the radius of the polygon must be smaller than a critical value $r_{c r}$ given in (3.1). Here we prove that the critical radius, as a function of the number of vertices in the polygon $n-1$, has a minimum at $r_{c r}=\frac{1}{\sqrt{2}}$ for $n-1=4$ and is bounded above so that $r_{c r}<1$.

Consider the function $f(x)$ given by

$$
f(x)=\left(\left[\frac{x-2}{x}\right]^{(x-2) / 2}-\left[\frac{x-2}{x}\right]^{x / 2}\right)^{1 / x}
$$

so that for $n \in \mathbb{N}$ and $n \geq 4$, the critical radius is $r_{c r}=f(n-1)$. The derivative of $f(x)$ 
is given by

$$
f^{\prime}(x)=\frac{2^{\frac{1-x}{x}}\left(x^{-\frac{x}{2}}(x-2)^{\frac{x-2}{2}}\right)^{\frac{1}{x}}\left(x \ln (x-2)-x \ln x-2 \ln \left(2 x^{-\frac{x}{2}}(x-2)^{\frac{x-2}{2}}\right)\right)}{x^{2}} .
$$

In order to find the critical points, given that $n \geq 4$ the domain of definition for $f(x)$ can be restricted to $x \in[3, \infty)$. Therefore $f^{\prime}\left(x_{0}\right)=0$ if and only if

$$
g\left(x_{0}\right) \equiv x_{0} \ln \left(x_{0}-2\right)-x_{0} \ln x_{0}-2 \ln \left(2 x_{0}^{-\frac{x_{0}}{2}}\left(x_{0}-2\right)^{\frac{x_{0}-2}{2}}\right)=0 .
$$

Solutions to equation (A.2) can then be found in a simpler way by solving instead

$$
e^{g\left(x_{0}\right)}=1
$$

as using the explicit form of equation (A.2), we can write (A.3) as

$$
\frac{\left(x_{0}-2\right)^{2}}{4}=1
$$

The solution $x_{0}=0$ for equation (A.4) must be discarded as it lies outside the restricted domain for $f(x)$. Thus, the remaining solution of (A.4) gives $x_{0}=4$ as a critical point of $f(x)$.

The second derivative of $f(x)$ is given by

$$
\begin{aligned}
f^{\prime \prime}(x) & =2^{\frac{1-2 x}{x}} x^{-4}(x-2)^{-1}\left(x^{-\frac{x}{2}}(x-2)^{\frac{x-2}{2}}\right)^{\frac{1}{x}} \\
& \times\left(4 x^{2}+(x-2)\left(x \ln (x-2)-x \ln x-2 \ln \left(2 x^{-\frac{x}{2}}(x-2)^{\frac{x-2}{2}}\right)\right)\right) \\
& \times\left(x \ln (x-2)-x(\ln x+4)-2 \ln \left(2 x^{-\frac{x}{2}}(x-2)^{\frac{x-2}{2}}\right)\right),
\end{aligned}
$$

and therefore at $x_{0}=4$ we obtain

$$
f^{\prime \prime}\left(x_{0}\right)=\frac{1}{32 \sqrt{2}}
$$


Since for $x_{0}=4$ we have $f^{\prime}\left(x_{0}\right)=0$ and $f^{\prime \prime}\left(x_{0}\right)>0$ then $f\left(x_{0}\right)=\frac{1}{\sqrt{2}}$ is a minimum. Given that at $x_{1}=3$ we have

$$
f\left(x_{1}\right)=\frac{2^{\frac{1}{3}}}{\sqrt{3}}>\frac{1}{\sqrt{2}},
$$

then $\frac{1}{\sqrt{2}}$ is an absolute minimum for $f(x)$ when $x \in[3, \infty)$. Given that $r_{c r}=f(n-1)$ it follows that $r_{c r} \geq \frac{1}{\sqrt{2}}$ and the minimum occurs at $n-1=4$.

Since the only critical point of $f(x)$ in the interval $[3, \infty)$ is $x_{0}=4$, the maximum value is located either at $x_{1}=3$ or at the limit when $x \rightarrow \infty$. To calculate the limit, first note that $f(x)$ from equation (A.1) can be simplified to

$$
f(x)=2^{\frac{1}{x}} x^{-\frac{1}{2}}(x-2)^{\frac{x-2}{2 x}},
$$

and taking the natural logarithm

$$
\ln f(x)=\frac{\ln 2}{x}-\frac{\ln x}{2}+\frac{x-2}{2 x} \ln (x-2)=\frac{\ln 2}{x}+\frac{1}{2} \ln \left(\frac{x-2}{x}\right)-\frac{\ln (x-2)}{x} .
$$

Taking the limit as $x \rightarrow \infty$ in equation (A.6) we obtain

$$
\lim _{x \rightarrow \infty} \ln f(x)=0,
$$

so that

$$
\lim _{x \rightarrow \infty} f(x)=1
$$

Given that $f\left(x_{1}=3\right)<1$ (see equation A.5) and the limit in (A.7) it follows that for all $x \in[3, \infty)$ we have $f(x)<1$ so that $r_{c r}<1$.

To summarize, in this section we have proved that the critical radius $r_{c r}$ for maximal lensing of $n-1$ point-mass lenses located at the vertices of a regular polygon of $n-1$ 
sides $^{1}$ is bounded according to

$$
\frac{1}{\sqrt{2}} \leq r_{c r}<1
$$

and the minimum is obtained in the case of $n-1=4$ lenses.

\section{A.2 Monotonicity of the Bound for the Central Mass}

In Section 3.1 we showed that an upper bound for the central mass that can be placed in a symmetric configuration in order to produce the maximum number of images was given by $m_{\star}$ in equation (3.9). We studied the properties of this upper bound in Section 3.2 and in particular we mentioned that $m_{\star}$ increases with the radius $r$ of the polygon of point masses. Here we present the proof that for $r<1$ and $n \geq 4$ the derivative $\frac{\partial m_{\star}}{\partial r}$ given in equation (3.10) is positive, completing the proof that $m_{\star}$ increases with $r$.

Recall from equation (3.10) that $\frac{\partial m_{\star}}{\partial r}$ is given by

$$
\frac{\partial m_{\star}}{\partial r}=\frac{2\left((n+5) r^{\frac{n+11}{n-1}}\left(1+r^{6}\right)^{2}-3 r^{5}(n-1)\right)}{(n-1)\left(1+r^{6}\right)^{2}}
$$

therefore, $\frac{\partial m_{\star}}{\partial r}>0$ if and only if $f(r, n)>0$ for

$$
f(r, n) \equiv(n+5) r^{\frac{n+11}{n-1}}\left(1+r^{6}\right)^{2}-3 r^{5}(n-1) .
$$

In order to show that $f(r, n)>0$, we proceed by mathematical induction on the number of point-mass lenses $n$. The base case for $n=4$ gives

$$
f(r, 4)=9 r^{11}\left(2+r^{6}\right)
$$

so that given $r>0$ we have $f(r, 4)>0$. For the inductive step we must prove that

\footnotetext{
${ }^{1}$ Note that since $n-1$ is the number of sides of the polygon it follows that $n-1 \geq 3$.
} 
if $f(r, k)>0$ then $f(r, k+1)>0$ and thus mathematical induction will imply that $f(r, n)>0$ for all $n \geq 4$. The condition $f(r, k)>0$ is

$$
f(r, k)=(k+5) r^{\frac{k+11}{k-1}}\left(1+r^{6}\right)^{2}-3 r^{5}(k-1)>0
$$

and we must use this to prove

$$
f(r, k+1)=(k+6) r^{\frac{k+12}{k}}\left(1+r^{6}\right)^{2}-3 r^{5} k>0 .
$$

Reorganizing the terms of $f(r, k+1)$ we have

$$
\begin{aligned}
f(r, k+1)= & (k+5) r^{\frac{k+12}{k}}\left(1+r^{6}\right)^{2}-3 r^{5}(k-1)+r^{\frac{k+12}{k}}\left(1+r^{6}\right)^{2}-3 r^{5} \\
= & (k+5) r^{\frac{k+11}{k-1}}\left(1+r^{6}\right)^{2}-3 r^{5}(k-1) \\
& +r^{\frac{k+12}{k}}\left(1+r^{6}\right)^{2}-3 r^{5}+(k+5) r^{\frac{k+11}{k-1}}\left(r^{-\frac{12}{k(k-1)}}-1\right)\left(1+r^{6}\right)^{2} \\
= & f(r, k)+g(r, k),
\end{aligned}
$$

where we have defined

$$
g(r, k) \equiv r^{\frac{k+12}{k}}\left(1+r^{6}\right)^{2}-3 r^{5}+(k+5) r^{\frac{k+11}{k-1}}\left(r^{-\frac{12}{k(k-1)}}-1\right)\left(1+r^{6}\right)^{2}
$$

Given the inductive hypothesis (A.8) and equation (A.10), to show that $f(r, k+1)>0$, it is sufficient to prove $g(r, k)>0$. Reorganizing terms in (A.11) we have

$$
\begin{aligned}
g(r, k) & =r\left(r^{\frac{12}{k}}\left(1+r^{6}\right)^{2}+(k+5) r^{\frac{12}{k-1}}\left(r^{-\frac{12}{k(k-1)}}-1\right)\left(1+r^{6}\right)^{2}-3 r^{4}\right) \\
& =r\left(r^{\frac{12}{k}}\left(1+r^{6}\right)^{2}\left[1+(k+5) r^{\frac{k}{k-1}}\left(r^{-\frac{12}{k(k-1)}}-1\right)\right]-3 r^{4}\right) \\
& =r\left(r^{\frac{12}{k}}\left(1+r^{6}\right)^{2}\left[1+(k+5)\left(r^{-\frac{12}{(k-1)^{2}}}-r^{\frac{k}{k-1}}\right)\right]-3 r^{4}\right) .
\end{aligned}
$$

Since $r<1$ and $k>4$, it follows that

$$
r^{-\frac{12}{(k-1)^{2}}}>1 \text { and } r^{\frac{k}{k-1}}<r
$$


so that

$$
\left(r^{-\frac{12}{(k-1)^{2}}}-r^{\frac{k}{k-1}}\right)>(1-r)
$$

Replacing (A.13) into the last line of (A.12) we obtain

$$
\begin{aligned}
g(r, k) & >r\left(r^{\frac{12}{k}}\left(1+r^{6}\right)^{2}[1+(k+5)(1-r)]-3 r^{4}\right) \\
& >r\left(r^{3}\left(1+r^{6}\right)^{2}[1+9(1-r)]-3 r^{4}\right) \\
& >r^{4}\left(\left(1+r^{6}\right)^{2}+9\left(1+r^{6}\right)^{2}(1-r)-3 r\right)
\end{aligned}
$$

where we have used again the fact that $r<1$ and $k>4$ so that $r^{\frac{12}{k}}>r^{3}$ and $(k+5)>9$. Given that $r>0$ it follows that

$$
\left(1+r^{6}\right)^{2}>1+2 r^{6}>1
$$

and using (A.15) in the last line of (A.14) we arrive at

$$
\begin{aligned}
g(r, k) & >r^{4}\left(\left(1+2 r^{6}\right)+9(1-r)-3 r\right) \\
& >2 r^{4}\left(r^{6}-6 r+5\right) \\
& >2 r^{4} h(r)
\end{aligned}
$$

where

$$
h(r) \equiv r^{6}-6 r+5 .
$$

Given that $h^{\prime}(r)=6\left(r^{5}-1\right)$, the only critical point of $h(r)$ is $r_{0}=1$. Furthermore, since $h^{\prime \prime}(r)=6 r^{4}$ so that $h^{\prime \prime}\left(r=r_{0}\right)=6>0$, then $h(r)$ has an absolute minimum at $r_{0}=1$ given by $h\left(r_{0}\right)=0$. Since in the case under study $r<1$ we then have $h(r)>0$ and using (A.16) into (A.10) along with the induction hypothesis (A.8) it follows that

$$
f(r, k+1)>0 .
$$


Therefore, the principle of mathematical induction guarantees that

$$
\frac{\partial m_{\star}}{\partial r}=\frac{2 f(r, n)}{(n-1)\left(1+r^{6}\right)^{2}}>0
$$

for $0<r<1$ and $n \geq 4$, completing the proof. 


\section{Appendix B}

\section{Numerical Simulations}

In this appendix we present the Mathematica codes used in the numerical simulations throughout the research. These numerical simulations were used in the semi-analytical approach that led to the upper-bound mass for the maximal lensing configuration described in Sections 3.1 and 3.2. In addition, the numerical simulations were employed to analyze the stability properties of maximal lensing configurations in Sections 5.1, 5.2 and 5.4, as well as to find the asymmetric maximal lensing configurations in Section 5.3.

The basic algorithm for all the codes used can be described in the following steps:

1. Set Parameters: In this part the parameters of the lensing configuration under study are defined. If the code is meant to search for the value of a specific parameter that leads to a desired image production, the parameter and its phase space are defined here. For example if the goal is to find the mass in one lens that leads to maximal lensing, that mass is defined as a function of a variation parameter.

2. Witt's Polynomial: In this part, the code generates the polynomial that represents an analytic embedding of the lens equation in terms of the lens parameters defined. See Section 2.3, in particular the derivation of equation (2.18) for details on the polynomial representation. This polynomial is then a function of the variation parameter previously determined. In the case described in the previous step, then 
the polynomial will be a function of the parameter that describes the phase space of possible masses.

3. Search Parameter Space: For a defined range of the chosen variation parameter a search is performed until an exit condition is met. In the case highlighted as an example, the values of the mass are iterated at a set resolution in the range defined until the number of images is maximal. The search proceeds carrying the following steps for each iteration:

(a) Solutions to the Polynomial: Using the algebraic and numerical polynomial solving algorithms in Mathematica, the solutions for Witt's polynomial are found. ${ }^{1}$

(b) Solutions to the Lens Equation: The solutions of the polynomial are tested in the lens equation to separate real images from those solutions that are a product of the analytic embedding.

(c) Lensing Plots: Diagrams or plots with information on critical curves, caustics, real images, lens positions and magnifications are produced when helpful to illustrate the situation.

(d) Exit Condition: Test for the exit condition in the search. In the case used as an example, the exit condition will be that the the system produces the maximum number of images for the number of lenses defined.

\section{B.1 Mass Finding Code}

The code in this section was used to search for the maximum value of the central mass perturbation that presented maximal lensing when placed in a symmetric lens configura-

\footnotetext{
${ }^{1}$ Mathematica uses the Jenkins-Traub algorithm (Jenkins and Traub, 1970) to find the zeros of polynomials.
} 
tion as described in Section 3.2. The search was run for various values of the radius of the polygon and for increasing values of the number of lenses in the polygon. Furthermore, the resolution in the search was refined after each run to reduce the error bars in the mass found. Computational limitations set the practical limit for these simulations at $n=100$ lenses because past this point the problem of generating Witt's polynomial of degree $\operatorname{deg} p=1001$ using equation (2.18) exceeds the memory resources available.

The sample code listed below is for the case of $n-1=10$ point-mass lenses at the vertices of a regular decagon of radius $r=\frac{1}{2}$.

(* Load package for graphing multiple functions at a time *)

$<<$ Graphics'MultipleListPlot'

(* This defines the value for $\mathrm{n}$ masses in the lens configuration $*$ )

ene $=11$;

(* Critical radius for $\mathrm{n}-1$ lenses in the polygon *)

$\operatorname{rhom}\left[n_{-}\right]:=\operatorname{Sqrt}[(\mathrm{n}-3) /(\mathrm{n}-1)]$;

rcritical $\left[n_{-}\right]:=\left(\operatorname{rhom}[n]^{\wedge}(n-3)-\operatorname{rhom}[n]^{\wedge}(n-1)\right)^{\wedge}(1 /(n-1))$;

(* The $\mathrm{n}-1$ masses located along the lines of the $(\mathrm{n}-1)$ - sided polygon *)

$\mathrm{a}\left[\mathrm{i}_{-}\right]:=\left\{\operatorname{Cos}\left[2 * \mathrm{Pi}^{*}(\mathrm{i}-1) /(\right.\right.$ ene -1$\left.)\right], \operatorname{Sin}\left[2 * \mathrm{Pi}^{*}(\mathrm{i}-1) /(\right.$ ene -1$\left.\left.)\right]\right\}$;

(* The central mass perturbation ... at the center $*$ )

a[ene] $:=\{0,0\}$;

(* Positions of the masses at the vertices of the polygon and radius of the polygon *)

$z\left[i_{-}, s_{-}\right]:=a[i] / 2$;

(* The central mass perturbation ... at the center *)

z[ene, s_] :=a[ene];

(* The $\mathrm{n}-1$ masses with a normalized total of $1^{*}$ )

ml[i_,s_] := 1/(ene - 1$)$;

(* The central mass perturbation with a fraction of the total mass to search for *)

$\mathrm{ml}[$ ene, s_] :=s;

(* Position of the source *)

zs : $=\{0,0\}$;

(* Array with the location of all the lenses *)

lentes[s_] := Table[z[i, s], $\{i$, ene $\}]$;

(* First section of Witt's polynomial *)

na $=$ Expand[Table $[($ aux $=1$; For $[k=1, k<=$ ene, $k++$, If $[k !=j$, 
aux $=\operatorname{aux}(z[k, r][[1]]+I z[k, r][[2]]-w)]] ;$ aux $),\{j, 1$, ene +1$\}]] ;$

(* Second section of Witt's polynomial *)

$\mathrm{nb}=\operatorname{Expand}\left[\operatorname{Sum}\left[\mathrm{ml}[\mathrm{i}, \mathrm{r}]^{*}\right.\right.$ na[[i]], $\{\mathrm{i}, 1$, ene $\left.\left.\}\right]\right] ;$

(* Third section of Witt's polynomial *)

$\mathrm{nc}=\operatorname{Expand}[(\mathrm{w}-(\mathrm{zs}[[1]]+\mathrm{I} \mathrm{zs}[[2]])) *($ aux $=1 ;$ For $[j=1, \mathrm{j}<=$ ene, $\mathrm{j}++$, aux $=$ aux* $(((z[j, r][[1]]-I z[j, r][[2]])-(z s[[1]]-\mid z s[[2]]))$

$*_{\text {na }}[[$ ene +1$\left.\left.]]+\mathrm{nb}\right)\right]$; aux $\left.)\right]$;

(* Fourth section of Witt's polynomial *)

nd $=$ Expand[Sum[m|[i, r]*(aux $=1$; For $[j=1, j<=$ ene, $j++$, If $[j !=i$, aux $=$ aux* $(((z[j, r][[1]]-\mathrm{I} z[j, \mathrm{r}][[2]])-(z \mathrm{~s}[[1]]-\mathrm{I} z \mathrm{~s}[[2]]))$

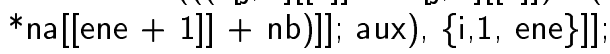

(* Fifth section of Witt's polynomial *)

ne $=$ Expand[na[[ene +1$]]^{*}$ nd];

(* Free up some memory *)

ClearAll[na, nb, nd]; Share[];

(* Witt's Polynomial *)

poly[z_, s_] $:=$ Expand $[\mathrm{nc}+\mathrm{ne}] / .\{\mathrm{w}->\mathrm{z}, \mathrm{r}->\mathrm{s}\}$;

(* Real and imaginary parts of the lens equation to test Witt's solutions *) equis[x_, y_, s_] := x - Sum[m|[k, s] $(x-z[k, s][[1]]) /((x-z[k, s][[1]]) \wedge 2$ $+(\bar{y}-z[k, \bar{s}][[2]]) \wedge 2),\{k$, ene $\}] ;$

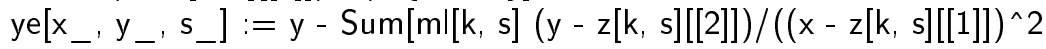

$\left.+(y-z[k, s][[2]])^{\wedge} 2\right),\{k$, ene $\left.\}\right]$

(* Magnification function for images *)

$\operatorname{mag}\left[i m g \_, s_{-}\right]:=1 /\left(1-\left(\operatorname{Abs}\left[\operatorname{Sum}\left[\mathrm{ml}[\mathrm{k}, \mathrm{s}] /\left(\left(\mathrm{img}[[1]]+\mathrm{I}{ }{ }_{i m g}[[2]]\right)\right.\right.\right.\right.\right.$

$\left.-\left(z[k, s][[1]]+I^{*} z[k, s][[2]]\right)\right)^{\wedge} 2,\{k, 1$, ene $\left.\left.\left.\left.\}\right]\right]\right)^{\wedge} 2\right)$;

(* Parity function for images *)

par[img_, s_] := Sign[mag[img, s]];

(* Equation for the critical curves *)

$\mathrm{p}\left[\mathrm{z}_{-}\right.$, theta_, $\left.\mathrm{s}_{-}\right]:=\operatorname{Product}\left[(\mathrm{z}-(\mathrm{z}[\mathrm{j}, \mathrm{s}][[1]]+\mathrm{I}[\mathrm{zj}, \mathrm{s}][[2]]))^{\wedge} 2,\{\mathrm{j}\right.$, ene $\left.\}\right]$

- Exp[I theta] (aux =0; For $[k=1, k<=$ ene, $k++$, otto $=1$;

For $[j=1, j<=$ ene, $j++$, If $[j !=k$, otto $=$ otto

$\left.\left.*(z-(z[j, s][[1]]+\mid z[j, s][[2]]))^{\wedge} 2\right]\right] ;$ aux = aux + ml[k, s] otto]; aux $)$;

$(*$ Main loop *)

(* Clear all variables and initialize parameters *)

ClearAll[graph, centerplus, centerminus, aux, witt, critical, zap, amp,

mmax, mmin, caustic, images, ampmax, sspace, scaledamp, scap, slow];

graph $=\{\}$

amp $=\{\}$

ampmax $=0$;

images $=\{\}$

$\operatorname{mmax}=0$;

$\operatorname{mmin}=5$ ene -5 ; 


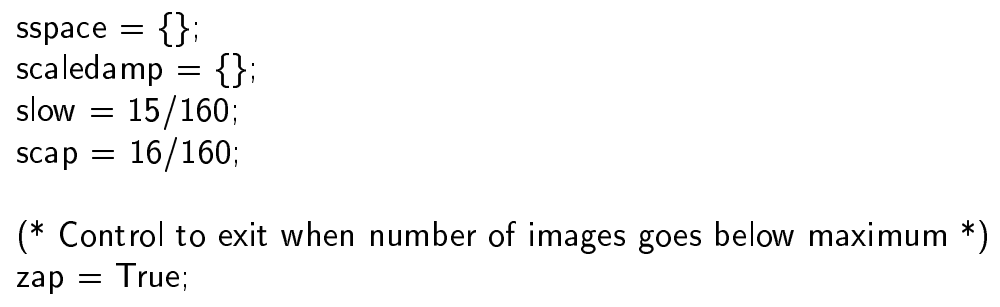

(* Search loop for s between slow and scap *)

For $[s=$ slow, $s<=$ scap \&\& zap, $s+=1 / 3200$,

$(*$ Numerical expression for Witt's polynomial $*$ )

aux $=\mathrm{N}[$ poly[w, s], 200];

(* Find all the solutions of the polynomial *)

witt $=$ Solve[aux $==0, \mathrm{w}]$;

(* Reorganize the solutions from complex numbers to ordered pairs *)

aux $=$ Table[w /. Extract[witt, i], $\{i, 1$, Length[witt] $\}]$;

witt $=$ Table[\{Re[aux[[i]]], Im[aux[[i]]]\}, \{i, 1, Length[aux]\}];

(* Auxiliary variable to sort the solutions *)

centerplus $=$ witt;

(* Remove solutions at the position of a lensing mass that generate divergences *)

For[ $k=1, k<=$ ene, $k++$, For[j $=1, \mathrm{j}<=$ Length[centerplus], $\mathrm{j}++$,

If $[(($ centerplus[[j]]][1]] $-z[k, s][[1]]) \wedge 2+($ centerplus[[j]]][2]]]

$\left.-z[k, s][[2]])^{\wedge} 2\right)==0$, centerplus $=$ Drop[centerplus, $\left.\left.\left.\left.\{j, j\}\right] ; j-\right]\right]\right] ;$

(* Remove duplicate solutions *)

For[ $j=1, j<=$ Length[centerplus], $\mathrm{j}++$, For[ $k=\mathrm{j}+1, \mathrm{k}<=$ Length[centerplus],

$\mathrm{k}++$, If[centerplus[[j]] $==$ centerplus[[k]], centerplus $=$

Drop[centerplus, $\{k, k\}] ; \mathrm{k}-]]$;

(* Remove solutions that do not satisfy the lens equation *)

For[j $=1, j<=$ Length[centerplus], $\mathrm{j}++$, If[Chop[equis[centerplus[[j]][[1]],

centerplus[[j]][[2]], s] - zs[[1]], 10^-20] !=0 || Chop[ye[centerplus[[j]][[1]],

centerplus[[j]][[2]], s] - zs[[2]], 10^-20] $!=0$, centerplus $=$

Drop[centerplus, $\{\mathrm{j}, \mathrm{j}\}]$; j-]];

(* Make a list of the number of images as a function of $s *$ )

images $=$ Append[images, $\{\mathrm{s}$, Length[centerplus]\}];

(* Exit condition if producing less than the maximum number of images *)

$(*$ When not searching for maximum mass, remove and make light curves $*$ )

zap $=$ zap \&\& (Length[centerplus] $==5$ ene -5$)$;

(* Test for maximum and minimum number of images found *)

If[Length[centerplus] $>$ mmax, $\operatorname{mmax}=$ Length[centerplus]];

If[Length[centerplus] $<$ mmin, $\operatorname{mmin}=$ Length[centerplus]];

(* Test for the largest amplification factor *)

If[Sum[Abs[mag[centerplus[[i]], s]], \{i, Length[centerplus]\}] > ampmax,

ampmax $=\mathrm{N}[$ Round[Sum[Abs[mag[centerplus[[i]], s]], $\{\mathrm{i}$, Length[centerplus]\}]]];

$(*$ Calculate the total amplification for all the images present $*$ )

amp = Append[amp, $\{\mathrm{s}, \operatorname{Sum}[\mathrm{Abs}[\mathrm{mag}[$ centerplus[[i]], $\mathrm{s}]],\{\mathrm{i}$, Length[centerplus]\}]\}];

centerminus $=\{\}$;

(* Separate images of positive and negative magnification *)

For[j $=1, j<=$ Length[centerplus], $j++$, If[par[centerplus[[j]], s] == -1 ,

centerminus $=$ Append [centerminus, centerplus[[j]]]; centerplus $=$

Drop[centerplus, $\{j, j\}] ; j-]]$;

aux $=\{\}$;

critical $=\{\}$;

( Calculate the critical curves $*$ )

For[phi $=0$, phi $<2 \mathrm{Pi}$, phi $+=2 \mathrm{Pi} / 10$, aux $=\operatorname{Solve}[\mathrm{N}[\mathrm{p}[\mathrm{z}, \mathrm{phi}, \mathrm{s}], 10]==0, \mathrm{z}]$; 
For $[\mathrm{j}=1, \mathrm{j}<=$ Length[aux], $\mathrm{j}++$, critical = Append[critical,

$\{\operatorname{Re}[z] /$. aux[[j]], $\operatorname{Im}[z] /$. aux [[j]]\}]]];

caustic $=\{\}$;

(* Calculate the caustics *)

For[j $=1, \mathrm{j}<=$ Length[critical], $\mathrm{j}++$, caustic $=$ Append[caustic,

\{equis[critical[[j]][[1]], critical[[j]][[2]], s], ye[critical[[j]][[1]],

critical[[j]][[2]], s]\}]];

(* Graph images, lenses, source, witt's images and caustic and critical curves *)

graph $=$ Append[graph, MultipleListPlot[centerplus, centerminus, lentes[s], $\{z s\}$,

critical, witt, caustic, SymbolShape $->$ \{PlotSymbol[Star, 5, Filled $->$ False],

PlotSymbol[Star, 5, Filled -> False], PlotSymbol[Box, 3, Filled -> False],

PlotSymbol[Diamond, 3], PlotSymbol[Box, 0.5], PlotSymbol[Diamond, 2],

PlotSymbol[Box, 0.5]\}, SymbolStyle $->\{$ RGBColor[0, 0, 1], RGBColor[1, 0, 0],

RGBColor[0, 0.5, 0.5], RGBColor[1, 0, 1], RGBColor[1, 0, 0.5], RGBColor[0, 0, 0],

RGBColor[0, 0.5, 0.5]\}, AspectRatio -> Automatic, ImageSize $->85 * 10$,

PlotRange $->\{\{-1.5,1.5\},\{-1.5,1.5\}\}]]$;

( Clear parameters for next iteration *)

ClearAll[aux, centerplus, centerminus, witt, critical, caustic]];

(* Return max and min number of images and max amplification *)

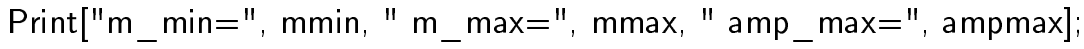

scaledamp $=\{\}$

(* Scale the magnification to plot alongside the number of images *)

scaledamp $=$ Table $[\{\operatorname{amp}[[i]][[1]]$, amp $[[i]][[2]] *(5$ ene -5$) /$ ampmax $+($ ene +1$) / 2\}$,

$\{i, 1$, Length[amp]\}];

(* Graph magnification and number of images as a function of $s *$ )

(* Graph a light curve with maximal lensing signature *)

MultipleListPlot[images, scaledamp, Plot Joined $->\{$ True, True $\}$,

PlotStyle $->\{$ RGBColor $[1,0,0], \operatorname{RGBColor}[0,0,1]\}$, ImageSize $->85^{*} 10$,

PlotRange $->\{\{$ slow, scap $\},\{0$, Round $[(5$ ene -5$)+($ ene +1$) / 2]\}\}$,

SymbolShape $->\{$ PlotSymbol[Box, 1], PlotSymbol[Box, 1]\},

SymbolStyle $->\{$ RGBColor[1, 0, 0], RGBColor[0, 0, 1]\}];

sspace $=\{\} ;$

(* Extract values for the central mass that given maximal lensing *)

For $[i=1, i<=$ Length[images], i++, If[images[[i]][[2]] $==(5$ ene -5$)$,

sspace $=$ Append[sspace, images[[i]][[1]]]]];

(* Return the values of mass for maximal lensing *)

Print[sspace];

\section{B.2 Perturbations on Symmetric Configurations}

To test the stability of the maximal lensing configuration of $n-1$ point-masses at the vertices of a regular polygon of $n-1$ sides plus a small mass at the center of the polygon, numerical simulations were run perturbing the position of source, central mass and a lens on the polygon as well as the mass of a lens on the polygon. The results of these simulations are presented in Section 5.1 and the code used is presented here. The limiting 
resource for the simulations is the memory available to generate Witt's polynomial (2.18) and in this case due to the breaking of symmetries introduced by the perturbations, the largest value of the number of lenses tested was $n=14$. For each value of $n$, the radius of the polygon was set at $r=\frac{r_{c r}}{2}$, where $r_{c r}$ is given by equation (3.1). Furthermore, the value of the central mass perturbation was chosen as $m=\frac{m_{\max }}{2}$, where $m_{\max }$ is the maximum central mass for maximal lensing for the polygon of radius $r=\frac{r_{c r}}{2}$, found through the numerical simulations detailed in Section B.1.

The sample code listed below is for the case of $n-1=6$ point-mass lenses at the vertices of a regular hexagon with a central mass $m=\frac{93}{10000}$. The angular position of one of the masses in the polygon is perturbed in order to find the region of stability that presents maximal lensing. Important differences between this code and the one listed in Section B.1 are highlighted in bold typeface.

(* Load package for graphing multiple functions at a time *)

$<<$ Graphics'MultipleListPlot'

$(*$ This defines the value for $\mathrm{n}$ masses in the lens configuration $*$ )

ene $=7$;

(* Critical radius for $\mathrm{n}-1$ lenses in the polygon *)

$\operatorname{rhom}\left[\mathrm{n}_{-}\right]:=\operatorname{Sqrt}[(\mathrm{n}-3) /(\mathrm{n}-1)]$;

$\operatorname{rcritical}\left[n_{-}\right]:=\left(\operatorname{rhom}[n]^{\wedge}(n-3)-\operatorname{rhom}[n]^{\wedge}(n-1)\right)^{\wedge}(1 /(n-1))$;

(* The $\mathrm{n}-1$ masses located along the lines of the $(\mathrm{n}-1)$ - sided polygon *)

$a\left[i_{-}, s_{-}\right]:=\{\operatorname{Cos}[2 * P i *(i-1) /($ ene -1$)], \operatorname{Sin}[2 * P i *(i-1) /($ ene -1$)]\}$;

$(* \bar{T}$ The central mass perturbation ... at the center $*$ )

a[ene,s_] $:=\{0,0\}$;

(* Perturbed tangential position of the first lens *)

$a\left[1, s_{-}\right]:=\left\{\operatorname{Cos}\left[2 * \operatorname{Pi}^{*}(1-1) /(\right.\right.$ ene -1$\left.)+s / 100\right], \operatorname{Sin}\left[2 * P_{i} *(1-1) /(\right.$ ene -1$\left.\left.)+s / 100\right]\right\}$

(* Positions of the masses at the vertices of the polygon and radius of the polygon *) $z\left[i_{-}, s_{-}\right]:=\operatorname{rcritical}[e n e] * a[i, s] / 2$;

(* The central mass perturbation ... at the center $*$ )

z[ene, s_] := rcritical[ene]*a[ene,s];

(* The $\overline{\mathrm{n}}-1$ masses with a normalized total of $1^{*}$ )

$\mathrm{ml}\left[i_{-}, \mathrm{s}_{-}\right]:=1 /($ ene -1$)$;

(* The central mass equal to half the upper bound for maxmimal lensing *)

ml[ene, s_] := 93/10000;

(* Position of the source *)

zs $:=\{0,0\}$; 
(* Array with the location of all the lenses *)

lentes[s_] := Table[z[i, s], $\{i$, ene $\}]$;

(* First section of Witt's polynomial *)

na $=$ Expand[Table $[($ aux $=1$; For $[k=1, k<=$ ene, $k++$, If $[k !=j$,

aux = aux*(z[k, r][[1]] + I z[k, r][[2]] - w) $]] ;$ aux $),\{j, 1$, ene +1$\}]]$;

(* Second section of Witt's polynomial *)

$\mathrm{nb}=\operatorname{Expand}\left[\right.$ Sum[m|[i, r] ${ }^{*}$ na[[i]], $\{i, 1$, ene $\left.\left.\}\right]\right] ;$

(* Third section of Witt's polynomial *)

$n c=\operatorname{Expand}[(w-(z s[[1]]+\mid \mathrm{zs}[[2]])) *(\operatorname{aux}=1 ; \operatorname{For}[j=1, \mathrm{j}<=$ ene, $\mathrm{j}++$, aux $=$ aux $*((z[j, r][[1]]-\operatorname{lz}[j, r][[2]])-(z s[[1]]-I \mathrm{zs}[[2]]))$

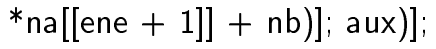

(* Fourth section of Witt's polynomial *)

nd $=$ Expand[Sum[ml[i, r $]^{*}$ (aux $=1 ;$ For $[j=1, j<=$ ene, $\mathrm{j}++$, If $[\mathrm{j} !=\mathrm{i}$, aux $=$ aux* $(((z[j, r][[1]]-I z[j, r][[2]])-(z s[[1]]-I z s[[2]]))$

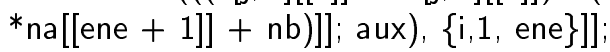

(* Fifth section of Witt's polynomial *)

ne $=$ Expand[na[[ene +1$]]^{*}$ nd];

(* Free up some memory ${ }^{*}$ )

ClearAll[na, nb, nd]; Share[];

(* Witt's Polynomial *)

poly[z_, s_] := Expand[nc + ne] $/ .\{w->z, r->s\}$;

(* Real and imaginary parts of the lens equation to test Witt's solutions *) equis[x_, $\left.y_{-}, s_{-}\right]:=x-\operatorname{Sum}[m \mid[k, s](x-z[k, s][[1]]) /((x-z[k, s][[1]]) \wedge 2$ $+(\bar{y}-z[k, \bar{s}][[2]]) \wedge 2),\{k$, ene $\}] ;$

$y e\left[x_{-}, y_{-}, s_{-}\right]:=y-\operatorname{Sum}[m \mid[k, s](y-z[k, s][[2]]) /((x-z[k, s][[1]]) \wedge 2$

$\left.+(y-z[k, s][[2]])^{\wedge} 2\right),\{k$, ene $\left.\}\right]$

(* Magnification function for images *)

$\operatorname{mag}\left[i m g \_, s_{2}\right]:=1 /(1-(\operatorname{Abs}[\operatorname{Sum}[\mathrm{ml}[\mathrm{k}, \mathrm{s}] /((\mathrm{img}[[1]]+\mid * i m g[[2]])$

$\left.-\left(z[k, s][[1]]+I^{*} z[k, s][[2]]\right)\right)^{\wedge} 2,\{k, 1$, ene $\left.\left.\left.\left.\}\right]\right]\right)^{\wedge} 2\right)$;

(* Parity function for images *)

par[img_, s_] := Sign[mag[img, s]];

$(*$ Equation for the critical curves *)

$\mathrm{p}\left[\mathrm{z}_{-}\right.$, theta_, $\left.\mathrm{s}_{-}\right]:=\operatorname{Product}\left[(\mathrm{z}-(\mathrm{z}[\mathrm{j}, \mathrm{s}][[1]]+\mathrm{I}[\mathrm{zj}, \mathrm{s}][[2]]))^{\wedge} 2,\{\mathrm{j}\right.$, ene $\left.\}\right]$

- Exp[l theta] (aux =0; For $[k=1, k<=$ ene, $k++$, otto $=1$;

For $[j=1, j<=$ ene, $j++$, If $[j !=k$, otto $=$ otto

$\left.\left.*(z-(z[j, s][[1]]+\mid z[j, s][[2]]))^{\wedge} 2\right]\right] ;$ aux = aux + ml[k, s] otto $] ;$ aux $)$;

$(*$ Main loop *)

(* Clear all variables and initialize parameters *)

ClearAll[graph, centerplus, centerminus, aux, witt, critical, zap, amp,

mmax, mmin, caustic, images, ampmax, sspace, scaledamp, scap, slow];

graph $=\{\}$

amp $=\{\} ;$

$\operatorname{ampmax}=0$;

images $=\{\}$;

$\operatorname{mmax}=0$;

$\operatorname{mmin}=5$ ene -5 ;

sspace $=\{\}$;

scaledamp $=\{\}$;

slow $=0 / 10$;

scap $=8 / 10$; 
(* Control to exit when number of images goes below maximum *)

zap = True;

(* Search loop for s between slow and scap *)

For $[s=$ slow, $s<=$ scap \&\& zap, $s+=1 / 100$,

(* Numerical expression for Witt's polynomial *)

aux $=\mathrm{N}[$ poly[w, s], 200];

(* Find all the solutions of the polynomial *)

witt $=$ Solve[aux $==0, \mathrm{w}]$;

(* Reorganize the solutions from complex numbers to ordered pairs *)

aux $=$ Table[w /. Extract[witt, i], $\{i, 1$, Length[witt] $\}]$;

witt $=$ Table[ $\{\operatorname{Re}[$ aux $[[i]]], \operatorname{Im}[$ aux $[[i]]]\},\{i, 1$, Length[aux] $\}]$;

(* Auxiliary variable to sort the solutions $*$ )

centerplus $=$ witt;

(* Remove solutions at the position of a lensing mass that generate divergences $*$ )

For $[\mathrm{k}=1, \mathrm{k}<=$ ene, $\mathrm{k}++$, For[ $\mathrm{j}=1, \mathrm{j}<=$ Length[centerplus], $\mathrm{j}++$,

If $[(($ centerplus[[j]][[1]] - z[k, s][[1]])^2 + (centerplus[[j]][[2]]

$\left.-\mathrm{z}[\mathrm{k}, \mathrm{s}][[2]])^{\wedge} 2\right)==0$, centerplus $=$ Drop[centerplus, $\left.\left.\left.\left.\{\mathrm{j}, \mathrm{j}\}\right] ; j-\right]\right]\right] ;$

(* Remove duplicate solutions *)

For[ $j=1, j<=$ Length[centerplus], $j++$, For[ $k=j+1, k<=$ Length[centerplus],

$\mathrm{k}++$, If $[$ centerplus $[[j]]==$ centerplus[[k]], centerplus $=$

Drop[centerplus, $\{k, k\}] ; k-]]]$;

(* Remove solutions that do not satisfy the lens equation *)

For[j $=1, j<=$ Length[centerplus], $\mathrm{j}++$, If[Chop[equis[centerplus[[j]][[1]],

centerplus[[j]][[2]], s] - zs[[1]], 10^-20] !=0 $\|$ Chop[ye[centerplus[[j]][[1]],

centerplus[[j]][[2]], s] - zs[[2]], 10^-20]!=0, centerplus $=$

Drop[centerplus, $\{\mathrm{j}, \mathrm{j}\}] ; \mathrm{j}-]]$;

(* Make a list of the number of images as a function of $s *$ )

images $=$ Append[images, $\{\mathrm{s}$, Length[centerplus]\}];

(* Exit condition if producing less than the maximum number of images *)

(* When not searching for maximal lensing, remove and make light curves *)

zap $=$ zap \&\& (Length[centerplus] $==5$ ene -5$)$;

( Test for maximum and minimum number of images found $*$ )

If[Length[centerplus] $>$ mmax, $\operatorname{mmax}=$ Length[centerplus]];

If[Length[centerplus] $<\mathrm{mmin}, \mathrm{mmin}=$ Length[centerplus]];

$(*$ Test for the largest amplification factor *)

If[Sum[Abs[mag[centerplus[[i]], s]], \{i, Length[centerplus]\}] > ampmax,

ampmax $=\mathrm{N}[$ Round[Sum[Abs[mag[centerplus[[i]], s]], \{i, Length[centerplus]\}]]]];

(* Calculate the total amplification for all the images present $*$ )

amp $=$ Append[amp, $\{\mathrm{s}$, Sum[Abs[mag[centerplus[[i]], s]], \{i, Length[centerplus]\}]\}];

centerminus $=\{\}$;

(* Separate images of positive and negative magnification *)

For[j $=1, j<=$ Length[centerplus], $j++$, If[par[centerplus[[j]], s] $==-1$,

centerminus $=$ Append [centerminus, centerplus[[j]]]; centerplus $=$

Drop[centerplus, $\{j, j\}] ; j-]]$;

aux $=\{\}$;

critical $=\{\}$;

(* Calculate the critical curves *)

For[phi $=0$, phi $<2 \mathrm{Pi}$, phi $+=2 \mathrm{Pi} / 10$, aux $=$ Solve[N[p[z, phi, s], 10] $==0, \mathrm{z}]$;

For $[\mathrm{j}=1, \mathrm{j}<=$ Length[aux], $\mathrm{j}++$, critical = Append[critical,

$\{\operatorname{Re}[z] /$. aux[[j]], $\operatorname{Im}[z] /$. aux[[j]]\}]]];

caustic $=\{\}$

(* Calculate the caustics *)

For[j $=1, \mathrm{j}<=$ Length[critical], $\mathrm{j}++$, caustic $=$ Append[caustic,

\{equis[critical[[j]]][1]], critical[[j]][[2]], s], ye[critical[[j]][[1]], 
critical[[j]][[2]], s]\}]];

(* Graph images, lenses, source, witt's images and caustic and critical curves *)

graph $=$ Append[graph, MultipleListPlot[centerplus, centerminus, lentes[s], \{zs\}, critical, witt, caustic, SymbolShape $->$ \{PlotSymbol[Star, 5, Filled $->$ False],

PlotSymbol[Star, 5, Filled $->$ False], PlotSymbol[Box, 3, Filled $->$ False],

PlotSymbol[Diamond, 3], PlotSymbol[Box, 0.5], PlotSymbol[Diamond, 2],

PlotSymbol[Box, 0.5]\}, SymbolLabel $->\{$ None, None, \{SetPrecision[s, 2], None,

None, None, None, None, None\}, None, None, None, None\},

SymbolStyle -> \{RGBColor[0, 0, 1], RGBColor[1, 0, 0], RGBColor[0, 0.5, 0.5],

RGBColor[1, 0, 1], RGBColor[1, 0, 0.5], RGBColor[0, 0, 0],

RGBColor[0, 0.5, 0.5]\}, AspectRatio -> Automatic, ImageSize $->85 * 10$,

PlotRange $->\{\{-0.5,0.5\},\{-0.5,0.5\}\}]]$;

(* Clear parameters for next iteration *)

ClearAll[aux, centerplus, centerminus, witt, critical, caustic]];

(* Return max and min number of images and max amplification *)

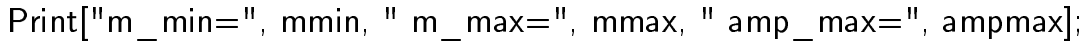

scaledamp $=\{\}$

( $*$ Scale the magnification to plot alongside the number of images *)

scaledamp $=$ Table $[\{\operatorname{amp}[[i]][[1]]$, amp $[[i]][[2]] *(5$ ene -5$) / \operatorname{ampmax}+($ ene +1$) / 2\}$,

$\{\mathrm{i}, 1$, Length[amp]\}];

(* Graph magnification and number of images as a function of $\mathrm{s}^{*}$ )

(* Graph a light curve with maximal lensing signature *)

MultipleListPlot[images, scaledamp, PlotJoined $->\{$ True, True $\}$,

PlotStyle $->\{$ RGBColor $[1,0,0], \mathrm{RGBColor}[0,0,1]\}$, ImageSize $->85 * 10$,

PlotRange $->\{\{$ slow, scap $\},\{0$, Round $[(5$ ene -5$)+($ ene +1$) / 2]\}\}$,

SymbolShape $->\{$ PlotSymbol[Box, 1], PlotSymbol[Box, 1]\},

SymbolStyle $->\{$ RGBColor $[1,0,0]$, RGBColor $[0,0,1]\}]$;

sspace $=\{\}$;

$(*$ Extract values for the angle perturbation that given maximal lensing *)

For $[i=1, i<=$ Length[images], i++, If[images[[i]][[2]] $==(5$ ene -5$)$,

sspace $=$ Append[sspace, images[[i]][[1]]]]];

(* Return the values of the angles allowed for maximal lensing *)

Print[sspace/100];

\section{B.3 Searching for Asymmetric Configurations}

In this section we present the code used to search for asymmetric maximal lensing configurations of point-mass lenses. This search was motivated by results found in Rhie (2001) for the case of $n=4$ identical point masses. We found a range of asymmetric maximal lensing configurations for $n=4$ and $n=5$ identical point masses. For the case of $n=6$ point masses, 21 images was the largest number of images found. This result is 4 images short of the known maximum. Given the resolution with which the search was carried out we conclude that an asymmetric maximal lensing configuration is less unstable and 
therefore less likely to be observed than the symmetric case with a small mass at the center.

The code listed below details a search for maximal lensing in the case of $n=6$ identical point masses located along the lines of symmetry of a regular hexagon. The symmetry is broken by varying the distances from the lenses to the center of the configuration. The masses are paired so that the lens at $0^{\circ}$ and the lens at $180^{\circ}$ maintain the same distance to the origin. Similarly, the lens at $60^{\circ}$ is paired with the lens at $-60^{\circ}$ and the lens at $120^{\circ}$ is paired with the lens at $-120^{\circ}$. Different groupings of the lenses were used in various search schemes. However, completely independent distances for all lenses lead to a representation in terms of Witt's polynomial (2.18) that cannot be computed due to memory limitations. Important differences between this code and the one listed in Sections B.1 and B.2 are highlighted in bold typeface.

( Load package for graphing multiple functions at a time *)

$<<$ Graphics'MultipleListPlot'

$(*$ The value for $\mathrm{n}$ masses in the lens configuration *)

ene $=6$;

(* The $\mathrm{n}$ masses located along the lines of the $\mathrm{n}$ - sided polygon $*$ )

a[i_] $:=\left\{\operatorname{Cos}\left[2 * \mathrm{Pi}^{*}(\mathrm{i}-1) / \mathrm{ene}\right], \operatorname{Sin}\left[2 * \mathrm{Pi}^{*}(\mathrm{i}-1) /\right.\right.$ ene $\left.]\right\}$;

(* Search for the position that leads to maximal lensing *)

(* Pairing of lenses $z 1\left(0^{\circ}\right)-z 3\left(180^{\circ}\right), z 2\left(60^{\circ}\right)-z 6\left(-60^{\circ}\right)$ and $\left.z 3\left(120^{\circ}\right)-z 5\left(-120^{\circ}\right) *\right)$

$\mathrm{z}\left[1, \mathrm{~s}_{-}\right]:=\mathrm{s} * \mathrm{a}[1] / 100$;

$\mathrm{z}\left[2, \mathrm{t}_{-}\right]:=\mathrm{t} * \mathrm{a}[2] / 100$

$z\left[3, u_{-}^{-}\right]:=u^{*} a[3] / 100$;

$\mathrm{z}\left[4, \mathrm{~s}_{-}\right]:=\mathrm{s} * \mathrm{a}[4] / 100$;

$\mathrm{z}\left[5, \mathrm{u}_{-}\right]:=\mathrm{u}^{*} \mathrm{a}[5] / 100$;

$\mathrm{z}\left[6, \mathrm{t}_{-}\right]:=\mathrm{t}^{*} \mathrm{a}[6] / 100$;

(* The $\mathrm{n}$ masses with a total of $1^{*}$ )

For $\left[i=1, i<=\right.$ ene, $\mathrm{i}++, \mathrm{ml}\left[\mathrm{i}, \mathrm{s}_{-}\right]:=1$ /ene];

(* Position of the source *)

zs : $=\{0,0\}$;

(* Array with the location of all the lenses *)

lentes[s_, $\left.t_{-}, u_{-}\right]:=\{z[1, s], z[2, t], z[3, u], z[4, s], z[5, u], z[6, t]\}$;

(* First section of Witt's polynomial *)

na $=$ Expand[Table[(aux $=1 ;$ For[ $k=1, k<=$ ene, $k++$, If $[k !=j$, aux $=$ aux $*($ If $[k==1 \| k==4,(z[k, p][[1]]+\mid z[k, p][[2]]-w)$, If $[k==2 \| k==6,(z[k, q][[1]]+$ I $z[k, q][[2]]-w)$, 
$(z[k, r][[1]]+I z[k, r][[2]]-w)]])]] ;$ aux $),\{j, 1$, ene +1$\}]] ;$

(* Second section of Witt's polynomial *)

$\mathrm{nb}=\operatorname{Expand}\left[\right.$ Sum[ml[i, r] ${ }^{*}$ na[[i]], $\{i, 1$, ene $\left.\left.\}\right]\right] ;$

(* Third section of Witt's polynomial *)

$\mathrm{nc}=\operatorname{Expand}[(\mathrm{w}-(\mathrm{zs}[[1]]+\mathrm{I} z \mathrm{zs}[2]])) *($ aux $=1 ;$ For $[j=1, \mathrm{j}<=$ ene, $\mathrm{j}++$,

aux $=\operatorname{aux} *(($ If $[j==1|| j==4,(z[j, p][[1]]-\mid z[j, p][[2]])$,

If $[j==2 \| j==6,(z[j, q][[1]]-\mid z[j, q][[2]])$,

$(z[j, r][[1]]-\mid z[j, r][[2]])]])-(z s[[1]]-\mid z s[[2]]))$

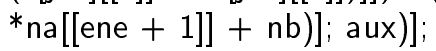

(* Fourth section of Witt's polynomial *)

nd $=$ Expand[Sum[m|[i, r]*(aux $=1$; For $[j=1, j<=$ ene, $j++$, If $[j !=i$,

aux $=\operatorname{aux} *(($ If $[j==1 \| j==4,(z[j, p][[1]]-\mid z[j, p][[2]])$,

If $[j==2 \| j==6,(z[j, q][[1]]-\mid z[j, q][[2]])$,

$(z[j, r][[1]]-\mid z[j, r][[2]])]])-(z s[[1]]-\mid z s[[2]]))$

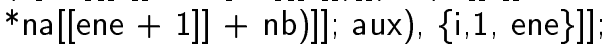

(* Fifth section of Witt's polynomial *)

ne $=\operatorname{Expand}[$ na[[ene +1$]]^{*}$ nd];

(* Witt's Polynomial *)

poly[z_, s_, t_, u_] := Expand $[n c+n e] / .\{w->z, p->s, q->t, r->u\}$;

(*Real and imaginary parts of the lens equation to test Witt's solutions*)

equis[x_, $\left.y_{-}, s_{-}, t_{-}, u_{-}\right]:=x-\operatorname{Sum}[\mathrm{ml}[k, s](x-\operatorname{If}[k==1 \| k==4, z[k, s][[1]]$,

If $[k==2 \| k==6, z[k, t][[1]], z[k, u][[1]]]]) /((x-\operatorname{If}[k==1 \| k==4$,

$z[k, s][[1]], \operatorname{If}[k==2 \| k==6, z[k, t][[1]], z[k, u][[1]]]]) \wedge 2$

$+(y-\operatorname{If}[k==1 \| k==4, z[k, s][[2]], \operatorname{If}[k==2 \| k==6, z[k, t][[2]]$,

$z[k, u][[2]]]]) \wedge 2),\{k$, ene $\}] ;$

ye[x_, y_, s_, t_, u_] $:=y-\operatorname{Sum}[\mathrm{m} \mid[k, \mathrm{~s}](\mathrm{y}-\mathrm{If}[\mathrm{k}==1 \| \mathrm{k}==4, \mathrm{z}[\mathrm{k}, \mathrm{s}][[2]]$,

If $[k==\overline{2} \| \bar{k}==6, z[k, t][[2]], z[k, u][[2]]]]) /((x-\operatorname{If}[k==1 \| k==4$,

$z[k, s][[1]], \operatorname{If}[k==2 \| k==6, z[k, t][[1]], z[k, u][[1]]]]) \wedge 2$

$+(y-I f[k==1 \| k==4, z[k, s][[2]]$, If $[k==2 \| k==6, z[k, t][[2]]$,

$\left.z[k, u][[2]]]])^{\wedge} 2\right),\{k$, ene $\left.\}\right]$;

$(*$ Main loop *)

$(*$ Clear all variables and initialize parameters $*$ )

ClearAll[solutions, aux, witt, mmax, mmin, images,

sspace, scap, slow, tcap, tlow, ucap, ulow];

images $=\{\}$;

$\operatorname{mmax}=0$;

$\operatorname{mmin}=5$ ene -5 ;

sspace $=\{\}$;

slow $=60$;

scap $=70$;

tlow $=30$;

tcap $=40$;

ulow $=80$;

ucap $=90$;

(* Search loop for $s, t$ and $u *$ )

For $[s=$ slow, $s<=$ scap, $s+=1$,

For $[\mathbf{t}=$ tlow, $\mathbf{t}<=$ tcap, $\mathbf{t}+=1$,

For $[u=$ ulow, $\mathbf{u}<=$ ucap, $\mathbf{u}+=1$, 


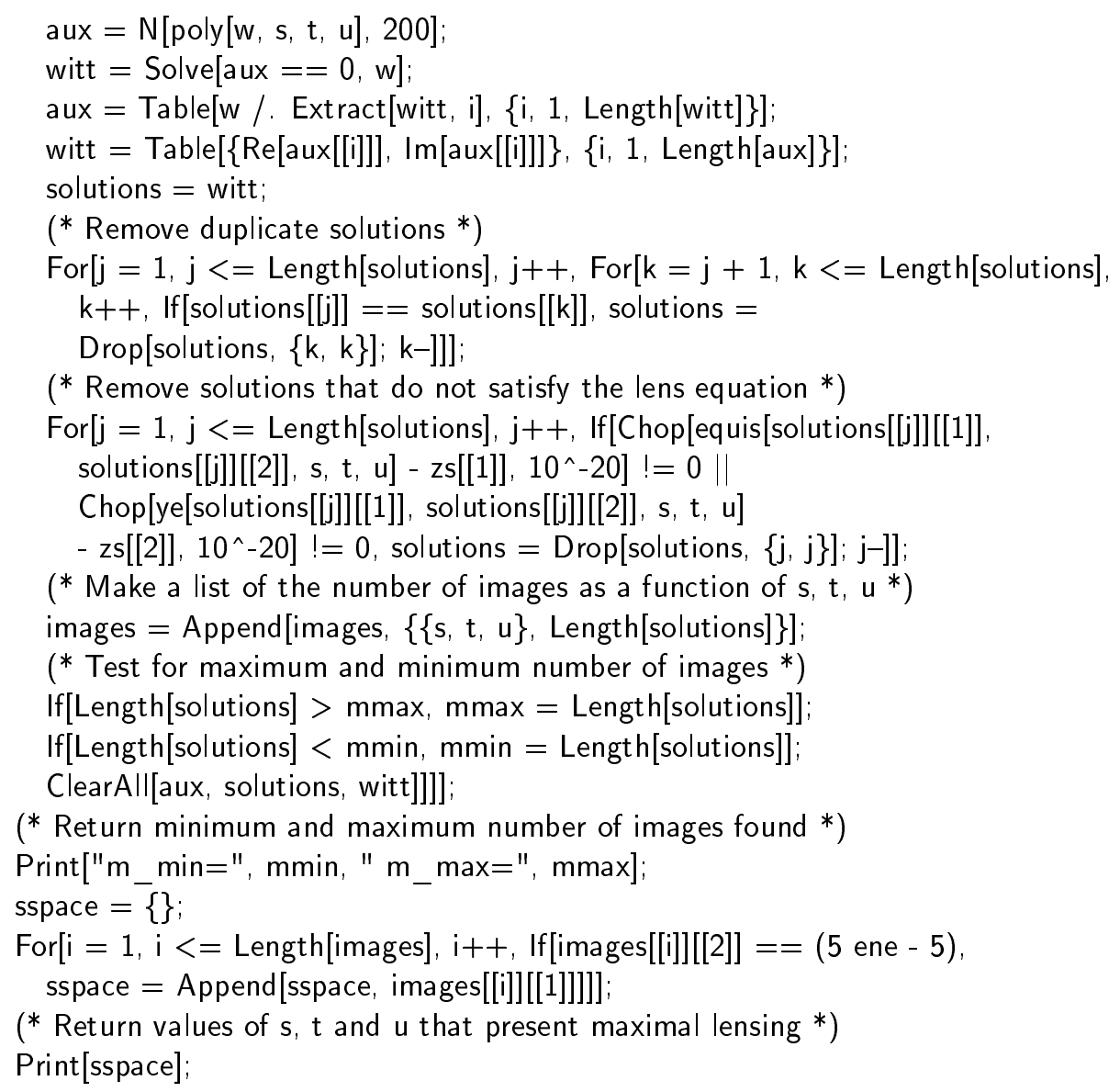

\section{B.4 Perturbations on Asymmetric Configurations}

To study the stability of the asymmetric maximal lensing configurations described in Section 5.3 and found through the numerical simulations described in Section B.3 we set up numerical simulations perturbing the positions of source and lenses as well as perturbations on the mass of the lenses. The results of these simulations can be found in Section 5.4 and the code used for these perturbations is presented in this section. This code is similar to the one used for the perturbations on the symmetric configurations in Section B.2.

The sample code listed below is for the case of $n=5$ identical point-mass lenses located at positions given in polar coordinates by $z_{1}=\left(0.50,0^{\circ}\right), z_{2}=\left(0.90,72^{\circ}\right)$, $z_{3}=\left(0.53,144^{\circ}\right), z_{4}=\left(0.53,-144^{\circ}\right)$ and $z_{5}=\left(0.90,-72^{\circ}\right)$. The radial position of 
one the lens at $z_{2}$ is perturbed in order to find the region of stability that presents maximal lensing. Important differences between this code and the one listed in Section B.2 are highlighted in bold typeface.

(* Load package for graphing multiple functions at a time *)

$<<$ Graphics'MultipleListPlot'

$(*$ This defines the value for $\mathrm{n}$ masses in the lens configuration $*$ )

ene $=5$;

(* The $\mathrm{n}$ masses located along the lines of the $\mathrm{n}$ - sided polygon *)

$\mathrm{a}\left[\mathrm{i}_{-}\right]:=\left\{\operatorname{Cos}\left[2 * \mathrm{Pi}^{*}(\mathrm{i}-1) / \mathrm{ene}\right], \operatorname{Sin}\left[2 * \mathrm{Pi}^{*}(\mathrm{i}-1) / \mathrm{ene}\right]\right\}$;

(* Position of the lenses at $0.50,0.90,0.53,0.53$ and 0.90 from the center ${ }^{*}$ ) z[1, s_] $:=50 * a[1] / 100$;

(* Parameter $s$ to control the perturbation in the radial position of $\mathrm{z2} *$ )

$\mathrm{z}\left[2, \mathrm{~s}_{-}\right]:=\mathrm{s} * \mathrm{a}[2] / 100$

$z\left[3, s_{-}\right]:=53 * a[3] / 100$;

$z\left[4, s_{-}\right]:=53 * a[4] / 100$;

$z\left[5, s_{-}\right]:=90 * a[5] / 100$;

(* The $\mathrm{n}$ masses with a normalized total of $1 *$ )

$\mathrm{ml}\left[\mathrm{i}_{-}, \mathrm{s}_{-}\right]:=1$ ene;

(* Position of the source *)

zs $:=\{0,0\}$;

(* Array with the location of all the lenses *)

lentes[s_] := Table[z[i, s], $\{\mathrm{i}$, ene $\}]$;

(* First section of Witt's polynomial *)

na $=$ Expand[Table[(aux = 1; For $[k=1, k<=$ ene, $k++$, If $[k !=j$,

aux $=\operatorname{aux} *(z[k, r][[1]]+\mid z[k, r][[2]]-w)]] ;$ aux $),\{j, 1$, ene +1$\}]] ;$

(* Second section of Witt's polynomial *)

$\mathrm{nb}=\operatorname{Expand}[$ Sum[ml[i, r]*na[[i]], $\{i, 1$, ene $\}]] ;$

(* Third section of Witt's polynomial *)

$n c=$ Expand $[(w-(z s[[1]]+\mid z s[[2]])) *(a u x=1 ;$ For $[j=1, j<=$ ene, $j++$, aux $=$ aux* $(((z[j, r][[1]]-I z[j, r][[2]])-(z s[[1]]-I z s[[2]]))$

$*_{\text {na[ }}[$ ene +1$\left.\left.\left.]\right]+\mathrm{nb}\right)\right]$; aux $\left.)\right]$;

(* Fourth section of Witt's polynomial *)

nd $=$ Expand[Sum $\left[\mathrm{ml}[\mathrm{i}, \mathrm{r}]^{*}\right.$ (aux $=1$; For $[\mathrm{j}=1, \mathrm{j}<=$ ene, $\mathrm{j}++$, If $[\mathrm{j} !=\mathrm{i}$, aux $=$ aux* $(((z[j, r][[1]]-\mathrm{I} z[\mathrm{j}, \mathrm{r}][[2]])-(\mathrm{zs}[[1]]-\mathrm{I} z \mathrm{~s}[[2]]))$

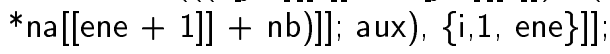

$(*$ Fifth section of Witt's polynomial *)

ne $=$ Expand[na[[ene +1$]]^{*}$ nd];

$\left(*\right.$ Free up some memory $\left.{ }^{*}\right)$

ClearAll[na, nb, nd]; Share[];

(* Witt's Polynomial *)

poly[z_, s_] := Expand[nc $+n e] / .\{w->z, r->s\}$;

(* Real and imaginary parts of the lens equation to test Witt's solutions *)

equis[x_, y_, s_] := x - Sum[m|[k, s] $(x-z[k, s][[1]]) /((x-z[k, s][[1]]) \wedge 2$

$+(y-z[k, \bar{s}][[2]]) \wedge 2),\{k$, ene $\}] ;$

ye[x_, y_, s_] :=y - Sum $\left[m \mid[k, s](y-z[k, s][[2]]) /\left((x-z[k, s][[1]])^{\wedge} 2\right.\right.$ 
$\left.+(y-z[k, s][[2]])^{\wedge} 2\right),\{k$, ene $\left.\}\right]$

(* Magnification function for images *)

$\operatorname{mag}\left[i m g \_, s_{-}\right]:=1 /(1-(\operatorname{Abs}[\operatorname{Sum}[\mathrm{ml} \mid \mathrm{k}, \mathrm{s}] /((\mathrm{img}[[1]]+\mid * i m g[[2]])$

$\left.-\left(z[k, s][[1]]+I^{*} z[k, s][[2]]\right)\right)^{\wedge} 2,\{k, 1$, ene $\left.\left.\left.\left.\}\right]\right]\right)^{\wedge} 2\right)$;

(* Parity function for images $*$ )

par[img_, s_] := Sign[mag[img, s]];

$(*$ Equation for the critical curves *)

$\mathrm{p}\left[\mathrm{z}_{-}\right.$, theta_, $\left.\mathrm{s}_{-}\right]:=\operatorname{Product}\left[(\mathrm{z}-(\mathrm{z}[\mathrm{j}, \mathrm{s}][[1]]+\mathrm{I} z[\mathrm{j}, \mathrm{s}][[2]]))^{\wedge} 2,\{\mathrm{j}\right.$, ene $\left.\}\right]$

- Exp[l theta] (aux =0; For $[k=1, k<=$ ene, $k++$, otto $=1$;

For $[j=1, j<=$ ene, $j++$, If $[j !=k$, otto $=$ otto

$\left.\left.*(z-(z[j, s][[1]]+\mid z[j, s][[2]]))^{\wedge} 2\right]\right] ;$ aux = aux + ml[k, s] otto $] ;$ aux $)$;

$(*$ Main loop *)

(* Clear all variables and initialize parameters $*$ )

ClearAll[graph, centerplus, centerminus, aux, witt, critical, zap, amp,

mmax, mmin, caustic, images, ampmax, sspace, scaledamp, scap, slow];

graph $=\{\}$

amp $=\{\}$

$\operatorname{ampmax}=0$;

images $=\{\}$

$\operatorname{mmax}=0$;

$\operatorname{mmin}=5$ ene -5 ;

sspace $=\{\}$;

scaledamp $=\{\}$

slow $=899 / 10$

scap $=901 / 10$;

(* Control to exit when number of images goes below maximum *)

zap = True;

(* Search loop for s between slow and scap *)

For $[\mathrm{s}=$ slow, $\mathrm{s}<=$ scap \&\& zap, $\mathrm{s}+=1 / 100$,

(* Numerical expression for Witt's polynomial *)

aux $=\mathrm{N}[$ poly[w, s], 200];

(* Find all the solutions of the polynomial *)

witt $=$ Solve $[\mathrm{aux}==0, \mathrm{w}]$;

(* Reorganize the solutions from complex numbers to ordered pairs *)

aux $=$ Table[w /. Extract[witt, i], $\{i, 1$, Length[witt] $\}]$;

witt $=$ Table[\{Re[aux[[i]]], Im[aux[[i]]]\}, \{i, 1, Length[aux]\}];

(* Auxiliary variable to sort the solutions *)

centerplus $=$ witt;

(* Remove solutions at the position of a lensing mass that generate divergences $*$ )

For[ $k=1, k<=$ ene, $\mathrm{k}++$, For[ $\mathrm{j}=1, \mathrm{j}<=$ Length[centerplus], $\mathrm{j}++$,

If $[(($ centerplus[[j]][[1]] - z[k, s][[1]])^2 + (centerplus[[j]][[2]]

$\left.-\mathrm{z}[\mathrm{k}, \mathrm{s}][[2]])^{\wedge} 2\right)==0$, centerplus $=$ Drop[centerplus, $\left.\left.\left.\left.\{\mathrm{j}, \mathrm{j}\}\right] ; j-\right]\right]\right] ;$

(* Remove duplicate solutions *)

For[ $j=1, j<=$ Length[centerplus], $j++$, For[ $k=j+1, k<=$ Length[centerplus], $\mathrm{k}++$, If $[$ centerplus $[[j]]==$ centerplus[[k]], centerplus $=$

Drop[centerplus, $\{k, k\}] ; k-]]]$;

(* Remove solutions that do not satisfy the lens equation *)

For[ $=1, \mathrm{j}<=$ Length[centerplus], $\mathrm{j}++$, If[Chop[equis[centerplus[[j]][[1]], centerplus[[j]][[2]], s] - zs[[1]], 10^-20] !=0 $\|$ Chop[ye[centerplus[[j]][[1]], centerplus[[j]][[2]], s] - zs[[2]], 10^-20]!=0, centerplus $=$ Drop[centerplus, $\{\mathrm{j}, \mathrm{j}\}]$; $\mathrm{j}-]$ ];

(* Make a list of the number of images as a function of $\mathrm{s} *$ ) 
images $=$ Append[images, $\{\mathrm{s}$, Length[centerplus]\}];

(* Exit condition if producing less than the maximum number of images $*$ )

(* When not searching for maximal lensing, remove and make light curves *)

zap $=$ zap \&\& (Length[centerplus] $==5$ ene -5$)$;

(* Test for maximum and minimum number of images found *)

If[Length[centerplus] $>$ mmax, $\operatorname{mmax}=$ Length[centerplus]];

If[Length[centerplus] $<\mathrm{mmin}, \mathrm{mmin}=$ Length[centerplus]];

(* Test for the largest amplification factor *)

If[Sum[Abs[mag[centerplus[[i]], s]], $\{i$, Length[centerplus]\}] $>$ ampmax,

ampmax $=\mathrm{N}[$ Round[Sum[Abs[mag[centerplus[[i]], s]], $\{\mathrm{i}$, Length[centerplus]\}]]]];

(* Calculate the total amplification for all the images present $*$ )

amp $=$ Append[amp, $\{\mathrm{s}$, Sum[Abs[mag[centerplus[[i]], s]], \{i, Length[centerplus]\}]\}];

centerminus $=\{\}$;

(* Separate images of positive and negative magnification *)

For[j $=1, j<=$ Length[centerplus], j++, If[par[centerplus[[j]], s] == -1 ,

centerminus $=$ Append $[$ centerminus, centerplus[[j]]]; centerplus $=$

Drop[centerplus, $\{j, j\}] ; j-]$;

aux $=\{\}$;

critical $=\{\}$;

$(*$ Calculate the critical curves $*)$

For[phi $=0$, phi $<2 \mathrm{Pi}$, phi $+=2 \mathrm{Pi} / 10$, aux $=$ Solve[N[p[z, phi, s], 10] $==0, \mathrm{z}]$;

For $[\mathrm{j}=1, \mathrm{j}<=$ Length[aux], $\mathrm{j}++$, critical = Append[critical,

$\{\operatorname{Re}[z] /$. aux [[j]], Im[z] /. aux[[j]]\}]]];

caustic $=\{\}$

$(*$ Calculate the caustics $*)$

For[j $=1, \mathrm{j}<=$ Length[critical], $\mathrm{j}++$, caustic $=$ Append[caustic, \{equis[critical[[j]][[1]], critical[[j]][[2]], s], ye[critical[[j]][[1]],

critical[[j]]][2]], s]\}]];

(* Graph images, lenses, source, witt's images and caustic and critical curves *)

graph = Append[graph, MultipleListPlot[centerplus, centerminus, lentes[s], \{zs\},

critical, witt, caustic, SymbolShape $->$ \{PlotSymbol[Star, 5, Filled $->$ False],

PlotSymbol[Star, 5, Filled -> False], PlotSymbol[Box, 3, Filled -> False],

PlotSymbol[Diamond, 3], PlotSymbol[Box, 0.5], PlotSymbol[Diamond, 2],

PlotSymbol[Box, 0.5]\}, SymbolLabel $->\{$ None, None, \{SetPrecision[s, 2], None,

None, None, None, None, None\}, None, None, None, None $\}$,

SymbolStyle -> \{RGBColor[0, 0, 1], RGBColor[1, 0, 0], RGBColor[0, 0.5, 0.5],

RGBColor[1, 0, 1], RGBColor[1, 0, 0.5], RGBColor[0, 0, 0],

RGBColor[0, 0.5, 0.5]\}, AspectRatio -> Automatic, ImageSize $->85 * 10$,

PlotRange $->\{\{-0.5,0.5\},\{-0.5,0.5\}\}]]$;

(* Clear parameters for next iteration *)

ClearAll[aux, centerplus, centerminus, witt, critical, caustic]];

(* Return max and min number of images and max amplification *)

Print ["m_min=", mmin, " m_max=", mmax, " amp_max=", ampmax];

scaledam $\bar{p}=\{\}$

(* Scale the magnification to plot alongside the number of images $*$ )

scaledamp $=$ Table[\{amp[[i]][[1]], amp $[[i]][[2]] *(5$ ene -5$) /$ ampmax $+($ ene +1$) / 2\}$,

$\{i, 1$, Length[amp]\}];

(* Graph magnification and number of images as a function of $s *$ )

(* Graph a light curve with maximal lensing signature *)

MultipleListPlot[images, scaledamp, PlotJoined $->\{$ True, True\},

PlotStyle $->\{$ RGBColor $[1,0,0]$, RGBColor $[0,0,1]\}$, ImageSize $->85^{*} 10$,

PlotRange $->\{\{$ slow, scap $\},\{0$, Round $[(5$ ene -5$)+($ ene +1$) / 2]\}\}$,

SymbolShape $->$ PPlotSymbol[Box, 1], PlotSymbol[Box, 1]\},

SymbolStyle -> RRGBColor[1, 0, 0], RGBColor[0, 0, 1]\}]; 
sspace $=\{\} ;$

(* Extract values for the position perturbation that given maximal lensing *)

For $[i=1, i<=$ Length[images], i++, If[images[[i]][[2]] $==(5$ ene -5$)$,

sspace $=$ Append[sspace, images[[i]][[1]]]]];

(* Return values of maximal lensing positions for $z 2 *$ )

Print[sspace/100]; 